\title{
Adolescent Health Risk Behaviors: An Examination of the Co- occurrence of Risk Behaviors in a National Sample of U.S. High School Adolescents
}

Halima Ahmadi-Montecalvo

Follow this and additional works at: https://researchrepository.wvu.edu/etd

\section{Recommended Citation}

Ahmadi-Montecalvo, Halima, "Adolescent Health Risk Behaviors: An Examination of the Co-occurrence of Risk Behaviors in a National Sample of U.S. High School Adolescents" (2016). Graduate Theses,

Dissertations, and Problem Reports. 5036.

https://researchrepository.wvu.edu/etd/5036

This Dissertation is protected by copyright and/or related rights. It has been brought to you by the The Research Repository @ WVU with permission from the rights-holder(s). You are free to use this Dissertation in any way that is permitted by the copyright and related rights legislation that applies to your use. For other uses you must obtain permission from the rights-holder(s) directly, unless additional rights are indicated by a Creative Commons license in the record and/ or on the work itself. This Dissertation has been accepted for inclusion in WVU Graduate Theses, Dissertations, and Problem Reports collection by an authorized administrator of The Research Repository @ WVU.

For more information, please contact researchrepository@mail.wvu.edu. 
Adolescent Health Risk Behaviors: An Examination of the Co-occurrence of Risk Behaviors in a National Sample of U.S. High School Adolescents

\title{
Halima Ahmadi-Montecalvo, MPH
}

\author{
Dissertation submitted \\ to the School of Public Health \\ at West Virginia University
}

in partial fulfillment of the requirements for the degree of

Doctor of Philosophy in

Social and Behavioral Sciences

Geri Dino, Chair, PhD

Lesley Cottrell, PhD

Christa L. Lilly, PhD

Traci Jarrett, PhD, MPH

Keith Zullig, PhD, MSPH

Department of Social and Behavioral Sciences

\section{Morgantown, West Virginia}

2016

Keywords: adolescents, risk behaviors, problem behavior theory, YRBSS 


\section{ABSTRACT \\ Adolescent Health Risk Behaviors: An Examination of the Co-occurrence of Risk Behaviors in a National Sample of U.S. High School Adolescents}

\section{Halima Ahmadi-Montecalvo}

Introduction: Research on the health status of United States' youths has shown that the main adolescent health threats result from behavioral choices (health risk behaviors) that place boys and girls at risk for leading causes of morbidity and mortality. The aims of this study were to examine the co-occurrence of health risk behaviors and determine patterns of the co-occurrence of risk behaviors that may differ based on sex (aim 1); the association of health risk behaviors with weight status (aim 2); and the association of tobacco use, poor dietary habits, physical inactivity, and sedentary behavior among adolescents using nationally representative data (aim 3).

Methods: This study uses cross-sectional data from the Youth Risk Behavior Surveillance System (YRBSS) conducted between the years 2012 and 2013. The sampling frame for this survey included all adolescents in Grades 9 to 12 who attended regular public, private, or Catholic schools in the 50 states and the District of Columbia. The main outcomes of interest for the first two studies included indicators of health risk behaviors including substance use, intentional and unintentional injuries, risky sexual behavior, mental health, bullying, unhealthy weight control, poor dietary habits, physical inactivity, and sedentary behavior. The main outcome for the third study was tobacco use. Latent class analysis was conducted to determine the co-occurrence of health risk behaviors among adolescents. Regression analyses were conducted to examine the associations between weight status and risky behaviors for the second study; and tobacco use, poor dietary habits, physical inactivity, and sedentary behavior.

Results: With respect to findings for aim one, the latent class analysis yielded five distinct subgroups (classes). The majority of adolescents belonged to the low risk subgroups; however, our analyses identified three distinct high risk subgroups, high risk-depressed and suicidal youth, high risk- tobacco and alcohol users, and high risk-polysubstance users. Girls and boys both reported low risk and high risk behaviors. However, girls had a distinct subgroup with particularly high probabilities of depressive symptoms and suicidal behavior.

The second aim examining the association of weight status and risky behavior found that compared to normal weight students, obese students were more likely to use cigarettes (AOR = $1.44,95 \% \mathrm{CI}, 1.15-1.79)$, less likely to engage in behaviors that result in unintentional injury (e.g. driving drunk, riding with a drunk driver, texting and driving) (AOR $=0.83 ; 95 \% \mathrm{CI}, 0.73$ 0.95), more likely to get bullied on school property, and electronically (AOR $=1.47,95 \% \mathrm{CI}$, $1.28-1.68 ; \mathrm{AOR}=1.20,95 \% \mathrm{CI}, 1.003-1.447$, respectively) and more likely to engage in unhealthy weight control ( $\mathrm{AOR}=7.56,95 \% \mathrm{CI}, 6.33-9.04)$. Findings for the third aim showed that both male and female adolescents who engaged in poor dietary behavior were more likely to use tobacco than those who did not engage in poor dietary behavior. For instance, both boys and girls who skipped breakfast every day for the past seven days were more likely to use tobacco than those who did not skip breakfast $(\mathrm{AOR}=1.54,95 \% \mathrm{CI} 1.16-2.03$ and $\mathrm{AOR}=1.32,95 \% \mathrm{CI}$ 1.06-1.64, respectively). Physical inactivity and playing video or computer games more than three hours per day yielded an inverse association with tobacco use among boys (AOR $=0.62$, 95\% CI 0.49-0.79). 
Conclusions: Distinct subgroups of risky behaviors were identified for the study sample. Differences were found in the pattern of the co-occurrence of risky behavior by sex. Obesity was found to be positively associated with most of the risk behaviors examined in this project. The results of the third aim found that tobacco use was significantly associated with poor dietary habits, physical inactivity, and sedentary behavior. Longitudinal studies of risky behavior among adolescents are needed to establish definitive conclusions and to prove causality. In addition, future research is needed to evaluate risk behaviors among adolescents by sociodemographic factors and geographic region. 


\section{DEDICATION}

I dedicate my doctoral dissertation to my late father, Abdul Raouf Ahmadi. Thank you for providing the foundation from which I continue to grow and to pursue my dreams wholeheartedly. Thank you for your unconditional love, support, and encouragement throughout my life. I am forever grateful to have had you as my dad. I miss you every day. I will always love you.

\section{ACKNOWLEGEMENTS}

I would like to express my heartfelt gratitude to my family. To my late father, Abdul Raouf Ahmadi, who always believed in me, encouraged me, and loved me unconditionally. To my mother, who has provided encouragement, unconditional love, and moral support. To my husband, Jason, who has been my rock and without whom this work would not have been possible. Thank you for your encouragement, support, and your patience throughout my studies. I love you for all the sacrifices you have made. To my daughter Jaelyn, thank you for brining so much joy and happiness into my life.

I owe my deepest gratitude to Dr. Geri Dino who has been an excellent mentor and committee chair and who always has provided me timely feedback and support throughout my studies. Thank you for pushing me to think critically and giving me the freedom to explore my own ideas, and at the same time providing guidance on various problems and crises I have had. Without your support, encouragement, and friendship this dissertation would not have been possible. I would like to convey my gratitude to Dr. Christa Lilly from whom I have gained statistical knowledge and skills that will prove valuable in my career. I truly appreciate your constant support throughout my PhD studies. You are an amazing educator. I would like express my thanks to my committee members Dr. Keith Zullig, Dr. Lesley Cottrell, and Dr. Traci Jarrett for your guidance and encouragement throughout my $\mathrm{PhD}$ studies.

Last but not least, special thanks go to the faculty and staff at the WVU Health Science Center, and the School of Public Health for all your support, and helpful advice throughout my PhD studies. 


\section{TABLE OF CONTENTS}

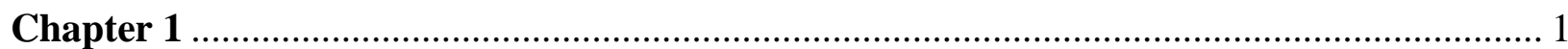

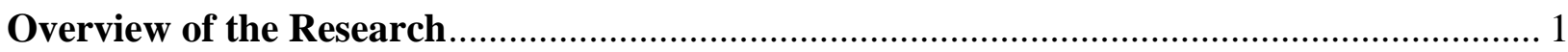

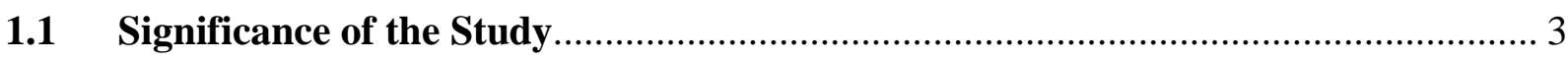

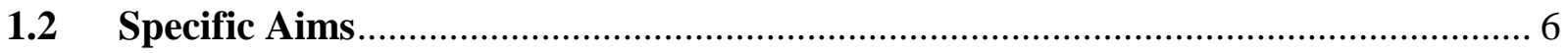

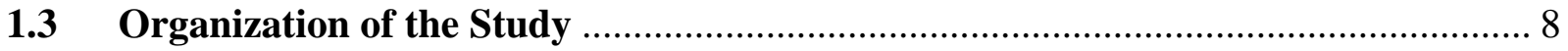

Chapter 2 .

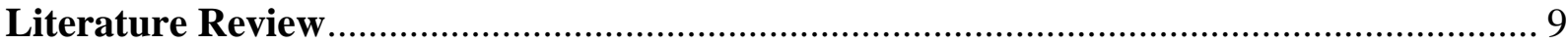

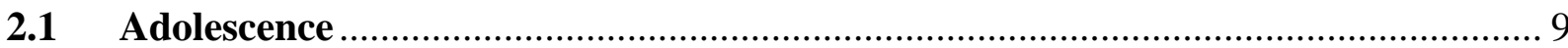

2.2 Overview of Adolescent Risk Behaviors and Factors ………………………........ 10

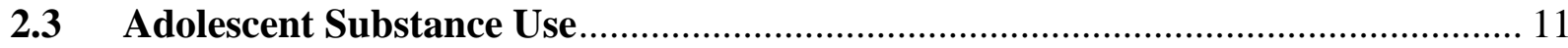

2.4 Behaviors that Contribute to Unintentional Injuries ………………………….... 15

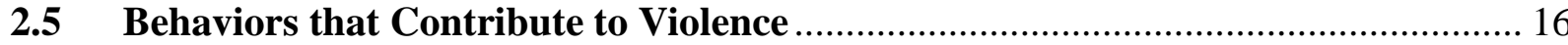

2.6 Adolescent Risky Sexual Behavior ....................................................................... 20

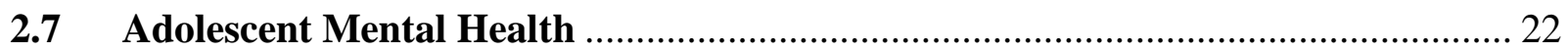

2.8 Adolescent Obesity, Poor Dietary Behaviors, and Sedentary Behaviors ………..... 25

2.9 Theories of Adolescent Risk Behaviors …………………………………………... 26

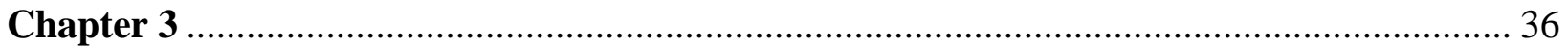

A latent class analysis of the co-occurrence of risk behaviors among adolescents ............... 36

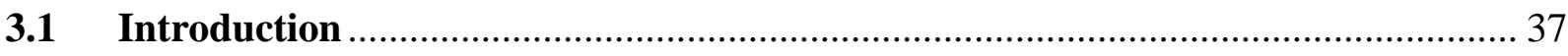

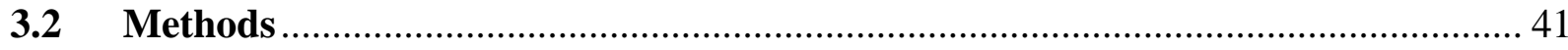

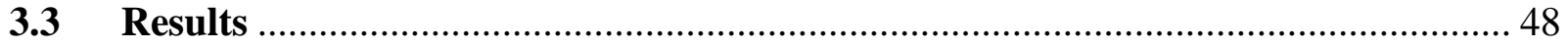

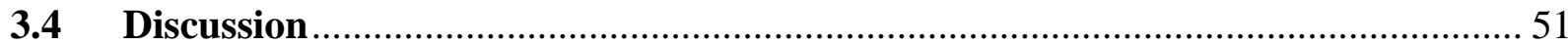

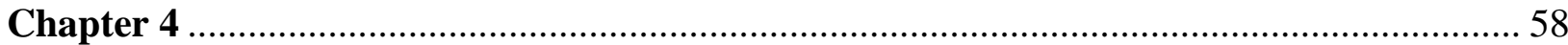

Overweight and obesity and health risk behaviors among high school adolescents in the

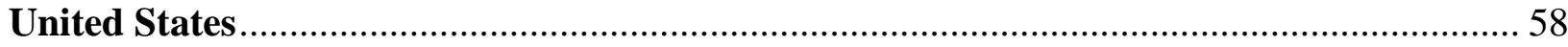

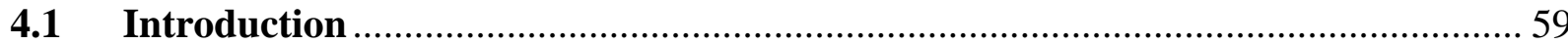

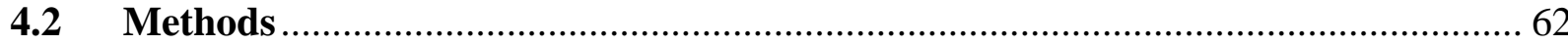

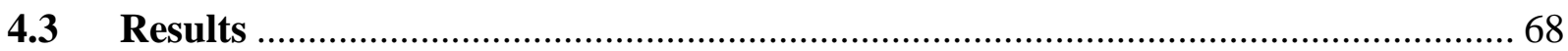

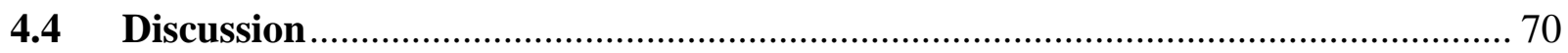


Chapter 5

Factors associated with tobacco use, poor dietary habits, and physical inactivity and sedentary behavior among high school adolescents in the U.S.

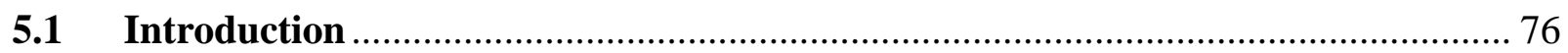

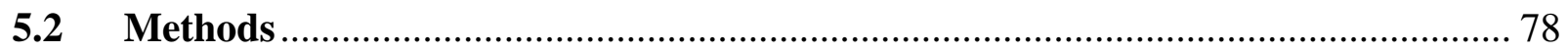

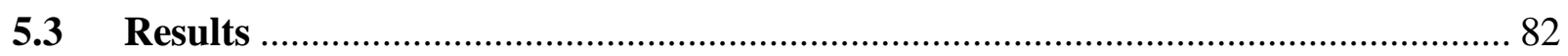

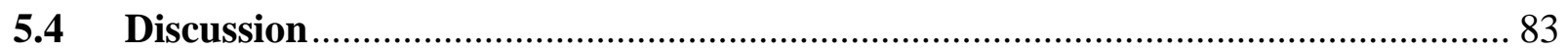

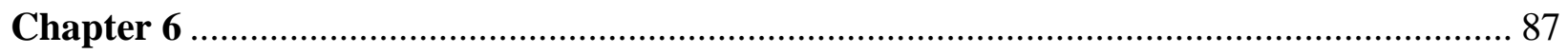

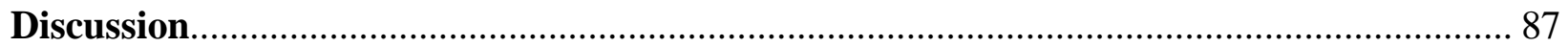

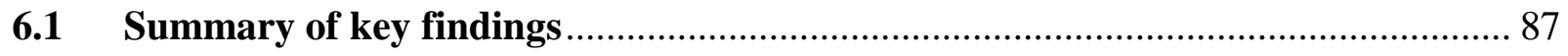

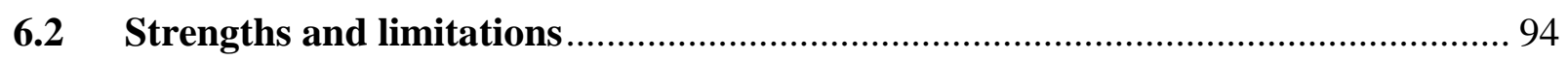

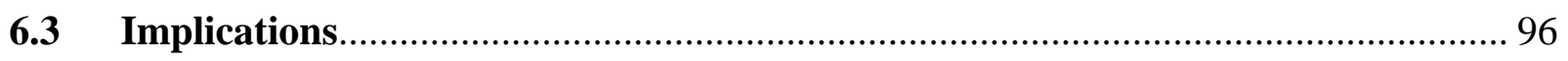

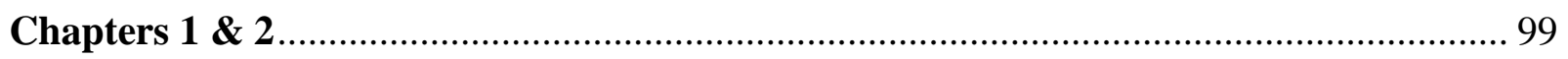

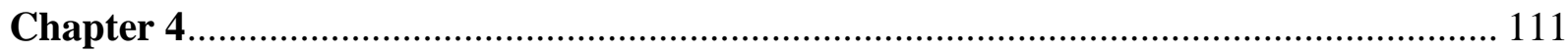

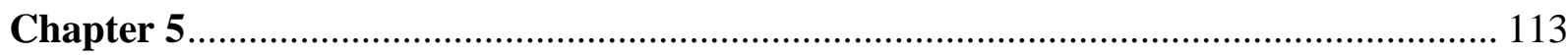

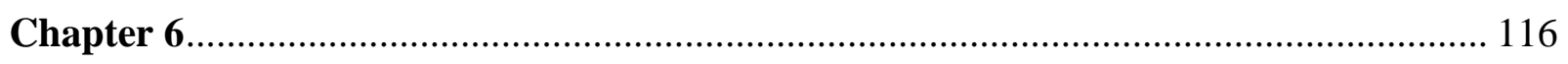

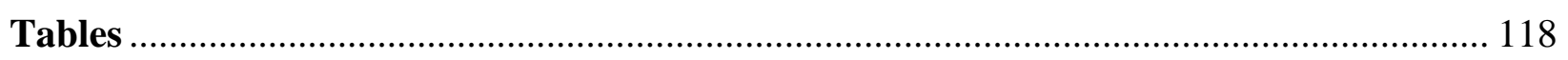

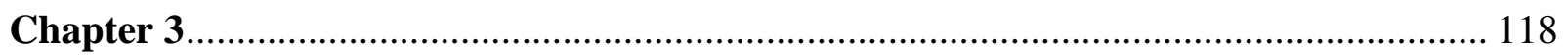

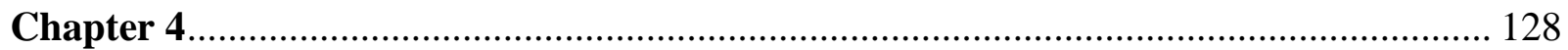

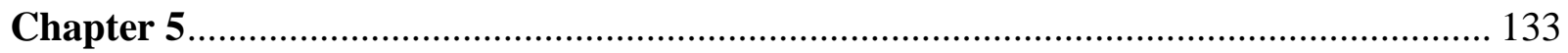




\section{Definition of Terms}

The following terms are consistently used throughout this study; therefore, definitions are provided below. Other terms, which are not frequently used, but require definitions, are explained as they are introduced.

Adolescence refer to young people from age 10-18 years old who are vulnerable to adopting risky behaviors in this transitional stage of development process from childhood to adulthood. Other terms used interchangeably include teens, youth, and teenagers.

Risky Behaviors/Health-Risk Behaviors (HRBs) refer to activities that increase one's likelihood of developing negative health outcomes immediately and at some point in the future. HRBs include substance use including tobacco, alcohol and other illicit drug use, risky sexual behaviors, weapon carrying, poor dietary habits and sedentary lifestyles. Jessor (1991) defines HRBs as "HRBs can jeopardize the accomplishment of normal development of expected social roles, the acquisition of essential skills, the achievement of a sense of adequacy and competence, and the appropriate preparation for transition to the next stage in the life trajectory, young adulthood.’(p. 599)

Health Risk Factors refer to any attribute, characteristic or exposure of an individual that increases the likelihood of developing a disease or injury (i.e., depression). Note: In this project reference to $H R B s$ include depression as a risk factor contributing to risky behaviors.

Youth Risk Behavior Surveillance System (YRBSS) refers to the surveillance system developed by the Centers for Disease Control and Prevention (CDC). YRBSS has been used since 1990 to measure the following six adolescent behavioral risk areas: tobacco use, alcohol, and other drugs use, engagement in risky sexual activities, activities that lead to injury, dietary habits, and physical activity. 


\section{Chapter 1}

\section{Overview of the Research}

Adolescence is when many youth begin to engage in risk-taking behaviors, including behaviors that contribute to unintentional injuries and violence, alcohol, tobacco, and other drug use, sexual behaviors that contribute to unintentional pregnancy and sexually transmitted infections including HIV infection, and poor dietary behaviors and physical inactivity (Centers for Disease Control and Prevention [CDC], 2014). These health risk behaviors (HRBs) contribute to the majority of morbidity and mortality among adolescents and adults in the United States (Kann et al., 2014).

The leading causes of mortality for adolescents include unintentional injuries (48\%) with vehicular injuries accounting for the majority of these deaths, homicide (13\%), and suicide (11\%) (Minino, 2010). Major causes of morbidity among adolescents include substance use (tobacco, alcohol and other drug use), risky sexual behaviors, and obesity resulting from poor dietary behaviors and physical inactivity (Blum and Qureshi, 2011). Nationwide, 35\% of high school students reported current alcohol use ${ }^{1}$ and $22 \%$ of students reported current tobacco use ${ }^{2}$ in the same time-period (Kann et al., 2014). Research shows that $34 \%$ of high school students reported being currently sexually active ${ }^{3}$ and only $60 \%$ reported that either they or their partner had used a condom during last sexual intercourse (CDC, 2014). Adolescents who engage in unprotected sexual activity have a higher risk for sexually-transmitted infections (STIs) including HIV, and unwanted pregnancy. Recent data indicate an estimated 329,772 births;

\footnotetext{
1 Had at least one drink of alcohol on at least one day during the 30 days before the Youth Risk Behavior Surveillance System (YRBSS) survey was administered in 2012

2 Cigarette use, current smokeless tobacco use, or current cigar use at least one day during the 30 days before the YRBSS survey was administered in 2012

3 Engaged in sexual intercourse with at least one person during the 3 months before the survey
} 
548,032 cases of chlamydia, gonorrhea, and syphilis; and 2,240 cases of HIV annually among youth aged 15-19 years (Kann et al., 2014). The negative health consequences of STIs during adolescence include pelvic inflammatory disease, infertility, ectopic pregnancy, preterm births, and fetal abnormalities (Advocates for Youth, 2010; Hamilton, Martin, \& Ventura, 2010). In addition, adolescent unwanted pregnancy may not only compound socioeconomic disadvantages such as lower educational attainment and lower socioeconomic status, but also increase the risk of poor health outcomes for the infant and the mother (Hamilton et al., 2010).

Research also shows that mental health challenges, such as depression experienced during adolescence have adverse effects on overall health and development (Bhatia \& Bhatia, 2007) of youth. Recent prevalence rates on adolescent depression indicate that $30 \%$ of students nationwide had felt depressive symptoms ${ }^{4}$ (Kann et al., 2014). Studies show that one of the strongest indicators of depression is suicidal behavior (ideation, thoughts, or attempts) (Swahn \& Bossarte, 2007). Frequent HRBs in adolescence that have been associated with depression include substance use including alcohol and marijuana use (Bossarte, Simon, \& Swahn, 2008; Groves, Stanley, \& Sher, 2007; Swahn \& Bossarte, 2007), and engaging in risky sexual activity (Brown, Tolou-Shams et al., 2006). Recently among youth, bullying has risen as a primary public health concern. Recent data from the CDC, show that nearly $20 \%$ of adolescents had been bullied on school property and $15 \%$ of adolescents had been electronically bullied during the 12 months before the YRBSS survey (Kann et al., 2014).

Research has indicated a higher level of vulnerability of risky behaviors for overweight/obese adolescents compared to their normal weight peers (Farhat, Iannotti, \& Simons-Morton, 2010) which could lead to experimentation with substance use, and unhealthy

\footnotetext{
${ }^{4} \mathrm{Had}$ felt so sad or hopeless almost every day for two or more consecutive weeks, that they stopped doing some usual activities during the 12 months before the YRBSS survey was administered in 2012
} 
weight control, in addition to depressive symptomology and suicidality. Overweight/Obesity trends, largely driven by poor eating habits and physical inactivity, have increased steadily over the years among adolescents in the US. Nationally, 32\% of 2-19-year-old children are overweight or obese (Ogden et al., 2014). Although complications and adverse health effects of childhood overweight and obesity are well documented and include metabolic health risk, chronic diseases, and psychosocial issues (Ebbeling, Pawlak, \& Ludwig, 2002; Ludwig, 2007), not much is known about the relationship of overweight/obesity with other risky behaviors in adolescents.

\subsection{Significance of the Study}

Research illustrates that adolescent risk behaviors co-occur and that engaging in one risk behavior serves to heighten the risk for engaging in other risky behavior(s) (MacArthur et al., 2012; Weden and Zabin, 2005). Established literature illustrate that HRBs such as substance use (i.e., smoking, drinking, illicit drug use), risky sexual behaviors, and behaviors contributing to violence can co-occur (Hale, Fitzgerald-Yau, \& Viner, 2014; Smith \& Sallee, 2008; Zweig, Lindberg, \& McGinley, 2001; Zweig, Phillips, \& Lindberg, 2002). However, typical adolescent health risk research has addressed specific risks individually (e.g., cigarette smoking, sexual activity, or violence) or a limited number of risks (e.g., substance use and sexual activity; depression and substance use; substance use, depression and violence). This is surprising since as early as the 1970's, Richard Jessor and his colleague (Jessor \& Jessor, 1977) highlighted the importance of the interrelationships between various risky behaviors and factors that contribute to risky behaviors overall. Jessor and Jessor developed a theory called the Problem Behavior Theory (PBT), that addressed the co-occurrence of what they called 'problem behaviors.' 
Problem behavior is defined as "any behavior that departs from the social and legal norms of society and causes a social control response from external sources (Jessor \& Jessor, 1977). PBT conceptualizes that behavior emerges out of the structure and interaction of three systems (Jessor, 1991; Jessor et al., 1995), the behavior system, the personality system, and the perceived environment system. Each of these systems is composed of variables that either serve as instigations for engaging in 'problem behavior' or control against engaging in 'problem behavior' and the balance between these instigations and controls determine the level of tendencies for 'problem behavior' within each system. The behavior system includes problem behaviors such as alcohol use, binge drinking, cigarette smoking, marijuana use, other illicit drug use, risky sexual behaviors, and other deviant/delinquent behaviors are seen as problem behaviors (Jessor, 1991; Jessor et al., 1995).

Research provides support for PBT by documenting the interrelationships among a broad range of risk behaviors (Brener \& Collins, 1998; Khan, Berger, Wells, \& Cleland, 2012; Lindberg, Boggess, \& Williams, 2000; MacArthur et al., 2012; Zweig et al., 2001). Zweig and colleagues (2001), provide an overview of studies that examined patterns of health-risk behaviors. The authors examined the co-occurrence among eight specific behaviors (sexual activity, general alcohol use, binge drinking, cigarette use, marijuana use, other illicit drug use, fighting, and suicide) using data from the National Longitudinal Study of Adolescent to Adult Health (Add Health) collected in 1995. The findings from Zweig and colleagues' study showed that the majority of adolescents in grades 9-12 had low risk profiles. The highest risk profiles indicated links between substance use, sexual activity, violence, and suicide and found a link between sexual activity and substance use supporting previous research. Contrary to previous research indicating that males tend to report higher levels of risk behaviors than females 
(Boggess et al., 2000; Lindberg et al., 2000), Zweig et al found that "only females reported a risk profile that was the highest on every measure of risk, and only females reported a risk profile that had high levels of fighting and suicide with little participation in substance use or sexual activity" (Zweig et al., 2001, p. 724).

Another study using Add Health data examined gender and ethnic differences in the cooccurrence of select adolescent risk behaviors (substance use, early sexual initiation, and truancy) (Weden and Zabin, 2005). This pattern of co-occurrence is consistent with Jessor's 'problem behavior' construct. MacAruthur et al (2012) investigated the patterns of adolescents' risk behaviors by gender and found that while males and females engaged in similar number of behaviors, antisocial and criminal behaviors, marijuana use, motor vehicle related risk behaviors were more prevalent among males and tobacco use, self-harm and sedentary behavior were more common among girls. This study used data from a prospective United Kingdom birth cohort [the Avon Longitudinal Study of Parents and Children (ALSPAC) $]^{5}$.

Although the studies described above contribute to the literature about adolescent risk behaviors and the co-occurrence of risk behaviors among this population, they are limited in two important ways. First, is the changing culture of adolescence in the decade since some of these data were collected (i.e., Add Health data collected in 1995), which may be limiting representativeness of the findings to adolescents today; second, is the limited patterns of risk behaviors studied (e.g., substance use and risky sexual behavior, substance use and violence). This study addresses these two limitations (outdated data and limited number of risk behaviors). Specifically, using recent nationally representative data (2013 YRBSS) a) 40 indicators of health risk behaviors (including depression as a risk factor contirbuting to risky behavior) are measured

\footnotetext{
${ }^{5}$ ALSPAC is a leading birth cohort study - a description of the study can be found at http://www.bristol.ac.uk/alspac/about/
} 
to determine patterns of co-occurrence of these risks in adolescents compared to only eight behaviors studied by Zweig et al (2001), and provide an update on relationships between risks that may have shifted since past studies, b) the relationship between overweight/obesity and risky behaviors in a nationally-representative sample of adolescents are examined. To our knowledge, no studies have examined the association between overweight/obesity and a combination of several risky behaviors among adolescents. Furthermore, the relationship between tobacco use, poor dietary habits and physical inactivity and sedentary behavior are examined.

\subsection{Specific Aims}

The aims of this study were:

Aim 1a: to examine the frequency and co-occurrence of adolescent depression, suicidal thoughts, substance use, behaviors contributing to violence and unintentional injruies, bullying, risky sexual behaviors, unhealthy weight control, poor dietary habits, and physical inactivity and sedentary behaviors. This aim takes a broad view determining the co-occrrence of risky behaviors among adolescents and whether there is a pattern to the co-occurrence of these risk behaviors using a national sample of high school students.

Hypothesis: the risk behaviors and risk factor contributing to risky behavior, (i.e. depression) (listed above) co-occur. Based on the principles of PBT and previous research we hypothesize that substance use, risky sexual behavior, and violent behavior; substance use, depression, suicidal thoughts, and bullying; and tobacco use, unhealthy weight control, poor dietary habits, and physical inactivity are interrelated. PBT proposes that involvement in any one risky behavior increases the likelihood of involvement in other risky behaviors because of their

linkages in the social ecology of youth (socially organized opportunities to learn these behaviors 
and practice them together) and because of what the behavior may mean to adolescents (i.e., expression of independence from parental control) (Jessor \& Jessor, 1977).

Aim 1b: building on the results from 1a, examine whether there are any variations in the pattern of the co-occurrence of adolescent risky behaviors by sex.

Hypothesis: the pattern of risky behaviors and factors contributing to risky behaviors among adolescents vary by sex. This hypothesis was based on the literature demonstrating a higher co-occurrence of, substance use and violent behavior among males; and more prevalance of unhealthy weight control and depression and suicidality among females.

Aim 2: examine the association between overweight/obesity and depression, suicidal thoughts and attempts, substance use (tobacco, alcohol, marijuana, and other illicit drug use), behaviors contributing to violence and unintentional injuries, bullying, risky sexual behaviors, unhealthy weight control, poor dietary habits, and physical inactivity and sedentary behaviors.

Hypothesis: the risk behaviors and risk factor (i.e. depression) listed above are positively associated with overweight and obesity. Based on the principles of PBT and limited previous research on associations of risky behaviors in overweight and obese adolescents, we hypothesize that substance use is positively associated to greater depression and suicidality, bullying, risky sexual behavior, and violent behavior in overweight and obese adolescents and not positively associated among normal weight adolescents

Aim 3: examine the association between tobacco use, poor dietary habits, and physical inactivity and sedentary behaviors among teens, controlling for Body Mass Index (BMI).

Hypothesis: tobacco use is positively associated with poor dietary habits, and physical inactivity in overweight and obese adolescents and not associated in normal weight adolescents. 
To summarize, adolescent health risk behaviors and factors contributing to risk behaviors are a public health concern because they have significant health-related consequences both in the present and in the future. Risky behaviors among adolescents often co-occur. This study will utilize Jessor's (1977) Problem Behavior Theory (PBT) as the theoretical framework to assess the co-occurrence of risky behaviors in a nationally representative sample of adolescents in the United States.

\subsection{Organization of the Study}

The remaining sections of this research report are divided into the following: Chapter 2

contains review of the related literature. Chapters 3-5 present each of the three manuscripts based on this dissertation project, and Chapter 6 presents a summary of important findings, overall discussion, and implications for public health practice and policy. 


\section{Chapter 2}

\section{Literature Review}

This chapter begins with an overview of adolescence and a review of the literature related to adolescent risk behaviors and risk factors contributing to risky behaviors. This is followed by a review of the prominent theories addressing adolescent risk behaviors and a discussion of the theoretical framework used to inform and guide this project (i.e. PBT).

\subsection{Adolescence}

Adolescence is typically characterized by the years between ages 13 and 19 , however the physical and psychological changes that occur during this period can start as early as 10 years old (Sawyer et al., 2012). Adolescence is a life phase during which one experiences not only biological and physical changes but also intellectual, psychological and economic changes (Catalano et al., 2012; Viner et al., 2012; DiClemente et al., 1996). During this phase, individuals reach sexual maturity and make educational and occupational decisions that will guide their careers and futures as adults. It is also a period where future patterns of health behavior are established (Sawyer et al., 2012). Adolescence is often associated with engaging in HRBs such as substance use, violent behavior, suicidality, risky sexual activity, poor dietary habits, and physical inactivity (Catalano et al., 2012; de la Haye, D'Amico, Miles, Ewing, \& Tucker, 2014; Kann et al., 2014; Viner et al., 2012). These lifestyle patterns adopted during adolescence often continue into adulthood, which could potentially influence health outcomes and the development of chronic illnesses (Viner et al., 2012). Therefore, understanding adolescent health-risk behaviors and their co-occurrence is important for policy implementation and for developing effective prevention programs. 


\subsection{Overview of Adolescent Risk Behaviors and Factors}

Research on the health status of United States' youths has shown that the main adolescent health threats are based on their behavioral choices (Resnick et al., 1997; Brener \& Collins, 1998; Kann et al., 2014) that place them at risk for the leading causes of morbidity and mortality (Kann et al., 2014). For example, tobacco, illicit drugs, and alcohol use, violence, risky sexual behaviors, and poor dietary habits could result in poor health and disability, impacting a healthy and productive adult life.

Research has shown that there may be patterns to how adolescent risk behaviors co-occur (Zweig et al., 2001; Zweig et al., 2002). For example, concurrent drug or marijuana use was shown to be associated with smoking onset (Wills, Resko, Ainette, \& Mendoza, 2004) and drug use increased the risk of considering suicide (Park, Schepp, Jang, \& Koo, 2006). In a study by Costello and colleagues, both drug use and delinquent behaviors were related to an increased risk of depressed mood (Costello, Swendsen, Rose, \& Dierker, 2008). Furthermore, research shows that alcohol use is related to early sexual activity, multiple partners, and unprotected sexual intercourse (Fergusson \& Lynskey, 1996; Strachman, Impett, Henson, \& Pentz, 2009). Other unhealthy behaviors that were adopted during adolescence and often continued into adulthood include poor dietary habits, unhealthy weight control, and physical inactivity. These behaviors influence long-term health outcomes and the risk of developing chronic diseases such as diabetes, stroke, coronary heart disease (CHD), and hypertension later in life (Khan et al., 2014; Brener \& Collins, 1998; Resnick et al., 1997).

The Centers for Disease Control and Prevention (CDC) has been monitoring adolescents' risky behaviors over the past two decades through the Youth Risk Behavior Surveillance System (YRBSS). The CDC has identified the following six priority risky behaviors that are the leading 
contributors to morbidity and mortality among youth and young adults in the United States: 1) behaviors that contribute to unintentional injuries and violence; 2) tobacco use; 3) alcohol and other drug use; 4) sexual behaviors that contribute to unintended pregnancy and sexually transmitted infections (STIs), including HIV infection; 5) unhealthy dietary behaviors; and 6) physical inactivity. Research shows that these behaviors often co-occur (Pena et al., 2012; Zweig et al., 2000; Shrier et al., 1997). The co-occurrence of these risky behaviors among adolescents will be discussed throughout the upcoming subsections in this chapter.

\subsection{Adolescent Substance Use}

Substance use constitutes tobacco, alcohol, marijuana, illicit drugs such as cocaine, inhalants (e.g., glue, paints, aerosol spray cans), hallucinogenic drugs (e.g., lysergic acid diethylamide (LSD), phencyclidine (PCP) also known as angel dust, mescaline, or mushrooms), ecstasy, heroin, methamphetamines, and prescription drugs (e.g., steroids, pain killers) (Kann et al., 2014). Much of the information known about adolescent substance use is a result of data collected from national surveys such as the National Health and Nutrition Examination Survey (NHANES); YRBSS, and Monitoring the Future (MTF) study. MTF, a longitudinal study

conducted and monitored by the University of Michigan for the National Institute on Drug Abuse (NIDA), tracks the trend of drug use in adolescents, college students, and adults through age 45 (Johnston, O’Malley, Bachman, \& Schulenberg, 2006).

\section{Tobacco Use}

Tobacco use, especially cigarette smoking, has been identified as a major public health problem (Kann et al., 2014; Centers for Disease Control and Prevention [CDC], 2012). According to the Surgeon General's report smoking impacts nearly every organ in the body and it has been associated with the development of lung cancer, liver and colorectal cancer, as well as many other adverse health outcomes such as cardiovascular and metabolic diseases such as 
diabetes mellitus, pulmonary diseases, impaired immune function, rheumatoid arthritis, and conditions related to pregnancy and birth (Department of Health and Human Services [DHHS], 2014). Smoking tobacco products by children and adolescents hastens the onset of lung function decline during late adolescence and early adulthood. Smoking is also related to impaired lung growth, chronic coughing, and wheezing. In addition, there are adverse impacts such as addiction (i.e., nicotine is highly addictive), lower physical performance (i.e., tobacco narrows blood vessels leading to lack of oxygen and shortness of breath), and negative consequences of secondhand smoke which include cardiovascular disease, bronchitis, and asthma (DHHS, 2014; MacAruthur et al., 2012).

Most adult smokers begin smoking as adolescents (Booker et al., 2004). Data show that nearly nine out of 10 cigarette smokers had tried smoking by age 18 (CDC, 2014). Recent data from the YRBSS show that during the 30 days before the survey, $16 \%$ of high school students had smoked cigarettes and 9\% had used smokeless tobacco. Previous research has shown that tobacco use is related to physical inactivity in youth (Prochaska et al., 2008). There is also some evidence that tobacco use is positively related to unhealthy eating and physical inactivity (Lytle \& Achterberg, 1995; Wayne \& Connolly, 2002), however many of these findings have not been tested among a diverse and representative sample. Studies have shown some evidence that the patterns or risk behaviors differ by gender (MacAruthur et al., 2012), although differences based on other demographics are not known.

Smokeless tobacco (i.e., snuff and chewing tobacco or dip) is also associated with a number of adverse health outcomes, including gingival recession, nicotine addiction, and cancers of the oral cavity, larynx, and pharynx (Wayne \& Connolly, 2002). According to the 2013 YRBSS (Kann et al., 2014), 9\% of U.S. students in grades 9 to 12 reported using smokeless 
tobacco in the 30 days prior to the survey. The use of smokeless tobacco is highest among white male students than it is among black and Hispanic male students $(21 \%, 4 \%$, and $8 \%$, respectively). More recently, tobacco manufacturers have introduced a proliferation of colorful brands featuring candy-like flavors (e.g., Dark Mint, Mocha Taboo, Midnight Berry) as well as products that are smokeless and spitless (i.e., Marlboro Snus comes in a teabag-like pouch that a user puts between the cheek and gum and then discards after about 30 minutes) in order to appeal to youth. A study by Carpenter and colleagues (2005) aimed to assess the role of flavored cigarettes in the targeting of adolescents. The study found that there is a link between these youth targeted products and smoking behavior (Carpenter, Wayne, Pauly, Koh, \& Connolly, 2005). For example, these exotic flavored cigarettes can promote youth tobacco use initiation and may push social smokers to become daily smokers by downplaying or masking the taste of tobacco smoke (Wayne \& Connolly, 2002). The potential added health risks associated with these new more exotic brands, often portrayed as a "healthier" alternative, have not been thoroughly studied and thus are cause for concern.

\section{Alcohol, Marijuana and Other Illicit Drug Use}

The use of alcohol, marijuana, and other illicit drugs among adolescents are associated with morbidity and mortality. These substances are not only associated with negative health outcomes but also adverse social and economic impacts including family dysfunction, delinquency, school dropout and unemployment (de la Haye et al., 2014).

\section{Alcohol Use}

Alcohol use/abuse has been linked to a range of negative health behaviors and outcomes, including death. For example, alcohol abuse has been associated with unintentional injuries, risky sexual behavior, aggressive behavior, poor performance in school or at work, use of other illicit drugs, depression, and suicidal ideation and attempts (CDC, 2012; Hallfors et al., 2005). 
Data show that nationwide, $66 \%$ of students had at least one drink of alcohol on at least one day during their life. The prevalence of having ever had a drink was higher among females than it was males (68\% and 64\%, respectively). Data also show racial disparity in the prevalence of alcohol with Hispanic students having the highest prevalence of ever having drunk alcohol than white and black students $(72 \%, 66 \%$, and 63\%, respectively) (Kann et al., 2014). According to the $\mathrm{CDC}$, the prevalence of current alcohol use (i.e., at least one drink of alcohol on at least 1 day during the 30 days before the survey) among U.S high school students was 35\%, with white and Hispanic students having the highest prevalence than black students $(36 \%, 37 \%$, and $30 \%$, respectively) (Kann et al., 2014). Research has shown an association between alcohol use and other HRBs among adolescents such as risky sexual behavior (Brener \& Collins, 1998;

Fergusson \& Lynskey, 1996; Khan, Berger, Wells, \& Cleland, 2012; Strachman et al., 2009; Zweig et al., 2001; Zweig et al., 2002), mental health problems including depression and suicidality (Brener \& Collins, 1998; de la Haye et al., 2014), and behaviors resulting in injury including drunk driving (Basen-Engquist, Edmundson, \& Parcel, 1996; Dukarm, Byrd, Auinger, \& Weitzman, 1996; Jessor \& Jessor, 1977) and violence such as weapon carrying and physical fights (Dukarm et al., 1996). However, interestingly, limited research has been done on the cooccurrence of alcohol abuse and poor dietary behavior, sedentary behavior, and overweight/obesity. In Jessor's research these behaviors were considered health compromising behavior, however poor dietary behavior, sedentary behavior and obesity are far more prevalent now than in the 1970 s and 1980 s with significant contribution to morbidity among this population. Hence, the inclusion of these behaviors as risky behaviors in our study.

\section{Marijuana Use}

According to the CDC, $41 \%$ of high school students reported having ever used marijuana (i.e., one or more time during their life). This prevalence was higher among male than female 
students (42\% and 40\%, respectively). Racial/ethnic differences exist in the use of marijuana among high school students with black and Hispanic students having a higher prevalence than do white students (47\%, 49\%, and 37\%, respectively) (Kann et al., 2014). Marijuana use has been associated with depression (Dukarm et al., 1996; Jessor, Chase, \& Donovan, 1980; Jessor \& Jessor, 1977; Jessor, Van Den Bos, Vanderryn, Costa, \& Turbin, 1995), delinquency (Dukarm et al., 1996; Farrell, Danish, \& Howard, 1992), and violent behavior (Dukarm et al., 1996; Farrell et al., 1992; Jessor et al., 1980; Jessor \& Jessor, 1977).

\section{Illicit Drug Use}

Nationwide, the prevalence of other illicit drug use among adolescents are as follows; $5 \%$ of adolescents report having ever used any form of cocaine (i.e., powder, crack), $7 \%$ have used hallucinogenic drugs (e.g., LSD, PCP, acid), 9\% have used inhalants (e.g., sniffed glue, breathed the contents of aerosol spray cans), $7 \%$ have used ecstasy (also called MDMA), $2 \%$ have used heroin, 3\% have used methamphetamines (also called speed, crank, ice), and $18 \%$ have taken prescription drugs (e.g., Oxycontin, Percocet, Vicodin, Adderall, and Xanax) without a doctor's prescription one or more times during their life.

A study by Dukarm and colleagues showed that a significant increase in the number of male and female adolescents carrying weapons and physically fighting was associated with all forms of substance use including alcohol consumption ( $34 \%$ vs $17 \%, \mathrm{P}<.001)$, marijuana use (48\% vs $22 \%, \mathrm{P}<.001)$, cocaine (71\% vs $25 \%, \mathrm{P}<.001)$, and anabolic steroids $(62 \%$ vs $25 \%$, $\mathrm{P}<.001)$ (Dukarm et al., 1996).

\subsection{Behaviors that Contribute to Unintentional Injuries}

Unintentional injuries are a major source of premature morbidity and mortality for children and adolescents in the United States. Unintentional injuries are the leading cause of death in adolescents (Sleet et al., 2010). Although there are different causes of unintentional 
injuries, motor vehicle crashes (MVCs), in particular, represent the biggest burden of injury and death to adolescents and young adults (Jelalian, Alday, Spirito, Rasile, \& Nobile, 2000). Other major causes of unintentional injuries include falls, poisoning, drowning, fire and burns, and recreational and sports accidents (CDC, 2014). Recent data from CDC indicate that MVCs account for more than half (65\%) of the causes of deaths from unintentional injuries for adolescents ages 15-19 years (CDC, 2014).

Adolescent risky behaviors may underlie many MVCs. Research shows that measures of risk-taking and risky driving practices are highly correlated. Adolescents who have ridden with a drunk driver are less likely to use a seat belt, more likely to smoke, more likely to drink alcohol, more likely to drink alcohol and drive, and more likely to engage in risky sexual activity (Petridou et al., 1997). Recent national data from YRBSS indicate that " $41.4 \%$ of high school students nationwide among the $64.7 \%$ who drove a car or other vehicle during the 30 days before the survey had texted or e-mailed while driving, $34.9 \%$ had drunk alcohol, and $23.4 \%$ had used marijuana." (Kann et al., 2014, p. 1). Nationally, 7.6\% of adolescents never or rarely wore a seat belt when riding with someone else, this may be a huge contributor to the rate of mortality and morbidity from MVCs in this population.

\subsection{Behaviors that Contribute to Violence}

Violence has become one of the leading causes of death in adolescents. According to the CDC, an estimated 55,000 people die annually in the U.S. as a result of violence-related injuries (CDC, 2014). Homicide is the second leading cause of death for youth and young adults aged 1524 years and the fourth leading cause of death for those aged 10-14 years (Parks, Johnson, McDaniel, \& Gladden, 2014). Homicide is defined as a death that results from the use of 
physical force, threatened or actual, against another person, group, or community when evidence indicates that the use of force was intentional (Parks et al., 2014).

Research has identified the increase in poverty, family violence, substance use, and easy access to firearms as the risk factors for the increase in violence in this population (Dukarm et al., 1996; Duke \& Borowsky, 2015; Parks et al., 2014). Physical fights are often a precursor to other types of violent encounters. The most recent data indicate that 1 in 4 high school students reported being in at least one physical fight, and 1 in 12 high school students reported fighting at least once on school property in the past year (Kann et al., 2014). Research has shown an association between substance use and aggression and behaviors that could result in injury (intentional and/or unintentional) (Connell, Gilreath, \& Hansen, 2009; Jelalian et al., 2000; Petridou et al., 1997).

The risk of some of the forms of violence discussed above varies significantly by racial and ethnic groups. For example, the homicide rate for African American youth was almost 14 times higher than the rate for white youth and 14 times higher than the rate for Asian/Pacific Islander youth. The homicide rate for non-Hispanic African American youth was also 4.1 times higher than the rate for Hispanic youth and 3.8 times higher than the rate for non-Hispanic American Indian/Alaska Native youth. Homicide is the leading cause of death for non-Hispanic African-American youth, the second leading cause of death for Hispanic youth, the third leading cause of death for non-Hispanic American Indian/Alaska Native (CDC, 2014).

The prevalence of some types of violence among youth also varies by sex. For instance, the youth homicide rate in 2011 was 6 times higher among males $(12.3$ per 100,000) than among females (2.1 per 100,000). The rate of involvement in physical fights among high school students also was approximately 1.6 times higher for male (30\%) compared to female students (19\%). 
Data show violence is also a critical public health issue for young females. Among females aged 10-24 years, homicide is the fourth leading cause of death (CDC, 2014).

Youth violence also occurs in schools and it can adversely affect students' ability to attend and fully participate in school activities. In 2013, 20\% of high school students reported being bullied at school, and 7\% reported being threatened or injured with a weapon on school property in the past 12 months (Kann et al., 2014).

\section{Bullying}

Bullying is defined as unwanted, aggressive behavior among school aged children that involves a real or perceived power imbalance between the victim and the perpetrators (J. Wang, Nansel, \& Iannotti, 2011). Bullying includes actions such as making threats, spreading rumors, attacking someone physically or verbally, and excluding someone from a group on purpose (Spriggs, Iannotti, Nansel, \& Haynie, 2007). Cyberbullying is a type of bullying that takes place using electronic technology. Examples include, mean text messages or emails, rumors sent by email or posted on social networking sites, and embarrassing pictures, videos, websites, or fake profiles (Spriggs et al., 2007).

In recent years, bullying has been identified as a public health issue in the United States because of its high prevalence and severe consequences. Bullying can have an effect not only on those who are bullied but also those who bully, and those who witness bullying. Bullying is linked to many negative outcomes including impacts on mental health, substance use, and suicide (Spriggs et al., 2007). Recent data show that nationwide 20\% of adolescents reported having been bullied on school property (Kann et al., 2014). In addition to associations with other forms of violent behaviors (e.g., weapon carrying, suicidality), bullying is associated with substance use, and mental and physical health symptoms (Spriggs et al., 2007; Nansel et al., 2001; Kaltiala-Heino et al., 2000). 
Research found that boys have a higher prevalence of being perpetrators of bullying than do girls (Wang, Iannotti, \& Nansel, 2009). In a nationally representative study of U.S. adolescents, Nansel and colleagues (2001) reported that the prevalence of involvement in school bullying in the past two months was $30 \%$, with $13 \%$ being bullies, $11 \%$ being victims, and $6 \%$ being both (Nansel et al., 2001). Studies have shown that boys are more likely to be involved in direct form of bullying which constitutes hitting, pushing, and kicking, and verbal bullying, whereas girls are more involved in indirect form of bullying which includes social exclusions and spreading rumors (Spriggs et al., 2007; Nansel et al., 2001; Kaltiala-Heino et al., 2000, Wang et al., 2009).

Data from the 2013 national YRBSS indicate that 15\% of adolescents had been bullied electronically (Kann et al., 2014). Although the cyber world provides many benefits such as social support, opportunities for cross-cultural interactions, and identity exploration for adolescents, it also puts them at risk for cyber bullying (Mishna et al., 2012). Mishna and colleagues (2012) found that students who have been cyber bullied have reported feeling sad, anxious, unable to concentrate on school work and exhibit social difficulties, substance use and eating disorders. No gender differences were found among students who bullied others or who were victimized online. Interestingly, the only difference found between males and females in Mishna and colleagues' study was whether the teens identified themselves as both bully and victim. Females were more likely than males to be bully-victims. Mishna et al (2012) explain that "since females tend to use indirect bullying more frequently than males, cyber, which in many ways is considered indirect, may provide an opportunity for females to engage in more aggressive behaviors" (p. 67). 


\subsection{Adolescent Risky Sexual Behavior}

Risky sexual behaviors are those which increase the risk of contracting or transmitting disease, or increase the occurrence of unwanted pregnancy. Risky sexual behaviors include having more than one sexual partner, promiscuity, having oral, vaginal or anal sexual contact without a condom, using unreliable methods of birth control, or using birth control inconsistently (Kann et al., 2014).

According to the CDC, recent data indicate that nationally, $46.8 \%$ of high school students had ever had sexual intercourse. Overall this percentage was higher among black male (68.4\%) and Hispanic male (51.7\%) than black female (53.4\%) and Hispanic female (46.9\%) students. Not surprisingly, 11 and 12 graders were significantly more likely than students in grades $9^{\text {th }}$ and $10^{\text {th }}$ to report having had sexual intercourse (CDC, 2014).

Adolescents who engage in unprotected sexual activity are at risk for sexuallytransmitted infections (STIs) including HIV, and unwanted pregnancy, which amplify some of the concerns related to sexual risk behaviors. Contracting STIs during adolescence can have significant negative consequences for reproductive health. STIs can cause pelvic inflammatory disease, infertility, ectopic pregnancy, preterm births, and fetal abnormalities (Hamilton, Martin, \& Ventura, 2010). African American and Hispanic youth and young adults between the ages of 14 to 25 have higher incidences of STIs such as Chlamydia and increased number of new cases of HIV/AIDs, and higher rates of pregnancy compared to non-Hispanic white youth (Yan, Chiu, Stoesen, \& Wang, 2007). Recent data indicate an estimated 329,772 births; 548,032 cases of chlamydia, gonorrhea, and syphilis; and 2,240 cases of HIV annually among youth aged 15-19 years (Kann et al., 2014). 


\section{Teen Pregnancy}

Adolescents' engagement in risky sexual behaviors contribute to unwanted pregnancy in addition to high risks for sexually transmitted diseases. The United States has the highest adolescent pregnancy rate of all industrialized nations, 34.2 births per 1,000 women ages 15-19 compared to the Netherlands with only 5.3 births per 1,000 women ages 15-19 (The World Bank, 2015). There are regional differences within the country such that adolescent birth rates are lowest in the Northeast and upper Midwest and highest across the South and Southwest U.S. (Hamilton, Martin, \& Ventura, 2010). There can be numerous reasons for these disparities, including the proportion of adolescents who are sexually active and ultimately at risk for STI or unwanted pregnancy, variation among sexually active adolescents in taking steps to prevent STI infections and unwanted pregnancy, including method of contraceptive and its effective use, effective sexual health education, and access to contraceptives.

Long-term consequences of adolescent pregnancy include lower educational attainment, lower socioeconomic status, psychological difficulties, and higher rates of single parenthood (McCave $\&$ Shiflet, 2010; Mahfouz et al., 1995). Moreover, adolescent motherhood may compound these socioeconomic disadvantages by increasing the risks of additional adverse socioeconomic problems as well as poor health outcomes for the mother and the infant (McCave \& Shiflet, 2010). Infants born to adolescent mothers are at risk for prematurity, low birth weight, small for gestational age (SGA), and neonatal mortality (Black, Fleming, \& Rome, 2012; Fleming, Tu, \& Black, 2012). These poor health outcomes may be explained by possible biological factors and psychological immaturity for reproduction (Black et al., 2012) in addition to the socioeconomic factors (Black et al., 2012; Fleming et al., 2012). Some researchers argue that biological factors such as age or maternal growth are not a risk factor, and that unfavorable outcomes are more likely associated with socioeconomic and lifestyle factors (Mahfouz, El-Said, Al-Erian, \& 
Hamid, 1995; Rogers, Peoples-Sheps, \& Suchindran, 1996). However, other researchers have found that adolescents have higher rates of adverse pregnancy outcomes compared to adult women even after controlling for socioeconomic factors (Alves, Cisneiros, Dutra, \& Pinto, 2012; Black et al., 2012; Chen et al., 2007; Fleming et al., 2012).

As discussed above adolescence is also a period of vulnerability for substance use (alcohol, tobacco, and other drug use) (CDC, 2012; Khan et al., 2012; Horn et al., 2005). Substance use increases youths' risk of engaging in risky sexual behaviors (multiple partners, unprotected sexual intercourse) and becoming pregnant (Khan et al., 2012; Strachman et al., 2009). Research illustrate that drug and alcohol use diminish both inhibitions and rational decision making leading to involvement in risky behaviors including risky sexual behaviors (Fergusson \& Lynskey, 1996; Strachman et al., 2009). A study by Lammers and colleagues (2000) found that among 26,023 adolescents aged 13-18 years old, substance use was significantly associated with the initiation of sexual intercourse (Lammers, Ireland, Resnick, \& Blum, 2000). A longitudinal study, examining the relationship between adolescent substance use and risky sexual behavior in late adolescence and young adulthood, found that earlier age of onset of sexual activity, promiscuity, and inconsistent condom use were reported by those adolescents that also reported substance use (Tapert, Aarons, Sedlar, \& Brown, 2001). Understanding the co-occurrence of these risk behaviors among adolescents will help direct efforts to addressing adolescents' willingness and participation in risk behaviors in order to help educate them in choosing healthier paths that will help them and the society long term.

\subsection{Adolescent Mental Health}

\section{Depression and Suicidality}

Depression inflicts significant negative outcomes both medically and socially, ranking fourth in terms of its contribution to the global burden of disease (World Health Organization, 
n.d.). Research shows that depression has become more common in adolescence over time (Park et al., 2006; Jiang, Perry, \& Hesser, 2010).

Depression experienced during adolescence may adversely impact growth and development, family and peer relationships, and academic performance as well as health outcomes in late adolescence and adulthood (Bhatia \& Bhatia, 2007). According to research by Costello et al (2002), the median prevalence of depression is below $2 \%$ in childhood. However, during adolescence and young adulthood, this prevalence increases to $12 \%$ for major depression and $7 \%$ for minor depression. The Diagnostic and Statistical Manual of Mental Disorders (DSM-IV) defines major depressive disorder as experiencing either depressed mood or loss of interest/pleasure and at least four other symptoms that reflect a change in functioning, such as problems with sleeping, eating, energy, concentration, and/or self-image, for a period of two weeks or longer; and minor depressive disorder as having at least two depressive symptoms present for two weeks. Minor depressive disorder or minor depression is a mood disorder that does not meet full criteria for major depressive disorder but in which at least two of the above depressive symptoms are present for two weeks.

Data from the 2013 YRBSS show that 29\% of U.S. high school students reported severe depressive feelings, $17 \%$ had seriously considered attempting suicide, and $8 \%$ had attempted suicide at least once in the preceding year. Female students had a higher prevalence of having seriously considered attempting suicide $(22 \%)$ than did male students $(12 \%)$. Female students in grades ninth, tenth, and eleventh $(25 \%, 23 \%$, and $22 \%$, respectively) were significantly more likely than were twelfth graders $(18 \%)$ to have attempted suicide in the past year. Hispanic females were significantly more likely to attempt suicide (26\%) than African Americans (18\%) and Whites females (21\%) (Kann et al., 2014). 
Research has linked early initiation of tobacco, alcohol and other illicit drug use with depression, addiction, and other psychiatric disorders in adulthood regardless of sociodemographic factors such as age, gender, family income, and parental education (Brook et al., 2002; Aseltine et al., 2009; Joe et al., 2007). The relationship between cigarette smoking and depression has been explained in several ways. Munafo and colleagues (2008), using nationally representative data from the Longitudinal Study of Adolescent to Adult Health, showed that among individuals who had not smoked cigarettes at baseline, increases in depressive symptoms lead to smoking initiation. The idea of self-medication is another hypothesis which purports that individuals use certain substances as a method to alleviate their anxiety and distress. Minnix and colleagues (2011) note that individuals who have low self-efficacy with respect to controlling their emotional states may be more vulnerable to depressive symptoms which may in turn lead to use of tobacco products to control depressive symptoms.

Others suggest that depression increases adolescents' likelihood of becoming smokers by increasing susceptibility to peer pressure from those who are already smokers (Patton et al., 1998; Morrell, Cohen, \& McChargue, 2010). Moreover, research shows higher levels of depression among adolescents who are current smokers compared to those who are nonsmokers and former smokers (Martini, Wagner, \& Anthony, 2002), leading some to hypothesize that cigarette smoking may lead to depression. Others suggest that depression increases adolescents' likelihood of becoming smokers by increasing susceptibility to peer pressure from those who are already smokers (Patton et al., 1998; Morrell, Cohen, \& McChargue, 2010). A study using longitudinal data found that even after controlling for prior level of depression, adolescents who were smokers were more likely to experience depressive symptoms (Steuber \& Danner, 2006). More research is needed to explain the mechanisms responsible for the relationship between 
cigarette smoking and depressive symptoms, however, we know through extensive research that a relationship exists between smoking and depression and our study shows these behaviors tend to co-occur in this population. The association between alcohol use and depression is also well documented (Aseltine et al., 2009; Botticello, 2006; Kessler et al., 1996; Regier et al., 1990; Helzer \& Pryzbeck, 1988).

\subsection{Adolescent Obesity, Poor Dietary Behaviors, and Sedentary Behaviors}

Obesity has become increasingly prevalent in the past few decades, affecting individuals in all age and race/ethnic groups in the United States. Substantial increases in the rate of overweight obesity in childhood and adolescence have been documented. Nationally, 32\% of 219 year old children are overweight or obese (Ogden et al., 2014).

Childhood obesity is a significant health risk with detrimental long term physical, psychological, and economic consequences (Anuradha et el., 2015). The immediate health effects of obesity in youth include risk factors for cardiovascular disease, such as high cholesterol or high blood pressure. In addition, obese adolescents are more likely to have prediabetes, a condition in which blood glucose levels indicate a high risk for development of diabetes (Ogden et al., 2014). Also, children and adolescents who are obese are at much greater risk for bone and joint problems, sleep apnea, and social and psychological problems such as poor self-esteem and stigmatization (Ogden et al., 2014).

Obesity in childhood and adolescence is likely to track into adulthood (Reilly et al., 2003; Singh, Mulder, Twisk, Van Mechelen, \& Chinapaw, 2008) and has multiple negative long-term outcomes (Anuradha et al., 2015; Reilly et al., 2003; Singh et al., 2008). Early literature reviews (Must et al., 1999) and more recent systematic reviews of the literature (Park, Falconer, Viner, \& Kinra, 2012; Reilly \& Kelly, 2011) suggest increased risk of cardio-metabolic morbidity (e.g., 
diabetes, stroke, coronary heart disease, hypertension) and all-cause mortality in adulthood among those who were overweight or obese during childhood (Baker, Olsen, \& Sørensen, 2007).

Although complications and adverse health effects of childhood overweight and obesity are well documented and include metabolic health risk, chronic diseases, psychosocial issues (Ebbeling, Pawlak, \& Ludwig, 2002; Ludwig, 2007), much less is known about the association of overweight/obesity with other health-risk behaviors, which are also considered primary threats to adolescent health and wellbeing. Overweight/obese adolescents are particularly vulnerable to risky behavior compared to their normal weight peers, because overweight/obese youth are more likely to experience impaired peer relationships, stigmatization, and weight bias (Farhat, Iannotti, \& Simons-Morton, 2010). The combination of these stressful events, the normative challenges of adolescence, along with the stress of managing an unhealthy weight, may contribute to the susceptibility of these adolescents in engaging in risky behaviors (Booker, Gallaher, Unger, RittOlson, \& Johnson, 2004).

Research shows considerable gender and racial/ethnic disparities in obesity among children and adolescents (Farhat et al., 2010; Kann et al., 2014). Non-Hispanic white children and adolescents have the lowest prevalence compared with their non-Hispanic black and Mexican-American counterparts (Wang \& Zhang, 2006). This study is guided by PBT, in which individual, biological, behavioral, and personality factors interact with perceived and actual social environments, predisposing adolescents to and protecting them from health risk behaviors depending on the factors present.

\subsection{Theories of Adolescent Risk Behaviors}

The literature review of adolescent risky behaviors in the previous section provided information about the important behavioral factors contributing to morbidity and mortality of 
adolescents in the United States. In order to have an understanding of health behaviors and the situations in which they occur or co-occur, it is important to consider the use of health behavior theories, which focus on different determinants of behavior at individual, interpersonal, and community levels (Glanz, Rimer, \& Viswanath, 2008). A growing body of evidence suggests that research, health promotion and education initiatives, and interventions designed or developed with a theoretical base or foundation are more effective than are those lacking a theoretical base (Glanz \& Bishop, 2010; Glanz et al., 2008; Painter, Borba, Hynes, Mays, \& Glanz, 2008). Painter and colleagues (2008) found that in their sample of 193 health behavior articles 35\% used a theoretical framework and among articles that used at least one theory 59\% of the studies were informed by theory. For example, a meta-analysis of almost 200 intervention studies to increase condom use found that many constructs in commonly used health behavior theories were successfully changed by interventions, and some of these constructs even mediated changes in condom use (Albarracín et al., 2005).

Given the aims of this study and the nature of the dataset (i.e., YRBSS - secondary individual level behavioral data), utilization of a theoretical framework is useful in two ways. First, a theoretical framework provides comprehensive conceptual understanding of factors associated with adolescent risk behaviors. Second, the use of theory provides a different "lens" through which to look at a complicated problem or set of problems (i.e. adolescent risky behaviors), focuses on different aspects of the data, (i.e., variable selection), and provides a framework to conduct appropriate analyses.

Glanz and Bishop define theory as "a systematic way of understanding events, behaviors and/or situations. A theory is a set of interrelated concepts, definitions, and propositions that explains or predicts events or situations by specifying relations among variables." (Glanz \& 
Bishop, 2010, p. 401). Glanz and Bishop further explain that theories are abstract in order to be applicable to a broad range of topics and contents. For example, theories can guide research to better understand why adolescents do or do not engage in risky behaviors. Theories can also be used to suggest how to develop more effective ways of promoting and affecting behavior change. For example, understanding why adolescents engage in risky sexual behavior is one step closer to developing prevention efforts.

In order to provide context for the selection of the theoretical framework most appropriate for this study, we describe the three most widely used theories in adolescent risk/problem behaviors and discuss the shared elements of these theories.

\section{Social Learning/Social Cognitive Theory}

Social learning theory posits that "learning_is a cognitive process that takes place in a social context and can occur purely through observation or direct instruction, even in the absence of motor reproduction or direct reinforcement." (Bandura \& McClelland, 1977, p. 25). Almost a decade later, Bandura expanded and renamed his theory as social cognitive theory (SCT), emphasizing the major role cognition plays in behavior (Bandura, 1986). SCT stresses that self-efficacy mediates the effects of other variables as well. Self-efficacy refers to an individual's belief in his or her capacity to execute behaviors necessary to produce specific performance attainments (Bandura, 1986). SCT has evolved from social learning theory to address both the underlying determinants of health behavior as well as methods of promoting change (Bandura, 1986). This theory stresses the complexity of the relationship between individuals and their environment. Bandura explains that the interaction between personal characteristics, behavior, and environment are "reciprocal and dynamic." This interaction also reflects confidence in one's ability to exert control over one's own motivation, behavior, and social environment. SCT is an important theory in addressing adolescent behavior because its 
key constructs include observational learning, reinforcement, self-control, and self-efficacy. For example, evidence suggests that adolescents engage in unhealthy weight control activities such as the use of laxatives and/or vomiting, diet pills, or fasting as a means to lose weight (Kilpatrick, Ohannessian, \& Bartholomew, 1999), research also indicates that health education in the classroom provides opportunities to adolescents to modify and improve health behavior (Hallfors et al., 2004; Marshall, Biddle, Gorely, Cameron, \& Murdey, 2004). Elements from the SCT could be used to explain classroom education positively impacts health behavior in adolescents by accounting for the interaction between behavior, personal factors, and environmental influences (i.e., knowledge and skills to perform a given behavior, anticipated outcomes, values placed on a given outcome).

\section{Theory of Planned Behavior}

Similar to SCT, the theory of planned behavior (TPB) is one of the theories that emphasizes the role of cognition in behavior. This theory extends the Theory of Reasoned Action (TRA) by adding perceived behavioral control (PBC) as a determinant of behavior and behavioral intentions (Ajzen, 1991). TRA is a theoretical model for the prediction of behavioral intention, spanning predictions of attitude and behavior. The subsequent separation of behavioral intention from behavior allows for explanation of limiting factors on attitudinal influence (Ajzen, 1980). TPB is an integration of rational decision-making theories (i.e. a multi-step process for making choices between alternatives), such as the TRA theory of reasoned action, and those that emphasize perceived behavioral control (i.e. adolescent's assessment of his/her ability to act on intentions given personal resources and situational constraints), such as in SCT (Ajzen, 1991, 2011; Pickett et al., 2012).

The central hypothesis of TPB is that behaviors are determined by "behavioral intentions." In essence, this theory incorporates three main constructs that contribute to 
behavioral intentions (Ajzen, 1991). Intentions are assumed to reflect willingness and motivation to participate in certain behaviors. This theory could be useful in identifying appropriate information to collect from a target population before developing awareness campaigns, interventions, or prevention programs. The theory highlights the need to understand the beliefs of the target population about the issue, who they see as affecting these beliefs and behaviors, and perceived barriers to taking actions that might promote healthy behavior. This theory has widely been used in the development of programs to reduce uptake of smoking among youth (Ajzen, 1991) and obesity prevention and dietary control interventions (Hackman \& Knowlden, 2014). The TPB constructs have been used to inform risky behaviors such as risky sexual behaviors (i.e., unprotected sex), smoking, and alcohol use. For example, Jemmott and colleagues (2007) conducted a study where they drew upon TPB to identify the modifiable determinants of intention to use condoms among a sample of South African adolescents. The participants included $3906^{\text {th }}$ grade students in public schools in Mdantsane, South Africa who completed anonymous questionnaires. The analyses revealed that attitudes and perceived behavioral control were significantly related to the intention to use condoms. Jemmott et al (2007), concluded that TPB can help explain the social psychological dynamics of an important sexual risk behavior among the Xhosa-speaking adolescents there were the participants of their study. Another study by Kuther and Higgins (2003) demonstrated that among high school students, college freshmen, and junior students subjective norms especially peer norms were a predictor of alcohol use whereas, parental norms were not a predictor of alcohol use among these three groups of students (Kuther \& Higgins-D'Alessandro, 2003). Their findings were consistent with other studies, suggesting that peers may influence alcohol consumption through modeling drinking behaviors 
(Barnes, Farrell, \& Banerjee, 1994; Hawkins, Catalano, \& Miller, 1992; Kandel, 1985), and attitudes toward alcohol use (Ary, Tildesley, Hops, \& Andrews, 1993; Barnes et al., 1994).

Because the TPB is a theory of behavior that results from rational planning, not all health behaviors may be explained by its principles and more experimental work remains to be done on the mediating relationships that the model proposes.

\section{Problem Behavior Theory}

Similar to SCT and TPB, Jessor's problem behavior theory (PBT) postulates that all behavior is the result of person-environment interaction (Basen-Engquist et al., 1996; Jessor, 1991, 1992; Jessor \& Jessor, 1977). In the early development of the PBT, a combination of six behaviors represented actions which reflected a syndrome - a clustering of co-occurring signs or symptoms (Allen, Leadbeater, \& Aber, 1994), rather than individual behaviors occurring at random in the same individual. Those behaviors included activism/social protest, drug use, sexual behaviors, alcohol use, problem drinking, and general deviant behaviors. PBT was expanded further to encompass prosocial behavior, and health compromising and healthenhancing behaviors (Jessor et al., 1995).

PBT conceptualizes that behavior emerges out of the structure and interaction of three systems (Jessor, 1991; Jessor et al., 1995), and each system is comprised of interrelated variables which provide an explanation for a greater or lesser likelihood of problem behavior. The three systems are explained by Jessor and colleagues as the behavior system, personality system, and perceived environment system. The behavior system includes both problem and conventional behavior structures. Risky behaviors such as alcohol use, binge drinking, cigarette use, marijuana and other illicit drug use, risky driving, unhealthy weight control, risky sexual behaviors, and other deviant/delinquent behaviors fall under this system. Given that our focus is on the cooccurrence of health risk behaviors among adolescents, the behavior system of PBT is the most 
relevant system to this dissertation project. Jessor proposes that involvement in any one problem behavior increases the likelihood of involvement in other problem behaviors mainly because of their linkages in the social ecology of youth—socially organized opportunities to learn and to practice them together-and to the similar psychological meanings and functions the behavior may have (for example expression of independence from parental control).

The personality system involves a combination of the motivational-instigation structure, which provides motivational sources or pressures to goal attainment, the personal belief structure, which provides restraint on engaging in nonconformity that originate in beliefs about self and society, and the person control structure, which is concerned with control against nonnormative behavior. The personal belief structure includes social criticism, alienation, selfesteem, and internal-external locus of control (Jessor, 1992). Jessor further explains that problem behavior often results from personality patterns related to low achievement, focus on independence, favorable attitudes towards deviancy, adoption of values that are counter to social expectations and lower self-esteem (Jessor, 1991, 1992).

The perceived environment system includes two structures: distal, inclusive of a person's relationship to their support network (for example, parental support); and proximal, which deals with a person's environment in relation to available models of behavior (for example, peer models for alcohol use). Proximal variables directly implicate a particular behavior whereas distal variables are more distant and require theoretical linkage to behavior (Costa, 2005). Problem behavior in the environment often elicits high peer approval, peer models, low parental control, support and influence, and incompatibility between parent and peer expectations. PBT holds that when the personality system and perceived environment system clash, behavioral problems become apparent (Jessor, 1991, 1992). To further illustrate, prominent features of 
adolescence such as "impulsivity, risk-taking, perceived invulnerability, struggling to find personal identity, errors in thinking due to being locked into normative peer culture, and rebellion towards authority" (Jessor 1991, p. 599), along with the disturbances in psychosocial adjustment, clash with the norms and expectations of the society and as a consequence could result in problem behavior. As is evident from the discussion above the personality and perceived environment systems are beyond the scope of this project. This research study will focus on the co-occurrence of health risk behaviors among adolescents. Therefore, we will use constructs from the behavior system of PBT as a framework in selecting our variables of interest and formulating hypotheses to our research questions. Future research, investigating the psychosocial and environmental contexts to adolescent engagement in risky behavior may use variables representing all three systems.

\section{Empirical Support for PBT}

PBT has been employed in both cross-sectional (Donovan, 1996; Jessor et al., 1980) and longitudinal (Donovan, Jessor, \& Costa, 1999) studies. Jessor and Jessor (1977) conducted longitudinal studies of over 600 youth ages 12 to 22 years old in order to provide empirical support for utilization of PBT to explain adolescent problem behavior.

In a cross-sectional study, Jessor and colleagues assessed personality, environment, and behavioral variables representing psychosocial risk factors for adolescent problem behaviors in 10,000 junior and senior high school students (Jessor et al., 1980). This study found significant correlations with marijuana use and the relationships held across differences in age, sex, and race/ethnic membership. Multiple regression analyses performed in the study showed that this pattern of psychosocial correlates accounted for over 50 percent of the variation in marijuana use. Other researchers used psychosocial concepts and measures derived from PBT to investigate both the occurrence and co-occurrence of a variety of behaviors in childhood, adolescence, and 
young adulthood, including alcohol use, tobacco use, early sexual intercourse, risky driving behaviors and illicit drug use (Donovan, 1996; Donovan et al., 1999; Jessor et al., 1980; Zweig et al., 2001; Zweig et al., 2002). For example, Jessor and colleagues (1980) used PBT as a framework, and found that "greater involvement in marijuana use was associated with greater value on independence than on academic achievement, greater tolerance of deviance, less compatibility with parents, less involvement in conventional behavior such as attending church, and greater actual involvement in other problem behaviors such as drunkenness." (Jessor et al., 1980, p. 604). A relatively recent study used cluster analysis to determine the interrelationship between eight health risk behaviors (sexual activity, general alcohol use, binge drinking, cigarette use, marijuana use, other illicit drug use, fighting, and suicide) and found distinct clusters by gender based on their profiles of risk-taking behavior (Zweig et al., 2001). Zweig et al (2001) found high risk profiles of alcohol use and sexuality in females, and high risk profiles of marijuana use and suicidal behaviors.

One of the limitations of the earlier model of PBT was that it did not explicitly outline the implications of subpopulation differences (i.e., gender, race/ethnicity). However in the later model, Jessor clarifies and includes constructs both related to health (e.g., chronic disease, mental health stressors) and those related to socioeconomic well-being (e.g., school failure, unplanned pregnancy) (Donovan, 1995; Jessor et al., 1995). The later model of PBT incorporates elements of the macro context discussed in social control theory (Umberson, 1992), and interpersonal interactions involved in social learning theory (Bandura \& McClelland, 1977), and the personality and biological components of sensation-seeking models (Zuckerman, 1984) in order to allow for an understanding of the behavioral differences by gender and ethnicity (Weden \& Zabin, 2005). For example, Weden and Zabin (2005) use constructs of PBT to examine gender 
and ethnic differences in the co-occurrence of adolescent health-related behaviors. These researchers found that for all ethnic groups, there was a subpopulation with problem behavior characteristics such as early sexual initiation, alcohol use, smoking, marijuana use, and truancy. Further, this study found that truancy, early sexual initiation, and fighting was especially prevalent among African American males (Weden \& Zabin, 2005).

As discussed PBT, TPB, and SCT share many conceptual similarities and predictors. However, given that PBT is a more comprehensive theory for explaining problem behavior in adolescents, this this study uses PBT as its guiding theoretical framework to examine the cooccurrence of risky behaviors and factors contributing to risky behaviors among adolescents.

In this cross-sectional study, using the Youth Risk Behavior Surveillance System (YRBSS) data, we drew on a theoretical approach informed by the Problem Behavior Theory (PBT) to conceptually guide the variable selection, and hypotheses formulation in investigating the cooccurrence of health risk behaviors among adolescents. As discussed above, PBT includes three systems, personality, behavior, and the perceived environment. In this present research study we will use the behavior system as our guiding approach to addressing our research questions related to the co-occurrence of adolescent risk behaviors. 


\title{
Chapter 3
}

\section{A latent class analysis of the co-occurrence of risk behaviors among adolescents}

\begin{abstract}
Objectives: To examine the co-occurrence of adolescent health risk behaviors inlcuding substance use, behaviors contributing to intentional and unintentional injuries, bullying, risky sexual behavior, depressive symptoms and sucidality, unhealthy weight control, poor dietary habits, and physical inactivity, and sedentary behaviors; and determine whether there are any differences in the pattern of the co-occurrence of these risk behaviors by sex.

Methods: Secondary analysis of the national 2013 Youth Risk Behavior Surveillance System (YRBSS) data was conducted to address the objectives of this study. Our sample included 13,583 high school students. We conducted LCA for the overall sample of adolescents, and separately by sex.

Results: Our analyses for the overall sample yielded five classes of adolescents distinguishable by the probability of their engagement in risky behaviors. The majority of adolescents $(53 \%)$ belonged to the low risk subgroup (class). Class 2 accounted for $15 \%$ of adolescents and over $90 \%$ of adolescents in this subgroup reported not wearing a helmet while riding a bicycle, $40 \%$ reported riding with a driver who had been drinking alcohol, and 63\% reported texting while driving a car or other vehicle. Over $14 \%$ of adolescents belonged to Class 3 and these youth had a higher probability of being depressed and having suicidal thoughts (81\% and $64 \%$, respectively). Class 4 accounted for over $9 \%$ of adolescents. Adolescents in this subgroup reported high probabilities for current cigarette use (97\%), current tobacco use (99\%), and current alcohol use (73\%), and over half of adolescents in this subgroup reported using marijuana (52\%). Class 5 accounted for $8.5 \%$ of adolescents identified as high risk polysubstance users. Adolescents who belonged to this subgroup endorsed most of the risk behaviors including high probabilities of cigarette (79\%), alcohol (93\%), marijuana (81\%), and prescription drug use (77\%). Over half of adolescents in this subgroup engaged in risky sexual activities such as no condom use during last sexual intercourse (56\%), and drinking alcohol or using drugs before last sexual intercourse (56\%). Three distinct subgroups were identified for boys and four subgroups were identified for girls based on their engagement in risky behavior. Both boys and girls endorsed alcohol use, binge drinking, and polysubstance use including tobacco, marijuana, and prescription drug use. Girls had a distinct Class that endorsed higher probabilities of depressive symptoms and suicidal thoughts.

Conclusion: This study found that several risk behaviors among adolescents are interrelated regardless of sex. However, sex differences in the higher probability of certain patterns such as depressive symptoms and suicidality among girls highlight the importance of developing interventions that consider the demographic composition of adolescents.
\end{abstract}

Keywords: risk behavior, problem behavior, Latent Class Analysis, adolescents, YRBSS 


\subsection{Introduction}

By traditional markers such as rates of mortality, chronic disease, and hospitalization, adolescence is a period when youth and young adults are considered generally healthy. Research on the health status of United States' youths has shown that the main adolescent health threats are based on their behavioral choices (Resnick et al., 1997; Brener \& Collins, 1998; Kann et al., 2014) that place them at risk for the leading causes of morbidity and mortality (Kann et al., 2014). For example, tobacco, illicit drugs, and alcohol use, violence, risky sexual behaviors, and poor dietary habits could result in poor health and disability, impacting a healthy and productive adult life.

The leading causes of mortality for adolescents include unintentional injuries (48\%) with vehicular injuries accounting for the majority of these deaths, followed by homicide (13\%), and suicide (11\%) (Minino, 2010). Major causes of morbidity among adolescents include substance use (tobacco, alcohol and other drug use), risky sexual behaviors, and obesity resulting from poor dietary behaviors and physical inactivity (Blum and Qureshi, 2011). In 2013, 22\% of students reported current tobacco use (one or more cigarettes, cigars, snuff, dip, or chew on one or more days of the past 30 days), $34 \%$ of high school students reported being currently sexually active (sexual intercourse with at least one person during the 3 months before the survey) and of those adolescents only $60 \%$ reported that either they or their partner had used a condom during last sexual intercourse (CDC, 2014). Adolescents who engage in unprotected sexual activity have a higher risk for sexually-transmitted infections (STIs) including HIV, and unwanted pregnancy. Recent data indicate an estimated 329,772 births; 548,032 cases of chlamydia, gonorrhea, and syphilis; and 2,240 cases of HIV annually among youth aged 15-19 years (Kann et al., 2014). The negative health consequences of STIs during adolescence include pelvic inflammatory 
disease, infertility, ectopic pregnancy, preterm births, and fetal abnormalities (Advocates for Youth, 2010; Hamilton, Martin, \& Ventura, 2010).

Mental health challenges, such as depression experienced during adolescence also have adverse effects on overall health and development of youth (Costello et al., 2008; Bhatia \& Bhatia, 2007). Recent prevalence rates on adolescent depression indicate that $30 \%$ of students nationwide had felt depressive symptoms (felt so sad or hopeless almost every day for two or more consecutive weeks, that they stopped doing some usual activities during the 12 months) (Kann et al., 2014). In recent years, bullying has been identified as a public health issue in the United States because of its high prevalence and severe consequences. Bullying is linked to many negative outcomes including impacts on mental health, substance use, and suicide (Spriggs et al., 2007; Bannink et al., 2014). Recent national data, show that nearly $20 \%$ of adolescents had been bullied on school property and $15 \%$ of adolescents had been electronically bullied during the 12 months before the YRBSS survey (Kann et al., 2014).

National survey data also indicate that the majority of adolescents in the US do not have diets that meet the recommended dietary guidelines in the US (DHHS, 2015). In addition to poor dietary behavior, sedentary behavior has also emerged as a public health issue among adolescents (Pearson et al., 2011; Owen et al. 2010; DHHS, 2014). Some researchers define "sedentary" as not meeting a criterion level of physical activity (the Healthy People 2020 guidelines recommend at least 60 minutes each day of aerobic physical activity for children and adolescents). However, sedentary behavior also includes excessive sitting time or very low levels of energy expenditure through sitting and laying down (Owen et al., 2010). The Healthy People 2020 physical activity objectives address sedentary behaviors by calling for an increase in the proportion of children and adolescents who do not exceed recommended limits for screen time, 
including viewing television less than three hours a day and using a computer or playing computer games outside of school (for non-school work) less than three hours a day (DHHS, 2010).

\section{Co-occurrence of Health Risk Behaviors (HRBs)}

Health risk behaviors among adolescents are often interrelated, and they extend into adulthood (Hale et al., 2014; Jessor et al. 1995). As early as the 1970s, Richard Jessor and his colleague highlighted the importance of the interrelationships between various risk behaviors, which he called "problem behavior" (Jessor \& Jessor, 1977). Jessor defined problem behavior as any behavior that departs from the social and legal norms of society and causes a social control response from external sources (Jessor \& Jessor, 1977). A number of studies building on the work of Jessor and colleagues have documented a correlation between two or more risk behaviors providing partial evidence of Jessor's "problem behavior" theory (Brener \& Collins, 1998; Khan, Berger, Wells, \& Cleland, 2012; Lindberg, Boggess, \& Williams, 2000; MacArthur et al., 2012; Zweig, Lindberg, \& McGinley, 2001). For example, studies have identified these behavior combinations: aggression, substance use, and suicidal behavior (Garrison, McKeown, Valois, \& Vincent, 1993) substance use, sexual activity, and suicidal behavior (Burge, Felts, Chenier, \& Parrillo, 1995); substance use and violence (Dukarm et al., 1996); and substance use and risky sexual activity (Graves \& Leigh, 1995; Khan et al., 2012; Shrier et al., 1997). In one study, Zweig and colleagues (2001) examined the co-occurrence among eight specific behaviors (sexual activity, general alcohol use, binge drinking, cigarette use, marijuana use, other illicit drug use, fighting, and suicide) using data from the National Longitudinal Study of Adolescent to Adult Health (Add Health). The findings demonstrated that the majority of adolescents in grades 9-12 had low risk profiles. The highest risk profiles indicated links between substance use, 
sexual activity, violence, and suicide and found a link between sexual activity and substance use supporting previous research. In another study using cluster analysis, one cluster was characterized by alcohol use, smoking, marijuana use, and sexual intercourse among white adolescents and young men, and another group of African American adolescents formed a cluster characterized by truancy, early sexual activity, and fighting (Weden \& Zabin, 2005). Drug use and delinquent behaviors have been associated with an increased risk of depressed mood in adolescents (Costello, Swendsen, Rose, \& Dierker, 2008). Prior studies have linked alcohol use to early sexual activity, multiple partners and unprotected sexual intercourse (Fergusson \& Lynskey, 1996; Strachman, Impett, Henson, \& Pentz, 2009).

Although the studies described above contribute to the literature about adolescent risk behaviors and the co-occurrence of risk behaviors among this population, they are limited in two important ways. First, is the limited patterns of risk behaviors studied (e.g., substance use and risky sexual behavior, substance use and violence); and second is the changing culture of adolescence since these studies were conducted over a decade ago may be limiting representativeness of the findings to adolescents today (e.g., the impact of social media on adolescent health risk behavior). Our study addressed these two limitations (limited number of risk behaviors and outdated data) by examining the co-occurrence of over 40 indicators contributing to risky behavior among adolescents using recent nationally representative data (2013 YRBSS) to determine patterns of the interrelationships of these risks among adolescents and provide an update on relationships between risks that may have shifted since past studies. Specifically, the objectives of this study were to examine the frequency and co-occurrence of adolescent substance use (including tobacco, alcohol, marijuana and other illicit drug use including prescription drug use), behaviors contributing to violence and unintentional injuries, 
depressive symptoms and suicidal thoughts, bullying, risky sexual behavior, unhealthy weight control, poor dietary habits, and physical inactivity and sedentary behaviors. This study also aimed to determine whether there were any differnces in the co-occurrence of these risk behaviors by sex.

We used Latent Class Analysis (LCA) to determine the co-occurrence of risky behaviors in our sample and to identify possible patterns of risky behaviors by sex. We examined whether there are distinct subgroups among adolescents who report risky behaviors. A total of 40 items or manifest variables covering multiple domains of problem behavior, were used in the latent class analysis. These items were intended to be as inclusive as possible of potential problem behaviors, including behaviors that reflect risk-taking such as riding in a car with someone who has been drinking alcohol or texting and emailing while driving a vehicle. Thus, the items or manifest variables we used in our study are more extensive than those in previous studies, which tend to focus mainly on one or two items covering one or two domains of problem behavior (e.g., tobacco and alcohol use as measures of substance use). Utilization of a pool of items allows for a fuller examination of the potential relationships between multiple forms of adolescent risk-taking behaviors while developing classes that may be of use in understanding the scope and patterns of these behaviors.

\subsection{Methods}

We used data from the 2013 national Youth Risk Behavior Surveillance System (YRBSS) which uses a three-stage, cluster sample design to obtain a nationally representative sample of U.S. adolescents in grades 9 through 12. YRBSS was developed by the Centers for Disease Control and Prevention (CDC) to monitor six categories of priority health-risk behaviors among youth in grades 9-12: 1) behaviors that contribute to unintentional injuries and violence; 2) tobacco use; 3) alcohol and other drug use; 4) sexual behaviors that contribute to unintended 
pregnancy and sexually transmitted diseases (STDs), including human immunodeficiency virus (HIV) infection; 5) unhealthy dietary behaviors; and 6) physical inactivity. In addition, YRBSS monitors the prevalence of obesity and asthma. The YRBSS uses a stratified cluster design of all public and private high schools in the United States with a sampling method to ensure representativeness for youth attending high schools in the U.S. YRBSS data collection occurs biennially during odd-numbered years and represents a cross section of students attending high school for the given year. Data are collected at school using self-administered anonymous surveys. A weight variable created based on student sex, race/ethnicity, and school grade is applied to each record to adjust for student nonresponse and oversampling of black and Hispanic students. The final overall weights are scaled so that the weighted count of students equals the total sample size and the weighted proportions of students in each grade match national population projections for each survey year. For both the national YRBS and the state surveys, sampled schools, classes, and students who refused to participate were not replaced. For the 2013 national YRBS, the school response rate was $77 \%$, the student response rate was $88 \%$ for an overall response rate of $68 \%$ (Kann et al., 2014). In this study, all variables considered were dichotomized as delineated by the 2013 YRBSS Data Users Manual (YRBSS data users manual, 2013). Dichotmous variables represented the percent of students who reported participating or not participating in a behavior. A behavior was determined as risky based on a positive response to engaging in a behavior one or more times during the past 12 months or past 30 days (Brenner et al., 2003).

\section{Measures}

The primary outcome variables included health risk behaviors and depression, a factor contributing to risky behaviors. Specifically the following outcome variables were measured as 
dichotomous variables: 1) current cigarettes use (smoked cigarettes on 1 or more days in the past 30 days), 2) daily cigarette use, 3) current tobacco use, 4) current marijuana use, 5) current alcohol use, 6) binge drinking, 7) lifetime cocaine use, 8) lifetime glue/aerosol spray/inhalant use, 9) lifetime ecstasy use, 10) lifetime prescription drug use, 11) helmet use, 12) seatbelt use, 13) rode with a driver who had been drinking, 14) drove when drinking alcohol, 15) texted while driving a vehicle, 16) carried a weapon, 17) carried a weapon on school property, 18) were in a physical fight, 19) depressive symptoms, 20) suicidal thoughts, 21) plan to attempt suicide, 22) bullied on school property, 23) electronically bullied, 24) sexual intercourse with 4+ persons (during life), 25) sexual intercourse with at least one person during the last 3 months (currently sexually active), 26) condom use, 27) drank alcohol or used drugs before last sexual intercourse, 28) did not eat for 24+ hours to lose weight or keep from gaining weight, 29) took diet pills, 30) vomited or took laxatives to lose weight, 31) no fruit consumption, 32) no vegetable intake, 33) did not drink milk, 34) did not eat breakfast, 35) drank soda 2+ time/day, 36) no physical activity, 37) watched TV 3+ hours/day, 38) played video games 3+ hours/day, 39) overweight, 40) obese.

Cigarette Use was measured as the percentage of students who smoked one or more cigarettes on any day of the past 30 days.

Daily Cigarette Use was measured as the percentage of students who ever smoked cigarettes daily, that is, at least one cigarette every day for 30 days.

Current Tobacco Use was measured as the percentage of students who smoked cigarettes or cigars or used chewing tobacco, snuff, or dip on one or more of the past 30 days. Marijuana use was measured as the percentage of students who used marijuana one or more times during the past 30 days. 
Current Alcohol Use was measured as the percentage of students who drank alcohol one or more times during the past 30 days.

Binge Drinking was measured as percentage of students who had five or more drinks in a row, that is, within a couple of hours, during the past 30 days.

Other Drug Use was assessed based on the percentage of students who used any form of cocaine, including powder, crack, or freebase one or more times during their life; the percentage of students who sniffed glue, breathed the contents of aerosol spray cans, or inhaled any paint or sprays to get high one or more times during their life; percentage of students who used ecstasy one or more times during their life; and the percentage of students who have taken a prescription drug (such as OxyContin, Percocet, Vicodin, codeine, Adderall, Ritalin, or Xanax) without a doctor's prescription one or more times during their life.

Behaviors that contribute to unintentional injuries were assessed by the following indicators: Among students who rode a bicycle during the past 12 months, the percentage who never or rarely wore a helmet; percentage of students who never or rarely wore a seatbelt while riding in a car driven by someone else; percentage of students who rode one or more times in a car or other vehicle driven by someone who had been drinking alcohol; among students who drove a car or other vehicle during the past 30 days, the percentage who drove when they had been drinking alcohol one or more times during the past 30 days; among students who drove a car or other vehicle during the past 30 days, the percentage who texted or e-mailed while driving on one or more days during the past 30 days;

Violence related behaviors were assessed by the following indicators: percentage of students who carried a weapon such as a gun, knife, or club on one or more of the past 30 days; percentage of students who carried a weapon such as a gun, knife, or club on school property on 
one or more of the past 30 days; percentage of students who were in a physical fight one or more times during the past 12 months.

Depression was measured as percentage of students felt so sad or hopeless almost every day for two weeks of more in a row that you stopped doing some usual activities during the past 12 months. Suicidal thoughts and attempts were measured as the pecentage of students who seriously consider attempting suicide during the past 12 months; percentgage of students who made a plan about how they would attempt suicide during the past 12 months. Bullying (including cyberbullying) is defined as one or more students tease, threaten, spread rumors about, hit, shove, or hurt another student over and over again. Bullying was assessed based on the percentage of students who had ever been bullied on school property during the past 12 months; and percentage of students who had ever been electronically bullied during the past 12 months (counting being bullied through e-mail, chat rooms, instant messaging, websites, or texting).

Risky sexual behavior was measured based on the percentage of student who had sexual intercourse with four or more people during their life; percentage of students who had sexual intercourse with one or more people during the past three months; the percentage who drank alcohol or used drugs before last sexual intercourse; and condom during last sexual intercourse Unhealthy weight control was assessed based on the percentage of students who went without eating for 24 hours or more (also called fasting) to lose weight or to keep from gaining weight during the past 30 days; percentage of student who took any diet pills, powders, or liquids without a doctor's advice to lose weight or to keep from gaining weight during the past 30 days; and the percentage of students who vomitted or took laxatives to lose weight or to keep from gaining weight during the past 30 days. 
Poor dietary habits were assessed based on YRBSS constructed dichotomous variables that indicate the percentage of students who ate or drank $100 \%$ fruit juices 0 times per day during the past 7 days; the percentage of students who ate vegetables 0 times per day during the past 7 days; the percentage of students who drank a can, bottle, or glass of soda or pop two or more times per day during the past 7 days; the percentage of students who drank 0 glasses per day of milk during the past 7 days; and the percentage of students who ate breakfast on none of the past 7 days. Physical inactivity was assessed based on YRBSS constructed dichotomous variables that indicate the percentage of students who were physically active for a total of 60 minutes on 0 of the past 7 days.

Sedentary behavior was assessed based on YRBSS constructed dichotomous variables that indicate the percentage of students who watched three or more hours per day of TV on an average school day; and the percentage of students who played video games or used a computer for something that was not school work three or more hours per day on an average school day. We also included two variables, overweight and obese, as measures of weight status in our analyses. The YRBSS used age, sex, and BMI to determine weight status (i.e. overweight and obese). When the student had a BMI at or above the $85^{\text {th }}$ percentile and below the $95^{\text {th }}$ percentile then the student was considered overweight. When the student BMI was at or above the $95^{\text {th }}$ percentile then the student was considered obese. If BMI was missing then variables overweight and obesity were set to missing as well (YRBSS, 2013).

Our demographic variable included in the LCA was self-reported measure of either male or female measured as a dichotomous variable based on the YRBSS question "What is your sex?" The descriptive statistics included information on age, grade level, and race/ethnicity in addition to sex. Chronological age was calculated using the participants' self-reported age at the time the 
YRBSS was conducted. Grade level is also a self-reported measure of the current grade level at the time the survey was completed and it was measured as an ordinal variable $\left(9^{\text {th }}\right.$ grade, $10^{\text {th }}$ grade, $11^{\text {th }}$ grade, and $12^{\text {th }}$ grade) based on the YRBSS question "In what grade are you?"

Racelethnicity was measured as a self-reported nominal variable based on two question in the YRBSS, "Are you Hispanic? Yes/No" and "What is your race? American Indian or Alaska Native; Asian; Black or African American; Native Hawaiian or Other Pacific Islander; and White." We grouped race/ethnicity into five cateogries of white, black, Hispanic, multipleHispanic, and other (American Indian, Pacific Islander/Hawaiian Native, Asian, and multiple non-Hispanic).

\section{Statistical Analysis}

The statistical package SAS v. 9.4 (SAS Institute, Inc, Cary, NC) was used for data management and descriptive analyses, to account for the complex survey design of YRBS. Descriptive statistics including frequencies and percentages, were generated using the SURVEYFREQ procedure in SAS to determine the sample demographic and behavioral characteristics.

The overall model structure was determined by fitting an unrestricted latent class model (LCM) to 40 manifest indicators of risky behavior. Consistent with previous literature, we began with a two class model and continued adding additional classes until the best fitting model was obtained (Nylund, Asparouhov, \& Muthén, 2007; Weden \& Zabin, 2005; Pena et al., 2012). This process is used if there are no a priori assumptions about the clustering between the manifest variables, which was the case for this study (Nylund, Asparouhov, \& Muthén, 2007). Fit indices were the likelihood ratio test statistic (LRT; TECH 11- TVuong-Lo-Mendell-Rubin LRT test), 3 information criteria: AIC, the Akaike information criterion; ABIC, adjusted AIC; and BIC, the 
Bayesian information criterion. The latent class probabilities and conditional probabilites used the distribution of the total population across the latent classes and the characteristics of the latent classes (i.e., class membership for specific indicators), and entropy. Mplus v. 7.31 was used to conduct LCA for this study. Mplus accounts for the stratified cluster design of the YRBSS in the analysis by using the "Type = Complex" command (Muthén \& Muthén, 2008). Analyses were adjusted using sample weights of YRBSS to make results generalizable to high school students in the US.

\section{Sample Characteristics}

\subsection{Results}

The sample included 13,583 US high school adolescents. More than half identified as White (55\%); half of the sample was female and the majority were between the ages of 15 and 18 years (Table 1). Table 2 describes the distribution of individual risk behaviors among adolescents in our sample.

Our results indicate that many adolescents are engaged in risky behaviors. Substance use is prevalent among this population. Overall almost $16 \%$ of adolescents reported being current smokers (smoking one or more cigarettes on one or more days of the past 30 days), $32 \%$ had drank alcohol on one or more days of the past 30 days and almost a quarter of youth had used marijuana one or more days of the past 30 days. Prescription drug use was also prevalent among this population, almost $18 \%$ of adolescents had used prescription drugs without a doctor's prescription in the past 12 months. As illustrated in Table 2, adolescents in our sample exhibited behaviors that lead to unintentional injuries and violence. Over a quarter of the adolescents reported texting while driving a car on one or more days of the past 30 days. Many students also engaged in risky sexual activity. Results showed that $32 \%$ of the adolescents in our sample were currently sexually active (i.e., had sexual intercourse with one or more people 
during the 3 months before the survey). Among those adolescents who were currently sexually active, $13 \%$ had not used a condom during their last sexual intercourse.

\section{Description of the Subgroups (Classes)}

Tables 3 and 4 illustrate the fit indices for each class considered in this analysis. We ran latent class analyses beginning at a two-class solution and adding more classes until we saw a plateau in the fit indices. A five-class model provided the best overall fit to the data for risk behaviors among adolescents in our sample. This was determined based on the review of BIC (the lower the BIC the better), the p-value for LRT (the higher the p-value the better), and the value of entropy. Entropy is the measure of classification accuracy and it is defined on $[0,1]$, with values near one indicating high certainty in classification and values near zero indicating low certainly in classification.

As illustrated by Table 5, for the overall sample of adolescents 5 classes (subgroup) of risky behavior were identified. Class 1 accounted for the majority of adolescents (53\%), Class 2 accounted for $15 \%$ of teens, Class 3 accounted for $14 \%$ of adolescents, and classes 4 and 5 comprised of $9.4 \%$ and $8.5 \%$ of adolescents, respectively. Classes 1 and 2 comprised of adolescents who did not engage in risky behaviors, therefore we identified adolescents in these Classes as low risk. Class 3 comprised of adolescents that had a higher probability of being depressed and suicidal than either classes 1, 2 or 4 . Over $80 \%$ of adolescents in this Class reported being depressed, $64 \%$ had suicidal thoughts in the past 12 months, and over 50\% had planned to attempt suicide. Class 4 comprised of adolescents who were tobacco and alcohol users. These adolescents had a high probably (0.97) of being current smokers (had at least 1 or more cigarettes on one or more of the past 30 days), current tobacco users (0.98) (including cigarettes, cigars, snuff, and dip), and current drinkers (at least one or more drinks on one or 
more of the past 30 days) (0.73), compared to the other classes. Class 5 consisted of high risk adolescent who had a high probability of engaging in the majority of risk behaviors including being depressed. These adolescents were identified as high risk polysubstance users. In addition to high probabilities of tobacco, marijuana, and alcohol use, these adolescents also had a high probability of using prescription drugs such as OxyContin, Percocet, Vicodin, Codeine, Adderall, Ritalin, or Xanax. Probability of physical fights in the past 12 months was moderate for the adolescents in this subgroup.

\section{Description of Subgroups by Sex}

Based on the fit statistics, we determined a 4-Class model for girls and a 3-Class model for boys in our sample (see Table 4). The majority of adolescents in both groups (girls and boys) belonged, to the low risk subgroup (50\% of girls, and 62\% of boys) or class 1 .

Among boys Class 2 accounted for over a quarter (26\%) of adolescents. The youth in this subgroup were identified as 'Alcohol Drinkers' because of their high probability of current alcohol use (0.77). Class 3 among boys accounted for $12 \%$ of the sample and this subgroup of boys were identified as 'High-risk Polysubstance Users.' All members of this class exhibited a higher probability of use for cigarettes, tobacco, alcohol, and binge drinking compared to classes 1 and 2. In addition, boys in this subgroup had a high probability of life time prescription drug use compared to classes 1 and 2. About two-thirds of boys in Class 3 indicated carrying a weapon such as a gun, knife, or club during the past 30 days. Boys in this group also had a high probability of being involved in a physical fight one or more times during the past 12 months.

Almost $50 \%$ of girls in our sample belonged to Class 1 - low risk subgroup. Class 2 accounted for $20 \%$ of girls and members of this class endorsed alcohol use and about half of the girls in this subgroup reported binge drinking (five or more drinks of alcohol in row, within 
couple of hours, on one or more days of the past 30 days). Girls in this subgroup were identified as 'Alcohol Drinkers.' Approximately 19\% of girls in our sample belonged to Class 3. Most of the girls in this subgroup reported having depressive symptoms (0.84), and suicidal thoughts (0.68), and over half of them reported making a plan to attempt suicide (0.52) in the past 12 months. We identified girls in this subgroup as 'Depressed and Suicidal.' These girls also had a higher probability of being bullied compared to girls in class 1 and $2(0.48,0.24$, and 0.15 , respectively). Class 4 accounted for almost $12 \%$ of girls in our sample. Girls in this subgroup endorsed the majority of risk behaviors examined in our study and were identified as 'High Risk - Polysubstance Users.' Most girls in this subgroup endorsed substance use behavior. Eighty percent of girls reported being current cigarette smokers, $74 \%$ were current marijuana users, and $88 \%$ were alcohol drinkers, with $71 \%$ reporting binge drinking (five or more drinks in a row). These girls also endorsed drug use, especially prescription drug use (68\%). Girls in this subgroup also reported having depressive symptoms and suicidal thoughts. In addition, 79\% of girls in this subgroup reported being sexually active and of those who were sexually active $61 \%$ reported not using a condom during their last sexual intercourse, and $44 \%$ reported drinking alcohol or using drugs before their last sexual intercourse.

\subsection{Discussion}

Latent class analysis for the overall sample of adolescents in this current study point to five subgroups of youth engaging in risky behavior: those not engaging in any risk behavior low risk (Classes 1 and 2), those with depressive symptoms and suicidal thoughts (Class 3), those with high probability of tobacco and alcohol use (Class 4), and those identified as polysubstance users (Class 5). Similar to previous research our study found that the majority of high school adolescents belonged to the low risk subgroup (Zweig et al., 2001; Pena et al., 2012; 
Connell et al., 2009) and this was true for both boys and girls (62\% for boys and 50\% for girls). The highest risk profiles for both boys and girls accounted for the lowest percentage of adolescents (12.2\% for boys and $11.6 \%$ for girls). Supporting previous research which linked two or three risk behaviors (Zweig et al., 2001; Durkham et al., 1996; Shrier et al., 1996; Burge et al., 1995) we found that adolescents in the highest-risk subgroups engaged in multiple risk behaviors including substance use, risky sexual behavior, and behaviors that lead to unintentional injuries and violence.

For both boys and girls, the polysubstance use-high-risk subgroup provides not only more evidence of the interrelationship between substance use and risky sexual behavior, but also the links between different types of substances and engaging in behaviors that lead to unintentional injury and violence such as texting and driving, and riding with a drunk driver, and getting into physical fights. Despite the similarities in subgroups of risky behavior for boys and girls, there were sex differences in the levels and types of risky behavior among adolescents in general. When analyses were stratified by sex, girls had higher probabilities of depressive symptoms, suicidal thoughts, and plans to attempt suicide, and engaging in sedentary behavior (playing video games three or more hours a day) than boys. Whereas girls in the polysubstance use subgroup reported high rates of depressive symptoms and suicidality along with the majority of other risk behaviors, boys in the polysubstance use subgroup reported relatively low depressive symptoms and suicidal thoughts and attempts. This finding contradicts past research, finding a distinct risk profile with high rates of marijuana use and suicidal behavior among boys (Zweig et al., 2001). In our study, no significant differences were found between boys and girls in terms of the prevalence of substance use, however boys did report higher rates of weapon carrying and getting in physical fights than girls. 


\section{Strengths and Limitations}

This study has several strengths, including the use of a dataset that is nationally representative of the high school adolescents in the U.S.; and the inclusion of multiple measures of particular risk behaviors. For example, for substance use we measured current tobacco use, current cigarette use, alcohol use, binge drinking, marijuana use, and prescription drug use. This is a strength because it allows for a fuller examination of the potential relationships between multiple forms of adolescent risk-taking behaviors while developing classes that may be of use in understanding the scope and patterns of these behaviors.

Another strength of the study is presenting recent data on adolescent risk behaviors. Previous studies using data collected more than a decade ago may not fully represent the risk behaviors adolescents engage in today. For example, the use of social media has become an integral part of the daily life of youth in the past decade. While the use of social media may have some benefits for adolescent health and development such as extending friendships, serving a source of information and advice on health concerns, and allowing adolescents who are marginalized because of their sexuality, or disability to seek and find support networks, it also provides more opportunities for health risk behavior such as cyberbullying and sexting. Furthermore, youth today through access to media such as video and computer games have more opportunities for engaging in sedentary behavior than did their previous cohort. In addition, a strength of the study is the use of LCA to determine the co-occurrence of risky behaviors and to examine whether there are distinct subgroups among adolescents who report risky behaviors by treating each risk behavior as separate but related domains of potentially risky adolescent behavior. By using this approach, we were able to examine how patterns of certain behaviors 
influence involvement in distinct patterns of other risky behaviors while also incorporating sex as a covariate.

Although our study is based on a nationally representative sample of high school adolescents in the U.S. and it contributes to the knowledge about adolescent risk behaviors, it does have a number of limitations. YRBSS data are cross-sectional, therefore, causality cannot be established. In addition, despite previous studies suggesting that YRBSS survey questionnaire items have produced valid responses and have high degree of reliability (Brener et al., 2002), the survey uses self-reported measures of behavior which may not be accurate especially given the sensitive nature of some of the items used in this study. Our results are generalizable only to adolescents who attend school, limiting our ability to generalize findings to adolescents who are not enrolled in school or who may have dropped out of school. Furthermore, our research lacks important contextual factors that may influence adolescents' propensity to engage in certain risk behaviors or combination of risk behaviors. Although our study was guided by a construct of problem behavior theory, it was limited to one construct of the theory, the behavior system, based on the scope of our research and availability of the variables related to adolescent risk behavior in the YRBSS. Future research investigating the influence of important contextual factors such as family structure, peer relationships, self-esteem, and socioeconomic, and parental education, using multiple data sources is warranted.

Despite these limitations, our study provides evidence to the interrelationships between risky behaviors in adolescents. To our knowledge ours is the first study to have examined the cooccurrence of 40 manifest variables accounting as elements or indicators of different risk behaviors using a nationally representative sample of high school adolescents in the U.S. 


\section{Implications and Future Research}

The evidence provided by our study, consonant with problem behavior theory, indicates that risk behaviors co-occur may have important implications for prevention, intervention, and health promotion efforts. Specifically, these behavior change efforts need to recognize health risk behaviors in this population as interrelated rather than as isolated and unrelated actions. Most existing interventions or programs focus on one or two primary correlates that are believed to be responsible or contribute to problem behavior among adolescents and these programs often provide a simple or one dimensional approach to addressing and preventing risk behaviors in adolescents. For example, the Scared Straight is a violence-prevention program with the premise that youth need to have a better appreciation of the negative consequences of behavior and or an attitude change. This program targets juvenile delinquents or youth at risk of becoming delinquent and its main goal is to deter youth from future criminal behavior. The program components include prison tours and presentations by inmates, giving youth an idea of the harsh realities of life in prison and the consequences of their violent/criminal behavior (Klenowski et al., 2010). This is an oversimplified program that makes the assumption that a particular negative behavior is caused by a single determinant and youth just need to be "Scared Straight" by observing the consequences of such negative behavior. Although studies have found that those who participate in Scared Straight-types programs are more likely to commit crimes in the future (Pertrosino, Turpin-Petrosino, Hollis-Peel, \& Lavenberg, 2013; Petrosino, Turpin-Petrosino, \& Buehler, 2003), the program is still widely used across the country because of concerns about public safety.

Interventions may be more successful and make a greater impact if they consider psychosocial and environmental contexts in which adolescent make decisions. Integrated models 
of preventing multiple risk behaviors are warranted to not only provide accurate information and teach useful life-skills for handling pressure and conflict, but also timed properly to the developmental level of adolescents, and strategically delivered taking into account demographic factors. A study examining the influence of physical activity on smoking cessation among youth found that implementing a multi-behavior program that addressed both smoking and physical activity in high school adolescents was significantly more effective than a brief intervention. Horn and colleagues randomly assigned high schools to one of three study conditions, brief intervention (BI), Not on Tobacco $(\mathrm{N}-\mathrm{O}-\mathrm{T})$ a proven teen cessation program, or N-O-T + FIT which included a physical activity module. The results showed that adding the physical activity component to N-O-T was particularly successful in smoking cessation among boys (Horn et al., 2013).

Further research is needed to examine health risk behaviors using nationally representative longitudinal data that include contextual variables, considering all three elements of the problem behavior theory, to identify risk and protective factors leading to behavioral choices in the adolescent population. Our study findings suggest that future research should focus on sex differences in health risk behaviors among adolescents. The co-occurrence of depression and polysubstance use behavior was stronger and more prevalent for girls than for boys in our study. A study by Connell et al (2009) found that association between substance use and either low or high frequency multipartner sexual activity was significantly stronger for girls than it was for boys. This study elaborated that although girls are less likely to be identified in either low or high multipartner sexual risk groups than boys, their relative risk for involvement in these behavior increases when they engage in frequent polysubstance use behavior. An understanding of how one's social and physical environment could affect behavioral decision- 
making could be an important element to consider in the development of effective prevention and intervention efforts, and policies in respect to adolescent health. 


\title{
Chapter 4
}

\section{Overweight and obesity and health risk behaviors among high school adolescents in the United States}

\begin{abstract}
Objective: The purpose of this study was to examine the association between overweight and obesity and risky behaviors including substance use (tobacco, alcohol, marijuana, and illicit drug use), behaviors contributing to violence and unintentional injuries, depressive symptoms (as a risk factor), suicidal thoughts/attempts, bullying, risky sexual behaviors, unhealthy weight control, poor dietary habits, physical inactivity and sedentary behaviors.

Methods: Secondary data analysis was conducted using national data from 2013 Youth Risk Behavior Surveillance System (YRBSS). The primary outcome variables were cigarrette use, alcohol, marijuana, and other illicit drug use, behaviors contributing to unintentional injuries, behaviors contributing to violence, bullying, depression, suicidal thoughts and attempts, risky sexual behaviors that contribute to unintended pregnancy and sexually transmitted infections, unhealthy weight control, poor dietary habits, physical inactivity, and sedentary behaviors. The study population included 13,583 high school students. Odds ratios and adjusted odds ratios were calculated using logistic regression. All analyses were conducted using SAS 9.4.

Results: Results showed that obese students were significantly more likely to be current cigarettes smokers ( $\mathrm{AOR}=1.44,95 \% \mathrm{CI}, 1.15-1.79)$, be depressed (AOR=1.19, 95\% CI, 1.041.37), have suicidal thoughts $(\mathrm{AOR}=1.51,95 \% \mathrm{CI}, 1.31-1.76)$, have planned a suicide $(\mathrm{AOR}=1.61,95 \% \mathrm{CI}, 1.35-1.92)$, be bullied at school and electronically bullied (AOR=1.47, 95\% CI, 1.28-1.68 and $\mathrm{AOR}=1.20,95 \% \mathrm{CI}, 1.00-1.45)$, engage in behaviors contributing to violence $(\mathrm{AOR}=1.39,95 \% \mathrm{CI}, 1.24-1.57)$, have poor dietary habits $(\mathrm{AOR}=1.37,95 \% \mathrm{CI}, 1.20$ $1.55)$ and engage in sedentary behavior $(\mathrm{AOR}=1.74 ; 95 \% \mathrm{CI}, 1.52-1.99)$ compared to normal weight adolescents. Both overweight and obese adolescents were significantly more likely to engage in unhealthy weight control $(\mathrm{AOR}=3.26,95 \% \mathrm{CI}, 2.77-3.84$ and $\mathrm{AOR}=7.56,95 \% \mathrm{CI}$, 6.33-9.04, respectively) compared to normal weight adolescents. Results also showed that among students, obese students were significantly less likely to engage in behaviors that lead to unintentional injury $(\mathrm{AOR}=0.83,95 \% \mathrm{CI}, 0.73-0.95)$, and engage in risky sexual behavior $(\mathrm{AOR}=0.86,95 \% \mathrm{CI}, 0.75-0.98)$ compared to their normal weight peers.

Conclusions: Obesity was positively associated with cigarette use, engaging in behaviors that lead to violence, being depressed, suicidal, bullied, engage in unhealthy weight control, and sedentary behavior. There is an inverse relationship between obesity and engaging in behaviors that lead to unintentioanl injury, and risky sexual behavior.
\end{abstract}

Keywords: overweight, obesity, adolescence, risky behaviors, YRBSS 


\subsection{Introduction}

Overweight and obesity have become an increasingly prevalent public health issue in the past few decades, affecting all age groups, and race/ethnicities in the United States. In recent years, children and adolescents have experienced a dramatic increase in overweight and obesity rates. Between 1986 and 1998 overweight and obesity among children and adolescents increased by more than $120 \%$ for black and Hispanic children and adolescents, and by $50 \%$ for whites (Strauss and Pollack 2001). In the past few generations, childhood obesity has more than doubled in children and quadrupled in adolescents (Ogden, Carroll, Curtin et al., 2014). Nationally, $32 \%$ of children aged 2-19 years old are overweight or obese (Ogden et al., 2014).

The immediate health effects of overweight and obesity in youth include risk factors for cardiovascular disease, such as high cholesterol or high blood pressure (Freedman et al., 2005; Ogden et al., 2014). In addition, obese children and adolescents are more likely to have prediabetes, a condition in which blood glucose levels indicate a high risk for development of diabetes (Baker, Olsen, \& Sørensen, 2007; Ogden et al., 2014). Children and adolescents who are overweight and obese are at much greater risk for bone and joint problems, sleep apnea, and social and psychological problems such as poor self-esteem and stigmatization (Ogden et al., 2014).

Obesity in childhood and adolescence is likely to track into adulthood (Reilly et al., 2003; Singh, Mulder, Twisk, Van Mechelen, \& Chinapaw, 2008) and has multiple negative long-term outcomes (Anuradha et al., 2015; Reilly et al., 2003; Singh et al., 2008). Early literature reviews (Must et al., 1999) and more recent systematic reviews of the literature (Park, Falconer, Viner, \& Kinra, 2012; Reilly \& Kelly, 2011) suggest increased risk of cardio-metabolic morbidity (e.g., diabetes, stroke, coronary heart disease, hypertension) and all-cause mortality in adulthood 
among those who were overweight or obese during childhood (Baker et al., 2007). Although complications and adverse health effects of adolescent overweight and obesity are well documented and include metabolic health risk, chronic diseases, and psychosocial issues (Ebbeling, Pawlak, \& Ludwig, 2002; Ludwig, 2007), much less is known about the association of overweight and obesity with other health-risk behaviors during the teen years. Overweight and obese adolescents are particularly vulnerable to risky behavior compared to their healthy weight peers, because overweight and obese youth are more likely to experience stigmatization (Farhat, Iannotti, \& Simons-Morton, 2010). In addition to the normative challenges of adolescence, the stress of managing an unhealthy weight may make these adolescents more susceptible to engaging in risky behaviors (Booker, Gallaher, Unger, Ritt-Olson et al., 2004; Farhat et al., 2010).

A number of studies have examined the effects of overweight and obesity on a limited number of adolescent risky behaviors. Studies that have explored this association have shown a positive relationship between Body Mass Index (BMI) and smoking initiation (Lowery et al., 2002), substance use including alcohol, tobacco and drug use (Pasch et al., 2008), risky sexual behavior (Lowery et al., 2014; Everett et al., 2013; Gordon et al., 2016), fighting (Zeller et al., 2008), and bullying (Zeller et al., 2008; Janssen et al., 2004; Griffiths et al., 2006).

Research showed that the initiation of smoking is more likely among overweight adolescents compared to their healthy weight peers and this behavior has been associated with weight loss aspirations among overweight adolescents (Cawley, Markowitz, \& Tauras, 2004). Drinking alcohol is also associated with overweight obesity. Research shows that those drinking heavily during adolescence are nearly four times more likely to be overweight or obese as adults (Oesterle, Hill, Hawkins, et al., 2004). 
Studies exploring the association of overweight obesity and sexual behavior among adolescents found that among sexually active high school students, overweight and obese female adolescents were more likely than the healthy weight female adolescents to engage in risky sexual behavior (i.e. have multiple sexual partners, have sex without a condom) (Lowery et al., 2014; Gordon et al., 2016). These studies found that overweight or obese teenage girls are more likely to have ever had anal sex compared to their healthy weight peers (Everett et al., 2013; Gordon et al., 2016).

Research shows that body dissatisfaction, especially among female adolescents, has a significant effect on the likelihood of suicidal behaviors. A study by Dave and Rashad (2009) found that any perception of being overweight increased the probability of suicidal thoughts by 5.6 percent, and the probability of suicide attempts by 3.2 percent.

Although previous studies have explored the association of selective risk behaviors and overweight obesity among adolescents, additional research is needed to provide a comprehensive understanding of the relationships between overweight obesity and health risk behaviors among adolescents. The purpose of this current study was to examine the association between overweight and obesity and risky behaviors and factors contributing to risky behaviors including depression, suicidal thoughts/attempts, substance use (tobacco, alcohol, marijuana, and illicit drug use), behaviors contributing to violence and unintentional injuries, bullying, risky sexual behaviors, unhealthy weight control, poor dietary habits, physical inactivity and sedentary behaviors. We also explored whether there were any differences in engaging in risky behaviors based on whether the adolescent was overweight or obese. 


\subsection{Methods}

Secondary data analysis was conducted using national data from 2013 Youth Risk Behavior Surveillance System (YRBSS). YRBSS administers a biennial school-based survey Youth Risk Behavior Survey (YRBS) that uses a three-stage, cluster sample design to obtain a nationally representative sample of U.S. high school adolescents in grades 9-12. YRBSS is conducted by the CDC to monitor health risk behaviors among high school students in the U.S.

A weight variable created by the YRBSS based on student sex, race/ethnicity, and school grade was applied to each record to adjust for student nonresponse and oversampling of black and Hispanic students. The 2013 YRBSS standard questionnaire included 23 questions related to unintentional injuries and violence; 10 questions on tobacco use; 18 questions on alcohol and other drug use; seven questions on sexual behaviors; 16 questions on body weight and dietary behaviors, including height and weight; five questions on physical activity; and two on other health-related topics (i.e., asthma and sleep). YRBSS also monitors the prevalence of obesity among adolescents. Additional information about the YRBSS methods and sampling have previously been described (Kann et al., 2014).

\section{Measures}

The primary outcome variables of this study included health risk behaviors and depression, as a factor contributing to risky behaviors. Specifically, the following outcome variables were measured as dichotomous variables: cigarrette use, alcohol use, marijuana use, and other illicit drug use, behaviors contributing to unintentional injuries, behaviors contributing to violence, bullying, depression, suicidal thoughts/attempts, risky sexual behaviors (i.e. behaviors that contribute to unintended pregnancy and sexually transmitted infections), unhealthy weight control, poor dietary habits, physical inactivity, and sedentary behaviors. All 
variables considered were dichotomized as delineated by the 2013 YRBSS Data Users Manual (YRBSS data users manual, 2013). Dichotmous variables represent the percent of students who report participating or not participating in a behavior. A behavior was determined as risky based on a positive response to engaging in a behavior one or more times during the past 12 months or past 30 days (Brenner et al., 2004; Ratcliff et al., 2011).

Cigarette Use was measured based on the question "During the past 30 days, on how many days did you smoke cigarettes?" a response of one or more days was measured as current cigarette use.

Alcohol Use was assessed based on the question "During the past 30 days, on how many days did you have at least one drink of alcohol?" a response of one or more days was coded as current alcohol use.

Marijuana use was assessed based on the question "During the past 30 days, how many times did you use marijuana?" A response of one or more times was coded as current marijuana use. Behaviors that contribute to unintentional injuries were assessed by the questions "When you rode a bicycle during the past 12 months, how often did you wear a helmet?" "How often do you wear a seat belt when riding in a car driven by someone else?" a response of rarely/never was coded as risky behavior. "During the past 30 days, how many times did you ride in a car or other vehicle driven by someone who had been drinking alcohol?" "During the past 30 days, how many times did you drive a car or other vehicle when you had been drinking alcohol" a response of one or more times was coded as a risky behavior, and "During the past 30 days, on how many days did you text or e-email while driving a car or other vehicle?" a response of one or more times was coded as a risky behavior. A new dichotomous variable named unintentional injury 
was formed that coded participation in risky behavior as 1 and not participating in any risky behavior related to unintentional injury as 2 .

Violence related behaviors were assessed by the questions "During the past 30 days, on how many days did you carry a weapon such as a gun, knife, or club?” “..., on how many days did you carry a weapon such as a gun, knife, or club on school property?" a response of one or more days was coded as a risky behavior, and "During the past 12 months, how many times were you in a physical fight?" a response of one or more times was coded as a risky behavior.

Suicidal thoughts and attempts were measured based on the questions, "During the past 12 months, did you ever seriously consider attempting suicide?” and “...., did you make a plan about how you would attempt suicide?" Responses of yes to the questions were coded as risky behavior.

Depression was measured as a dichotomous variable based on the question "During the past 12 months, did you ever feel so sad or hopeless almost every day for two weeks of more in a row that you stopped doing some usual activities?"

Bullying (including cyberbullying) is defined as one or more students tease, threaten, spread rumors about, hit, shove, or hurt another student over and over again. Bullying was assessed based on two questions, "During the past 12 months, have you ever been bullied on school property?” and “..., have you ever been electronically bullied? (Count being bullied through email, chat rooms, instant messaging, websites, or texting.) A response of yes was marked as being engaged in risky behavior.

Sexual behavior was measured based on the questions "During the past 3 months, with how many people did you have sexual intercourse?" a response of one or more was marked as currently sexually active, "The last time you had sexual intercourse, did you or your partner use a 
condom?" response of no was marked as no condom use, and "During your life, with how many people have you had sexual intercourse?" a response of four or more sexual partners was marked as risky sexual behavior, and "Did you use alcohol or use drugs before you had sexual intercourse last time?"a response of yes was marked as risky behavior. A variable called risky sexual behavior was formed based on responses of yes to any of the above four questions.

Unhealthy weight control was assessed based on the questions "During the past 30 days, did you go without eating for 24 hours or more (also called fasting) to lose weight or to keep from gaining weight?” “...., did you take any diet pills, powders, or liquids without a doctor's advice to lose weight or to keep from gaining weight?" “...., did you vomit or take laxatives to lose weight or to keep from gaining weight?" Responses of yes to any of the questions above were marked as unhealthy behavior.

Poor dietary habits were assessed based on YRBSS constructed dichotomous variables that indicate "the percentage of students who ate or drank $100 \%$ fruit juices 0 times per day during the past 7 days," "the percentage of students who ate vegetables 0 times per day during the past 7 days," “...who drank a can, bottle, or glass of soda or pop two or more times per day during the past 7 days," ".... who drank 0 glasses per day of milk during the past 7 days," and " the percentage of students who ate breakfast on none of the past 7 days." A new variable called poor dietary habits was formed based on a response of yes to any of the above questions.

Physical inactivity and sedentary behavior were assessed based on YRBSS constructed dichotomous variables that indicate "the percentage of students who were physically active for a total of 60 minutes on 0 of the past 7 days," "the percentage of students who watched three or more hours per day of TV on an average school day," "percentage of students who played video 
games or used a computer for something that was not school work three or more hours per day on an average school day."

We used two YRBSS variables, Overweight, and Obese, as our main predictor variables. The YRBSS collected height and weight information based on two questions "how tall are you without your shoes on?" and "how much do you weigh without your shoes on?" If height was missing then weight was set to missing and vice versa, also if either feet or inches was not filled in or was not readable then height was set to missing. Body Mass Index (BMI) was calculated using the formula

$$
B M I=k g / m 2=\text { Weight }(\text { in } \mathrm{kg}) /[\text { Height }(\text { in } \mathrm{m}) 2]
$$

The YRBSS used age, Sex, and BMI to determine weight status (i.e. overweight and obese). When the student had a BMI at or above the $85^{\text {th }}$ percentile and below the $95^{\text {th }}$ percentile then the student was considered overweight. When the student BMI was at or above the $95^{\text {th }}$ percentile then the student was considered obese. If BMI was missing then variables overweight and obesity were set to missing as well (YRBSS, 2013).

Demographic variables included, age, sex, grade level, and race/ethnicity. Chronological age was calculated using the participants' self-reported age at the time the YRBS was conducted. Sex was a self-reported measure of either male or female and was measured as a dichotomous variable based on the YRBSS question "What is your sex?"

Grade level is also a self-reported measure of the current grade level at the time the survey was completed and it was measured as an ordinal variable $\left(9^{\text {th }}\right.$ grade, $10^{\text {th }}$ grade, $11^{\text {th }}$ grade, and $12^{\text {th }}$ grade) based on the YRBSS question "In what grade are you?"

Racelethnicity was measured as a self-reported nominal variable based on two question in the YRBSS, "Are you Hispanic? Yes/No" and "What is your race? American Indian or Alaska 
Native; Asian; Black or African American; Native Hawaiian or Other Pacific Islander; and White." We grouped race/ethnicity into five cateogries of white, black, Hispanic, multipleHispanic, and other (American Indian, Pacific Islander/Hawaiian Native, Asian, and multiple non-Hispanic).

\section{Statistical Analysis}

The statistical package SAS v. 9.4 (SAS Institute, Inc, Cary, NC) was used for data management and analyses, and to account for the complex survey design of YRBS. Descriptive statistics including frequencies and percentages, using the SURVEYFREQ procedure in SAS, were used to determine the sample demographic and behavioral characteristics. Multivariable logistic regression analysis was performed using the SURVEYLOGISTIC procedure in SAS to examine the effect of weight status on each risk behavior, controlling for grade, sex, and race/ethnicity. The logistic regression models were tested for goodness-of-fit by using the Hosmer-Lemeshow test using the LACKFIT option after the model statement in SAS. If the test was not significant $(\mathrm{p}$-value $>0.05)$ then the model was determined to fit the data well. In addition, R-square measure was requested by performing the RSQUARE option after the model statement. Furthermore, interaction terms (e.g., overweight*race overweight* grade overweight*sex) were entered into the model to evaluate any interactions between the main independent variable and demographic variables. If the interaction was not significant then the interaction term was not included in the final model. All analyses were performed on weighted data to adjust for nonresponse and oversampling of black and Hispanic students. Statistical tests

were considered significant at $\mathrm{p}$-value $<0.05$ and odds ratios $(\mathrm{OR})$ were considered significant if their $95 \%$ confidence intervals did not include 1.0 . 


\subsection{Results}

The total sample for this study was 13,583 high school students. Tables 1 and 2 show summary statistics of our sample's demographic and health risk behavior characteristics. In this study, more than half of adolescents were white (55\%), half of the sample was female, and the majority were between the ages of 15 and 18 years. Almost $14 \%$ were obese, and 17\% were overweight. In our overall sample, $16 \%$ of adolescents were current smokers (smoked cigarettes on 1 or more days in the past 30 days), almost a quarter (23\%) currently used marijuana (used marijuana 1 or more times during the past 30 days), and $35 \%$ had drank alcohol on one or more days in the past 30 days. During the last 30 days, $22 \%$ of adolescents rode with a driver who had been drinking alcohol, and almost a quarter had texted while driving. Almost $60 \%$ of adolescent rarely or never wore a bicycle helmet while riding a bicycle (during the past year). Thirty-four percent of adolescents were currently sexually active (had sexual intercourse with 1 or more people in the past 30 days), and of those sexually active only $18 \%$ had used a condom during their last sexual intercourse. All analyses were adjusted for the covariates.

Association of Weight Status with Cigarette, Marijuana, and Other Drug use. The relationship between cigarette use and obesity remained significant after adjusting for demographic variables $(\mathrm{AOR}=1.44,95 \% \mathrm{CI}, 1.15-1.79)$. No significant relationships were found between overweight and marijuana and other drug use (Table 3).

Association of Weight Status with Unintentional Injuries and Behaviors Contributing to Violence. Obese students were significantly less likely to engage in behaviors that lead to unintentional injury than normal weight students ( $\mathrm{AOR}=0.83 ; 95 \% \mathrm{CI}, 0.73-0.95)$. No significant differences were observed between overweight and normal weight students in regards to behaviors contributing to violence, however obese students were more likely to engage in 
behaviors contributing to violence (AOR=1.39,95\% CI, 1.24-1.57). Specifically, obese students were significantly more likely to carry a weapon $(\mathrm{AOR}=1.43,95 \% \mathrm{CI}, 1.20-1.70)$, and fight (one or more times in the past 12 months) (AOR=1.33, 95\% CI, 1.03-1.77). Additionally, obese students were more likely to be bullied at school and electronically bullied in the past 12 months $(\mathrm{AOR}=1.47,95 \% \mathrm{CI}, 1.28-1.68$ and $\mathrm{AOR}=1.20,95 \% \mathrm{CI}, 1.00-1.45)$ compared to their normal weight peers.

Association of Weight Status and Depressive Symptoms and Suicidality. Obese students, compared to normal weight students were significantly more likely to be depressed ( $\mathrm{AOR}=1.19$, 95\% CI, 1.04-1.37), have suicidal thoughts (AOR=1.51, 95\% CI, 1.31-1.76), and to have planned a suicide ( $\mathrm{AOR}=1.61,95 \% \mathrm{CI}, 1.35-1.92)$ in the past 12 months.

Association of Weight Status and Risky Sexual Behavior. After adjusting for demographics, among students those who were obese were significantly less likely to engage in risky sexual behavior (AOR=0.86, 95\% CI, 0.75-0.98) compared to normal weight adolescents. We repeated the analyses separately for each indicator of risky sexual behavior (i.e., 4+ partners in the past 12 months, no condom use, and alcohol use before last sexual intercourse) and found no significant associations by weight status.

Association of Weight Status and Unhealthy Weight Control. Among students, compared to normal weight students, overweight students were significantly more likely to engage in unhealthy weight control ( $\mathrm{AOR}=3.26,95 \% \mathrm{CI}, 2.77-3.84$ ), and obese students were even more likely to engage in unhealthy weight control compared to normal weight students ( $\mathrm{AOR}=7.56$, 95\% CI, 6.33-9.04).

Association of Weight Status and Poor Dietary Habits and Sedentary Behavior. Among students, those who were obese had significantly higher odds of having poor dietary habits ( $\mathrm{AOR}=1.37$, 
95\% CI, 1.20-1.55) and engage in sedentary behavior ( $\mathrm{AOR}=1.74,95 \% \mathrm{CI}, 1.52-1.99)$ compared to normal weight adolescents.

\subsection{Discussion}

The purpose of this study was to examine the association between weight status and health risk behaviors among high school adoelscents. We found significant associations between almost all risk behaviors we examined as outcome variables and obesity. Our results show significant relationships between obesity and smoking cigarettes (Cawley et al., 2004; Farhat et al., 2010, Ratcliff et al., 2011). Cigarette use in this population is reason for concern because it adds a second chronic disease risk factor to obesity (Lowery et al., 2014; Farhat et al., 2012). In fact, research shows that the risk effects of obesity may be intensified by cigarette use for diseases such as asthma and COPD (Buether \& Sutherland, 2007; Gilliland et al., 2003; Chen et al., 2002; Figueroa-Munoz et al., 2001).

Our findings support previous studies in that there is a significant relationship between obesity and engaging in physical fights (Pasch et al., 2008; Zeller et al., 2008), carrying of weapons (Farhat et al., 2010), bullying (Berg et al., 2005; Farhat et al., 2010; Griffiths et al. 2006; Janssen et al., 2004), unhealthy weight control (Fonseca et al., 2009; Neumark-Sztainer et al., 2002), and increased risk of depression and suicidal behavior (Eaton et al., 2005; Falkner et al., 2001; Swahn et al., 2009). We found that obese students were more likely to get bullied on school property and be cyber bullied compared to their normal weight peers. Psychosocial consequences of overweight and obesity include feelings of loneliness, sadness (Strauss, 2000), lower perceived self-worth (Vila et al., 2004), and lack of emotional well-being (Wardle \& Cooke, 2005). Our findings of the association of depression, and suicidality and obesity, also support prior findings that obese adolescents are more likely to be depressed, and have suicidal 
thoughts compared to their healthy weight peers (Dave and Rashad, 2009). Research shows that the rates of discrimination related to weight status in the U.S. are close to or higher than are the reported rates of racial discrimination, which may explain the higher rates of bullying, depression and suicidality among obese adolescents compared to their healthy weight peers (Farhat et al., 2010).

Similar to previous research, we found a negative relationship between risky sexual behavior and obesity (Lowery et al., 2014). Prior studies have indicated that BMI may be a protective factor for some health risk behaviors such as risky sexual behavior (Lowery et al., 2014; Cawley et al., 2006) because of the decreased likelihood of sexual experience and current sexual activity (Ratcliff et al., 2011). Research also shows that overweight and obese adolescents experience social, and psychological challenges more so than do their normal weight peers (Franklin et al., 2006; Puhl et al., 2007; Ratcliff et al., 2011). For example, overweight and obesity in adolescents has been associated with lower self-esteem and lower body satisfaction, which may result in feeling less secure and comfortable in sexual interactions (Lowery et al., 2014; Strauss, 2000; Puhl et al., 2007). This may result in fewer sexual experiences compared to adolescent with postivie body image (Seal et al., 2009). However, obese girls that are sexually active may be more likely to engage in risky sexual behavior such as not using condoms or other contraceptives (Averett et al., 2010; Villers, 2010) and less likely to negotiate safe sex practices (Michael, 2004) as this may prevent them from receiving negative feedback from males who tend to view sex with no condoms as more pleasurable (Espinosa-Hernandez \& Lefkowits, 2009). Our findings did not show a signficant association between weight status and specific elements of risky sexual behavior such as the number of lifetime sexual partners, and no condom use. In addition, while previous research show a significant relationship between alcohol and 
other drug use and obesity (Berg et al., 2005; Oesterle et al., 2004), our study did not find a signifcant relatioship. We found significant association between obesity and poor dietary habits and sedentary behavior, however no signficant relationships were found between overweight and poor dietary habits and sedentary behavior.

\section{Strengths and Limitations}

A strength of this current study was the large nationally representative sample of high school adoelscents with high response rates. This allows for generalizability and representativeness of these findings to high school students in the U.S. In addition, using the YRBSS data allowed us to consider a large number of variables related to risky behaviors and relevent to the priority areas outlined in Healthy People 2020 (US Department of Health and Human Services [DHHS], 2010) for this population. In addition, the use of this nationally representative dataset also minimized the potential of selection bias given that overweight obese adolescents are often identified for research participation while seeking treament for weight related concerns (i.e., weight loss and/or obesity related chronic illnesses).

This study also has a number of limitations. The cross-sectional study design of YRBSS limits our ability to infer causality. While YRBSS data is representative of high school adolescents in the U.S., it does not fully represent adolescents in the U.S. because data on adolescents who do not attend school are not captured. Although self-report of height and weight have been shown to be correlated with data on the basis of measured height and weight $(r=0.89$; mean difference, $2.6 \mathrm{~kg} / \mathrm{m}^{2}$ ), (Brener et al., 2003), self-report data on BMI tend to result in underestimation of weight and overestimation of height. YRBSS does not include important socio-demographic (e.g., socioeconomic status) and contextual variables (e.g., family structure) that may influence risk taking behaviors in overweight obese adolescents. 


\section{Implications and Future Research}

Although prior research suggests that obese adolescents may be more prone to social isolation compared to healthy weight adolescents (Strauss \& Pollack, 2006; Zeller et al., 2008), because they are less likely to be exposed to or engage in what some research has identified as normative adolescent risk behaviors (Zeller et al., 2008) our results however show that there is reason for concern given that obese adolescents in most cases reported engaging in high risk behaviors even more than healthy weight adolescents. Health care providers and public health professionals addressing weight-related concerns in adolescents should also assess for other health risk behaviors and develop tailored prevention and intervention programs that meet the needs of this subgroup of adolescents. Further research using longitudinal study designs are needed to ascertain any temporal sequence between overweight and obesity and risk taking behaviors among adolescents taking into account contextual (e.g., family structure, parental education, peer-support) and sociodemographic factors (e.g., socioeconomic status, grade, and race/ethnicity). Recognizing that obesity is a multidimensional public health problem that involves a complex configuration of behavioral, biological, and socioenvironmental factors, interventions and health promotion efforts targeted at overweight and obese adolescents need to consider other factors in addition to the recognition of the amount of control adolescents have over their time, nutrition and diet and address risk-taking behavior among this group of adolescents. There is growing acknowledgement that programs that address multiple risk behaviors may show the most potential for preventing and addressing obesity in adolescents (Peterson \& Fox, 2007; Windle et al., 2004). Interventions that seek to promote healthy diet within the context of weight management may have more of an impact if they integrate healthy diet/nutrition and physical activity strategies along with strategies to address additional risk 
behaviors such as depressive symptoms or tobacco use as components of the prevention or intervention programs. 


\title{
Chapter 5
}

\section{Factors associated with tobacco use, poor dietary habits, and physical inactivity and sedentary behavior among high school adolescents in the U.S.}

\begin{abstract}
Objective: The purpose of this study was to examine the association between tobacco use, poor dietary habits, physical inactivity and sedentary behavior among high school adolescents in the United States, taking into account weight status and sociodemographic factors.

Methods: Secondary data analysis was conducted using the 2013 Youth Risk Behavior Surveillance Systems (YRBSS) dataset. The study population consisted of 13,583 high school adolescents. Odds ratios and adjusted odds ratios were calculated using logistic regression. All analyses were conducted using the Proc Survey procedure in SAS 9.4.

Results: The prevalence of tobacco use among adolescents was (22\%), seven percent of adolescent ate no vegetables, per day in the past seven days and five percent did not eat any fruit including $100 \%$ fruit juice in the past seven days prior to taking the survey. Results from the logistic regression analysis showed that after controlling for weight status and sociodemographic factors, for both male and female adolescents there was a significant association between particular indicators of poor dietary behavior and current tobacco use. Girls who skipped breakfast every day in the past seven days and drank soda two times or more a day for the past seven days were significantly more likely to also use tobacco compared to their counterparts $(\mathrm{AOR}=2.14,95 \% \mathrm{CI} 1.68-2.73)$. Similar to the girls, among boys there was a significant relationship between skipping breakfast, and drinking soda and tobacco use. Boys who skipped breakfast every day in the past week were significantly more likely to also use tobacco compared to boys who did not skip breakfast (AOR $=1.54,95 \%$ CI 1.16-2.03). Among boys, those who drank soda twice or more per day during the past seven days were twice as likely to use tobacco $(\mathrm{AOR}=2.02,95 \% \mathrm{CI} 1.74-2.35)$. There was a significant inverse relationship between playing excessive video or computer games (i.e., $>3$ hours per day) and tobacco use among boys (Table 3). Results also showed that there was a significant inverse relationship between tobacco use and physical inactivity among boys. Boys who did not engage in one hour of physical activity per day in the past seven days were significantly less likely to use tobacco compared to boys who did engage in physical activity in the past seven days (AOR $=0.62,95 \%$ CI 0.49-0.79).

Conclusion: This study found an association between poor dietary habits, sedentary behavior, and tobacco use among adolescents. The findings highlight the importance of and the need for interventions that promote healthy dietary behavior and physical activity along with tobacco cessation efforts.
\end{abstract}

Keywords: adolescents, tobacco use, physical inactivity, poor dietary habits, sedentary behavior, YRBSS 


\subsection{Introduction}

Tobacco use, poor dietary behavior, and physical inactivity have been identified as major contributors of morbidity and mortality in the United States (U.S. Department of Health and Human Services [DHHS], 2010). These modifiable health risk behaviors, prevalent in adolescents, have been associated with adverse health outcomes such as coronary heart disease, and metabolic diseases such as diabetes mellitus, pulmonary diseases, impaired immune function, rheumatoid arthritis, certain cancers, and other chronic illnesses (DHHS, 2014; Larson et al., 2007; Wilson et al., 2005).

In 2013, almost a quarter of high school adolescents were current tobacco users (including cigarette use, current smokeless tobacco use, or current cigar use) during the 30 days before the survey (Kann et al., 2014). National survey data also indicate that the majority of adolescents in the U.S. do not have diets that meet the recommended dietary guidelines in the U.S. (DHHS, 2015). In addition to tobacco use, and poor dietary habits, sedentary behavior has also emerged as a public health issue among adolescents (Pearson et al., 2011; Owen et al. 2010; DHHS, 2014). Some researchers define "sedentary" as not meeting a criterion level of physical activity (e.g. the Healthy People 2020 guidelines recommend at least 60 minutes each day of aerobic physical activity for children and adolescents). However, sedentary behavior also includes excessive sitting time or very low levels of energy expenditure through sitting and laying down (Owen et al., 2010). The Healthy People 2020 physical activity objectives address sedentary behaviors by calling for an increase in the proportion of children and adolescents who do not exceed recommended limits for screen time, including viewing television less than three hours a day and using a computer or playing computer games outside of school (for non-school work) less than three hours a day (DHHS, 2010). 
Despite evidence showing that regular physical activity improves health and quality of life, approximately one out of three adolescents are either overweight or obese (Kann et al., 2014). Researchers have indicated concern that sedentary behavior as a result of excessive television viewing and playing video games among children and adolescents could lead to adverse health effects such as obesity, poor physical fitness, and substance use (Hancox et al., 2004; Strasburger, Jordan, \& Donnerstein, 2010; Johnson et al., 2002).

Although the relationship between tobacco use, poor diet, and lack of exercise have been investigated extensively among adults (Anthony et al., 2011; Chow et al., 2010; Lando et al., 1999; Yusuf et al., 2004; Katzmarzyk et al., 2009), previous research in adolescents has mostly focused on examining the association of tobacco use with individual behavioral factors such as physical activity (Dvorak et al., 2009; Moore et al., 2005), poor nutrition (Korhonen et al., 2009; Ma et al., 2000), and other health risk behaviors such as risky sexual behavior (Lowery et al., 2014), and alcohol and other drug use (Respress et al., 2013; Wallace et al., 2011).

Few studies have examined the relationship and overlap between tobacco use, poor dietary habits, and physical inactivity, and sedentary behavior among adolescents (Larson et al., 2007; Wilson et al., 2005). Larson and colleagues (2007) used data from Project EAT (Eating Among Teens) to describe smoking frequency and its relationship to physical activity and dietary patterns among middle school and high school students from Minneapolis-St. Paul public schools. The study reported that smoking in adolescents was inversely associated with physical activity and healthy diet. Another study examined the associations between adolescent smoking and consumption of fruit, vegetable, and dairy products, and frequency of exercise in a sample of adolescents in Virginia (Wilson et al., 2005). Both of these studies used state-level data. To our knowledge, no study has examined the association between tobacco use (including cigars, 
chewing tobacco, snuff, or dip), poor dietary habits, and physical inactivity, and sedentary behavior using nationally representative data. In this study, we aim to address this critical gap by using a national dataset to test the association of tobacco use, indicators of poor dietary habits such as no consumption of fruit, vegetable, milk, breakfast and consumption of soda, and physical inactivity and sedentary behavior, taking into account race/ethnicity, grade, weight status, and depressive symptomology. Recognizing that there is a difference in the prevalence and association of each of predictors and our outcome variable individually by gender, we stratified our analyses to present findings for male and female students separately.

\subsection{Methods}

A secondary data analysis of the 2013 Youth Risk Behavior Surveillance System (YRBSS) was conducted to address the aim of this study. The YRBSS national survey given to adolescents in grades 9-12 and is conducted by the CDC biennially. YRBSS focuses on six main areas of interest: 1) behaviors that contribute to unintentional injuries and violence; 2) sexual behaviors that contribute to sexually transmitted infections including HIV, and unintended pregnancy; 3) tobacco use; 4) alcohol and other drug use; 5) unhealthy dietary behaviors; and 6) physical inactivity (CDC, 2015). YRBSS uses a three-stage, cluster sample design to obtain a nationally representative sample of U.S. adolescents. The target population of the YRBSS includes all public and private school students in grades 9-12 in the 50 states and the District of Columbia. YRBSS applies a weight variable created based on student sex, race/ethnicity, and school grade to each record to adjust for student nonresponse and oversampling of black and Hispanic students. This procedure makes the weighted estimates representative of all adolescents attending schools in the U.S. Additional information about the sudy design and data collection of YRBSS is discussed elsewhere (Brener et al., 2013). 
The variables included in the current study were tobacco use (including cigarettes, cigars, chewing tobacco, snuff, or dip on one or more of the past 30 days), indictors of poor dietary behavior (no vegetable, fruit, milk intake, drinking soda 2+ times per day, skipping breakfast), and indicators of physical inactivity (students who were physically active for a total of 60 minutes per day on zero of the past seven days, students who watched TV, and played video or computer games for more than 3 hours per day on an average school day).

\section{Covariates}

Covariates in this study included depression and weight status based on Body Mass Index (BMI). Demographic variables included age, sex, grade, and race/ethnicity.

Chronological age was assessed using the participants' self-reported age at the time the YRBS was conducted. Gender was a self-reported measure of either male or female and was measured as a dichotomous variable based on the YRBSS question "What is your sex?" Grade level was also a self-reported measure of the current grade level at the time the survey was completed and it was measured as an ordinal variable $\left(9^{\text {th }}\right.$ grade, $10^{\text {th }}$ grade, $11^{\text {th }}$ grade, and $12^{\text {th }}$ grade) based on the YRBSS question "In what grade are you?" Race/ethnicity was measured as a self-reported nominal variable based on two questions in the YRBSS, "Are you Hispanic? Yes/No" and "What is your race? American Indian or Alaska Native; Asian; Black or African American; Native Hawaiian or Other Pacific Islander; and White.” We grouped race/ethnicity into five cateogries of white, black, Hispanic, multiple-Hispanic, and other (American Indian, Pacific Islander/Hawaiian Native, Asian, and multiple non-Hispanic). 
Depression was measured as a dichotomous variable based on the question "During the past 12 months, did you ever feel so sad or hopeless almost every day for two weeks or more in a row that you stopped doing some usual activities?' (Answer options 'Yes' or 'No')Weight status was measured as a YRBSS constructed dichotomous variable as overweight $\left(\geq 85^{\text {th }}\right.$ to $<95^{\text {th }}$ percentile of BMI) and obese $\left(\geq 95^{\text {th }}\right.$ percentile of BMI).

Tobacco Use was assessed based on the YRBSS constructed dichotomous variable that indicate the "percentage of students who smoked cigarettes, or cigars, or used chewing tobacco, snuff, or dip on one or more of the past 30 days. Yes/No"

Poor dietary habits were assessed based on YRBSS constructed dichotomous variables that indicate "the percentage of students who ate or drank $100 \%$ fruit juices 0 times per day during the past 7 days," "the percentage of students who ate vegetables 0 times per day during the past 7 days," “... who drank a can, bottle, or glass of soda or pop two or more times per day during the past 7 days," ".... who drank 0 glasses per day of milk during the past 7 days," and " the percentage of students who ate breakfast on none of the past 7 days."

Physical inactivity, and sedentary behavior were assessed based on YRBSS constructed dichotomous variables that indicate "the percentage of students who were physically active for a total of 60 minutes on 0 of the past 7 days," "the percentage of students who watched three or more hours per day of TV on an average school day," and "percentage of students who played video games or used a computer for something that was not school work three or more hours per day on an average school day."

We used YRBSS constructed dichotomous variables for consumption of fruits and vegetables, physical inactivity and sedentary behavior with cut points chosen consistent with previous literature and the 2020 health objectives (Healthy People 2020). 


\section{Statistical Analysis}

All analyses were conducted in SAS version 9.4 (SAS Institute, Inc, Cary, NC) using PROC SURVEY procedures to take into account the complex survey design in order to calculate weighted percentages and to correct for oversampling. Descriptive statistics including frequencies and percentages, using the SURVEYFREQ procedure in SAS, were used to determine the sample demographic and behavioral characteristics. Chi-square tests were performed to assess for bivariate associations of tobacco use with all the independent variables for male and female adolescents. Multivariable logistic regression was performed, using the SURVEYLOGISTIC procedure in SAS, to obtain adjusted odds ratios (AOR) for the association of tobacco use with poor dietary habits and physical inactivity and sedentary behavior separately for males and females and adjusted for all statistically significant covariates from the bivariate analyses. The bivariate analysis showed a significant relationship between tobacco use and depression for both males and females, therefore, depression was adjusted for in the final model. We also included an interaction term for physical inactivity and playing videogames in the final model. Logistic regression assumptions were tested by reviewing the number of observations, model fit statistics (i.e. AIC), models' goodness-of-fit by adding the RSQUARE option after the model statement in SAS. All statistical tests were considered significant at p-value $<0.05$ and odds ratios (OR) were considered significant if their 95\% confidence Intervals did not include 1.0. 


\subsection{Results}

The current study sample consisted of 13,583 high school adolescents. As shown in Table 1, half of the population was male, and over half of the population was white (56\%). Each grade was equally represented, with slightly over a quarter (27\%) comprised of students in the $9^{\text {th }}$ grade. In addition, $17 \%$ of the population reported being overweight and 14\% reported being obese.

Nearly a quarter $(22 \%)$ of adolescents reported tobacco use one or more times in the last 30 days. Approximately, $7 \%$ of adolescents reported not consuming any vegetables in the past seven days, $14 \%$ did not eat breakfast on any day in the 7 days prior to the survey, and $19 \%$ drank a can, bottle, or glass of soda or pop two or more times per day during the past 7 days. Fifteen percent of adolescents did not participate in any physical activity for a total of 60 minutes per day in the past seven days, a third (32\%) watched three or more hours of television per day on an average school day, while $41 \%$ reported playing video or computer games for more than three hours per day on an average school day (Table 1).

\section{Regression Analyses}

Among female students there was a significant relationship between tobacco use and indicators of poor dietary habits. There was an inverse relationship between no vegetable intake and tobacco use among girls $(\mathrm{AOR}=0.56,95 \% \mathrm{CI} 0.37-0.84)$. Girls who skipped breakfast every day in the past seven days were significantly more likely to use tobacco (AOR $=1.32,95 \%$ CI 1.06-1.64), and those who drank a can, bottle, or glass of soda two or more times per day during in the past seven days were twice as likely to use tobacco (AOR $=2.14,95 \%$ CI 1.68 2.73) compared to students who did not drink soda two more times a day. The results showed no significant relationship between physical inactivity/sedentary behavior and tobacco use among girls. 
Similar to the girls, among boys there was a significant relationship between skipping breakfast, and drinking soda and tobacco use (Table 3). Boys who skipped breakfast every day in the past week were significantly more likely to also use tobacco compared to boys who did not skip breakfast $(\mathrm{AOR}=1.54,95 \%$ CI 1.16-2.03). Among boys, those who drank soda twice or more per day during the past seven days were twice as likely to use tobacco ( $\mathrm{AOR}=2.02,95 \%$ CI 1.74-2.35).

There was a significant inverse relationship between playing excessive video or computer games (i.e., $>3$ hours per day) and tobacco use among boys (Table 3). Results also showed that there was a significant inverse relationship between tobacco use and physical inactivity among boys. Boys who did not engage in any physical activity in one hour of physical activity per day in the past seven days were significantly less likely to use tobacco compared to boys who did engage in physical activity in the past seven days $(\mathrm{AOR}=0.62,95 \% \mathrm{CI} 0.49-0.79)$.

\subsection{Discussion}

The current study examined the relationship between tobacco use, poor dietary habits, and physical inactivity and sedentary behavior among high school adolescents in the U.S. The principal findings of this study are that among both male and female adolescents there is a significant association between particular indicators of poor dietary behavior and current tobacco use. Among adolescents, girls who skipped breakfast every day in the past seven days and drank soda two times or more a day for the past seven days were significantly more likely to also use tobacco compared to their counterparts. These findings are consistent with previous literature (Wilson et al., 2005; Sallis et al., 2000). Similar to our findings, Wilson et al found that female smokers were significantly less likely to report eating one or more servings of vegetables per day than nonsmokers. Interestingly, in our study the unadjusted model for girls showed that 
adolescents who consumed vegetables were almost twice as likely to use tobacco, however this changed to an inverse relationship once we adjusted for our other covariates (race/ethnicity, grade, BMI, and depression) $(\mathrm{OR}=1.82,95 \%$ CI 1.31-2.55; AOR 0.56, 95\% CI 0.37-0.84, respectively).

Similar to previous research, we found a positive association between skipping breakfast and tobacco use among both boys and girls (Keski-Rahkonen et al., 2003). Skipping breakfast in adolescents has been associated with various health risk behaviors including tobacco, alcohol, and substance use (Isralowitz \& Trostler, 1996; Ho“"glund et al., 1998). Studies have also shown that adolescent girls skip breakfast more often than boys (Isralowitz \&Trostler, 1996; KeskiRahkonen et al., 2003) suggesting that skipping breakfast for girls may be a method of weight control (Keski-Rahkonen et al., 2003). Our study found no gender difference in the prevalence of skipping breakfast.

Contrary to other research (Hancox et al., 2004; Strasburger et al., 2010), we did not find a significant association between television viewing and tobacco use among adolescents in our sample. We found a significant inverse relationship between tobacco use and playing video or computer games among boys but not girls. This may relate to the availability and use of a variety of electronic devices and new media tools such as iPhone, iPad, and laptops among adolescents (Rideout, Foehr, \& Roberts, 2010). Approximately $41 \%$ of adolescents in our sample played video or computer games and 32\% reported watching TV for three or more hours a day on an average school day. Numerous studies have linked TV viewing with increased risk for overweight and obesity among children and teens (Tremblay et al., 2011; Ekelund et al., 2006; Pearson et al., 2011; Salmon et al., 2011). There is also evidence about the adverse effects of watching more TV on children's health as they grow older. Troiano et al (2008) note that 
children and adolescents who spend more than two hours per day watching TV are more likely to be overweight, have higher cholesterol levels, smoke, and have poor fitness as adults. There is a need for using objective measures to assess not only watching TV and playing video games but also sitting time during school, or at home as sedentary behavior among this population.

\section{Strengths and Limitation}

A strength of this current study is the large nationally representative sample of high school adoelscents with high response rates that allows for generalizability and representativeness of these findings to high school students in the U.S.

There are several limitations to our study. First, this study is based on a cross sectional study design. Second, although the study uses a complex survey design and can be generalized to high school students nationally, these data only apply to adolescents who attend school and therefore are not representative of all adolescents in the U.S. Third, although the YRBSS questionnaire demonstrates good test-retest reliability (Brener et al., 2003), the extent of over or underreporting of self-reported behaviors such as physical activity, poor dietary habits, weight and height, and tobacco use could not be determined. Fifth, important variables such as socioeconomic status, and parental smoking and eating behavior not accounted for in the YRBSS were not considered in our study.

\section{Implications and Future Research}

Despite these limitations, our results based on a nationally representative sample of adolescents show that there is an association between poor dietary habits, physical inactivity and sedentary behavior, and tobacco use in this population. These findings may have important policy and practice implications. There is evidence that adolescents who engage in one health risk behavior may engage in other health risk behaviors, therefore, comprehensive interventions 
that address multiple risk behaviors among this population maybe more effective. One such intervention is the Not On Tobacco (N.O.T) smoking cessation program, designed for adolescents who are daily smokers. In addition to smoking cessation, this program also promotes healthy lifestyle behaviors such as physical activity, and healthy eating, stress management, decision making, and social support skills (Horn et al., 2013).

Despite the inverse relationship of sedentary behavior and tobacco use in our study, the high prevalence of sedentary behavior among adolescents is alarming and calls for more research and interventions that encourage adolescents to not only increase their level of physical activity but to also reduce the amount of time they engage in sedentary behavior such as watching TV and playing video games. Further research using multiple sources of data collecting information on parental eating and smoking behavior, and using psychometric approaches to collect information are needed to provide validated findings. Furthermore, efforts to reverse and prevent the nation's child and adolescent obesity epidemic should include more focus on reducing youth's sedentary behavior, especially time spent watching TV, playing video or computer games, and sitting time in school and at home. 


\section{Chapter 6 \\ Discussion}

\subsection{Summary of key findings}

The current dissertation project utilized nationally representative data from the Youth Risk Behavior Surveillance System (YRBSS) conducted between 2012 and 2013 in high schools in the United States. Problem Behavior Theory (PBT) was used as the theoretical framework to assess the pattern and co-occurrence of risky behaviors among high school adolescents in the U.S. Specifically, the project aimed to examine 1) the co-occurrence of health risk behaviors among adolescents and the pattern of risk behaviors by sex, 2) the association of health risk behaviors and weight status (overweight and obesity), and 3) the association of tobacco use, poor dietary habits, physical inactivity, and sedentary behavior among adolescents controlling for weight status, and sociodemographic variables.

In the first study (aim 1), our analyses yielded five subgroups of youth engaging in risky behavior: those not engaging in any risky behavior - low risk (Classes 1 and 2), those with depressive symptoms and suicidal thoughts (Class 3), those with high probability of tobacco and alcohol use (Class 4), and those identified as polysubstance users - highest-risk subgroup(Class 5). Similar to previous research we found that the majority of high school adolescents belonged to the low risk subgroup (Zweig et al., 2001; Pena et al., 2012; Connell et al., 2009) and this was true for both boys and girls (62\% for boys and 50\% for girls). The highest risk profiles for both boys and girls accounted for the lowest percentage of adolescents (12.2\% for boys and $11.6 \%$ for girls). Supporting previous research which linked two or three risk behaviors (Zweig et al., 2001; Durkham et al., 1996; Shrier et al., 1996; Burge et al., 1995) we found that adolescents in the highest-risk subgroups engaged in multiple risk behaviors including tobacco, alcohol, marijuana, 
prescription drug use, risky sexual behavior, and behaviors that lead to unintentional injuries and violence. For both boys and girls, a polysubstance use-high-risk subgroup was identified providing not only more evidence of the interrelationship between substance use and risky sexual behavior, but also the links between different types of substances and engaging in behaviors such as texting and driving, and riding with a drunk driver, and getting into physical fights. Despite the similarities in subgroups of risky behavior for boys and girls, there were differences in the levels of risk and the types of behaviors adolescents engaged in. Only girls reported high rates of depressive symptoms, suicidal thoughts, and plans to attempt suicide, and moderate rate of engaging in sedentary behavior (playing video games three or more hours a day). While girls in the polysubstance use high risk subgroup, reported high rates of depressive symptoms and suicidality along with the majority of other risk behaviors, boys in the polysubstance use subgroup did not report depressive symptoms and suicidal thoughts and attempts. These findings highlight that depression and suicidal ideation are prevalent among adolescents and often cooccur with a multitude of other risky behaviors (i.e., tobacco, alcohol, marijuana, and prescription drug use, risky sexual activity). Our results also indicate differences in subgroups of adolescents that suggest important interrelationships and variations in risk behaviors across the different subgroups as well as by sex. Future research is needed to examine health risk behaviors using well-designed nationally representative longitudinal studies that include contextual variables in the analyses to possibly identify risk and protective factors leading to behavioral choices among adolescents. Knowledge of subgroup differences in the prevalence of the cooccurrence of risk behaviors can help public health practitioners and policymakers to develop targeted health campaigns, and interventions to at-risk subgroups of adolescents. 
The second study (aim 2) found significant associations between almost all risk behaviors examined as outcome variables in this study and obesity. Among adolescents, those who were obese were more likely to smoke cigarettes, engage in behaviors leading to violence, more likely to be depressed, suicidal, bullied, engage in unhealthy weight control, and sedentary behavior. We also found a negative relationship between risky sexual behavior and obesity. Similar to previous research (Farhat et al., 2010; Zeller et al., 2008), we found that obese students were more likely to get bullied on school property and cyber bullied compared to their normal weight peers. Our findings of the association of depression, and suicidality also support prior findings that obese adolescents are more likely to be depressed, and have suicidal thoughts compared to their healthy weight peers. Unlike previous research, we did not find a signficant relationship between alcohol and drug use and overweight obesity (Oesterle et al., 2004). We found significant association between obesity and poor dietary habits and sedentary behavior, however no signficant relationships were found between overweight and poor dietary habits and sedentary behavior. Comprehensive and longitudinal studies are needed to ascertain any temporal sequence between obesity and risk taking behaviors among adolescents.

In the third study (aim 3), we found that among both male and female adolescents there is a significant association between particular indicators of poor dietary behavior and current tobacco use. Among adolescents, girls who skipped breakfast every day in the past seven days and drank soda two times or more a day for the past seven days were significantly more likely to also use tobacco compared to their counterparts. These findings are consistent with previous literature (Wilson et al., 2005; Sallis et al., 2000). Similar to our findings, Wilson et al found that female smokers were significantly less likely to report eating one or more servings of vegetables per day than nonsmokers. Interestingly, in our study our model adjusted for race/ethnicity, grade, 
BMI, and depression for girls showed an inverse relationship between vegetable consumption and tobacco use (AOR 0.56, 95\% CI 0.37-0.84, respectively). The relationship was not found among boys.

Similar to previous research we found a positive association between skipping breakfast and tobacco use among both boys and girls (Keski-Rahkonen et al., 2003). Skipping breakfast in adolescents has been associated with various health risk behaviors including tobacco, alcohol, and substance use (Isralowitz \& Trostler, 1996; Ho“glund et al., 1998). Studies have also shown that adolescent girls skip breakfast more often than boys (Isralowitz \&Trostler, 1996; KeskiRahkonen et al., 2003) suggesting that skipping breakfast for girls may be a method of weight control (Keski-Rahkonen et al., 2003). Our study found no gender difference in the prevalence of skipping breakfast. We found a significant inverse relationship between tobacco use and playing video or computer games among boys but not girls. This may relate to the availability and use of a variety of electronic devices and new media tools such as iPhone, iPad, and laptops among adolescents (Rideout, Foehr, \& Roberts, 2010).

Our research findings based on all three studies (chapters 3-5) contribute to our understanding of adolescent risk behaviors and identifies patterns of risky behaviors among adolescents supporting the PBT by demonstrating that health risk behaviors do co-occur. All three studies show that chronic disease risk behavior such as tobacco use, sedentary behavior, and obesity as a risk factor, are prevalent in this population. Many chronic diseases of adulthood, including certain cancers, cardiovascular disease (CVD), and type 2 diabetes are associated with health risk behaviors such as tobacco use, sedentary behavior, physical inactivity, and poor dietary behavior (Ogden et al., 2014). The first study identified distinct patterns of the cooccurrence of risk behaviors among high school boys and girls. The findings, supporting the PBT 
premise of co-occurring risky behaviors, showed that adolescents in the highest-risk subgroups engaged in multiple risk behaviors including tobacco, alcohol, marijuana, prescription drug use, risky sexual behavior, and behaviors that lead to unintentional injuries and violence.

The second study examined the association of the same set of risk behaviors stratified by weight status. Findings showed that obese students were more likely to smoke cigarettes, engage in behaviors leading to violence, more likely to be depressed, suicidal, bullied, engage in unhealthy weight control and sedentary behavior. The third study examined the relationship between tobacco use, poor dietary habits, and physical inactivity, as risk behaviors associated with adolescent and adult chronic diseases. The findings showed positive associations between poor dietary behavior and tobacco use. High prevalence of tobacco use and cigarette use was found in all three studies.

Depression and suicidality emerged as a common themes in studies one and two. The first study showed that only girls reported high rates of depressive symptoms, suicidal thoughts, and plans to attempt suicide, and moderate rate of engaging in sedentary behavior (playing video games three or more hours a day). Further, while girls in the polysubstance use high risk subgroup, reported high rates of depressive symptoms and suicidality along with the majority of other risk behaviors, boys in the polysubstance use subgroup relatively lower rates of depressive symptoms and suicidal thoughts and attempts. The second study showed significant association between depression and suicidality and obesity.

As evident by our research findings, by recognizing adolescent risk behavior or problem behavior as part of the behavior system which stems from the interaction between personality and environment systems - we can strive to develop more effective prevention and intervention programs. PBT shows that problem behaviors are related and that any single problem behavior, 
such as alcohol use, illicit drug abuse, aggressive behavior, and others must be viewed within the complex system of problem behavior, personality, and perceived environment. Prevention and intervention programs and policies are most often developed to address one area such as pregnancy prevention, tobacco cessation, or violence prevention. However, as our research shows adolescents often engage in multiple risky behaviors. Using a more comprehensive strategy in the design of program or policies may show more positive effects than addressing risk behaviors in silos. For example, Teen Outreach Program (TOP) is an evidence-based program that empowers at risk teens with the tools and opportunities to avoid risky behaviors such as drug use, violence, teen pregnancy (Child Trends, 2016). A more integrated approach to prevention and intervention efforts targeting multiple forms of risk behaviors are needed and multiple contexts of adolescents' lives including family, peer, school, community, and social media interactions need to be considered.

Our findings suggest that future research should focus on sex differences in combination with race/ethnicity, socioeconomic status, and rurality in addressing risk taking behaviors and the pattern of those risk behaviors among adolescents. Our findings show that for adolescent girls, the co-occurrence of depression and suicidality with polysubstance use are major issues. Adolescent suicide is a preventable public health problem that has increased over the past few decades among adolescents and young adults. Our findings suggest that prevention and intervention efforts screen to identify high-risk subgroups of adolescents engaging in substance use behavior and showing depressive symptoms. Studies suggest a positive association between smoking cigarettes and depressive symptoms (Nezami et al., 2005), and alcohol use and depressive symptoms (Aseltine et al., 2009; Strachman et al., 2009). 
Today, virtually every behavioral health concerns relate in some way to media influence during childhood and adolescence. Adolescents spend more time with media than any other activity - it should not be surprising that messages and images in the media influence their knowledge, attitudes, behavior about violence, sex, eating habits, obesity, and substance use (Strasburger et al., 2009). Strasburger and colleagues (2009) label media as a super peer, giving teens a glimpse into the world of sex, drugs, and relationships. Adolescent health researchers and health care providers must recognize and address key issues that may be influenced by media use including social networking sites, video games, etc. on physical activity, bullying, sexual activity, alcohol use, tobacco use, drug use, body image and eating disorder, violence, family, and social interaction.

The relationship between cigarette smoking and depression has been explained in several ways. The idea of self-medication is one hypothesis stating that individuals choose to selfmedicate using certain substances as a method to alleviate their anxiety and distress (Minnix et al., 2011). Others suggest that depression increases adolescents' likelihood of becoming smokers by increasing susceptibility to peer pressure from those who are already smokers (Patton et al., 1998; Morrell, Cohen, \& McChargue, 2010). One study using longitudinal data found that even after controlling for prior level of depression, adolescents who were smokers were more likely to experience depressive symptoms (Steuber \& Danner, 2006). More research is needed to explain the mechanisms responsible for the relationship between cigarette smoking and depressive symptoms, however, we know through extensive research that a relationship exists between smoking and depression and our study shows these behaviors tend to co-occur in this population. The association between alcohol use and depression is well documented (Aseltine et al., 2009; Botticello, 2006; Kessler et al., 1996; Regier et al., 1990; Helzer \& Pryzbeck, 1988). In addition, 
epidemiological studies show substance use including alcohol use as a risk factor for suicide attempts and completion among both adolescents and adults (Aseltine et al., 2009; Strachman et al., 2009).

Considering that substance use and mental health issues such as depression and suicidality are often related to psychosocial and environmental factors such as family situations, social support, and self-esteem, prevention, intervention, and health promotion efforts should consider these contextual factors in the design and implmentation of these efforts. For example, preventive programs may consider the stress and anxiety that often result from substance use, such as problems in school, conflict with family members, legal problems, and that all these issues may increase the likelihood of suicidal thoughts and attempts in this population.

\subsection{Strengths and limitations}

One of the main strengths of this project is utilization of a dataset that is nationally representative of the high school adolescents in the U.S. Our analyses use measures of risk that have been validated over the years (Brener et al., 2003). Complex sampling designs were able to account for in analyses for each of the three studies (aims). Another strength is the high response rate for the 2013 YRBSS, 77\% for schools, and 88\% for students. In addition, each of our three studies add to the literature and knowledge base in regards to the interrelationships of adolescent risk behaviors, the association of risk behaviors and weight status among adolescents, and the association between tobacco use, poor dietary habits, and sedentary behavior among adolescents. To our knowledge no nationally representative study has examined the variety of indicators of risk behaviors we tested in our research.

Although our project is based on a nationally representative sample of high school adolescents in the U.S. and it contributes to the knowledge about adolescent risk behaviors, it 
does have a number of limitations. YRBSS data are cross-sectional, therefore, causality cannot be established. In addition, despite previous studies suggesting that YRBSS questionnaire items have produced valid responses and have high degree of reliability (Brener et al., 2003), the survey data are self-reported and the extent of underreporting or over reporting of behaviors cannot be determined. Our results are generalizable to adolescents who attend school, therefore not representative of all adolescents. Nationwide, in 2009, approximately four percent of adolescents aged 16-17 years were not enrolled in a high school program and had not completed high school (Chapman, Laird, Ifill, \& KewalRamani, 2011). The National Health Interview Survey (NHIS) and Youth Risk Behavior Supplement conducted in 1992 demonstrated that outof-school youths are more likely than youths attending school to engage in the majority of health-risk behaviors (Brener et al., 2003). Finally, YRBSS addresses only behaviors that contribute to the leading causes of morbidity and mortality among youths and adults and not the determinants of those behaviors. Lack of information on other potential covariates such as socioeconomic status (SES), family structure, peer support, and parental education is an overall limitation for all three aims in this project. Thus, another limitation is that this project only analyzed data pertaining to demographic and behavioral characteristics of adolescents. However our research does provide evidence in respect to the interrelationships of risk behaviors among adolescents which underscores the need for prevention and intervention efforts that address multiple risk behaviors concurrently. Important psychosocial, personality related factors, and socioenvironmental factors that may impact adolescents' health risk and protective behavioral choices as suggested by the problem behavior theory, were not explored. 


\subsection{Implications}

Our study findings provide impetus for the development of prevention programs and interventions that address the co-occurrence of risk behaviors among adolescents, especially targeting high risk subgroups of adolescents. Programs designed to address multiple risk behaviors must consider demographic factors as different groups of adolescents may engage in various forms of risky behavior. Study findings also highlight that tobacco, alcohol use, and depression among youth remain important public health issues that need to be understood and addressed concurrently and appropriately taking into account sex differences in the pattern of these risk behaviors.

We suggest the following implications for public health practice and policy based on our research findings. Behavioral health practices should consider the co-occurrence of health risk behaviors in adolescents and screen for several risk behaviors simultaneously to increase the adolescent and public health professionals' (including school nurses') awareness to a situation that otherwise may not be discussed or disclosed. The knowledge gained from this study may be utilized by child and adolescent mental health professionals, to also advocate for increased contact with adolescents in pediatricians', school nurses', and psychologists' offices, to screen for behavioral and mental health issues such as substance use, risky sexual behavior, bullying, depression, and suicidal ideation.

\section{Future Research}

A significant test of PBT would be research that prospectively and longitudinally examines the interrelationships of health risk behaviors among adolescents using all theory elements to investigate the influence of psychosocial and personality related factors, and perceived environmental contexts in which adolescents make health risk and health protective 
behavioral decisions. Furthermore, research is needed to provide theoretical explanations for adolescent risk behaviors that include social determinants related to race/ethnicity and sexual minority groups. Useful theoretical approaches would be a social determinants of health model and elements of problem behavior theory. Latent class and latent profile analyses may be used to not only identify subgroups of adolescents based on the patterns of the co-occurrence of risk behaviors stratified by demographic and contextual variables, but also to identify subgroups based on the level of engagement in certain risk behaviors (e.g., identify classes of girls based on the levels of physical and psychological victimization types).

Quantitative data can be complemented by qualitative data to provide a deeper understanding of risk behaviors among adolescents. We know from previous research that societal conceptualizations of race, for example, have influenced socioeconomic status, educational status, housing status, and experiences of discrimination and stigmatization, that are often outside of an individual's control. Findings from such research may provide an additional layer to the discussion of health disparities among adolescent minority populations that deepen our understanding of variations in risk behavior prevalence among different demographic groups. Recently, National Institute of Minority Health Disparities (NIMHD) classified sexual and gender minorities as a health disparity population which means that lesbian, gay, bisexual, and transgender (LGBT) research will now be eligible for health disparity funding from any institute within the National Institutes of Health (NIH), opening opportunities to investigate health risk behaviors among LGBT adolescents.

Our charge as public health professionals is to consider individual, psychosocial, and environmental contexts of life in the $21^{\text {st }}$ century and how they effects adolescents' decision 
making regarding their health and quality of life in our prevention, intervention, and health promotion efforts while addressing risk behavioral among adolescents. 


\section{References}

\section{Chapters $1 \& 2$}

Ajzen, I. (1991). The theory of planned behavior. Organizational Behavior and Human Decision Processes, 50(2), 179-211.

Ajzen, I. (2011). The theory of planned behaviour: reactions and reflections. Psychology \& Health, 26(9), 1113-1127.

Albarracín, D., Gillette, J. C., Earl, A. N., Glasman, L. R., Durantini, M. R., \& Ho, M.-H. (2005). A test of major assumptions about behavior change: a comprehensive look at the effects of passive and active HIV-prevention interventions since the beginning of the epidemic. Psychological Bulletin, 131(6), 856.

Allen, J. P., Leadbeater, B. J., \& Aber, J. L. (1994). The development of problem behavior syndromes in at-risk adolescents. Development and Psychopathology, 6(02), 323-342.

Allison, P. D. (2012). Logisitic Regression Using SAS: theory and application, Second Edition. Cary, NC: SAS Institute Inc.

Alves, J. G., Cisneiros, R. M., Dutra, L. P., \& Pinto, R. A. (2012). Perinatal characteristics among early (10-14 years old) and late (15-19 years old) pregnant adolescents. BMC Research Notes, 5(1), 531.

Anuradha, R. K., Sathyavathi, R. B., Reddy, T. M., Hemalatha, R., Sudhakar, G., Geetha, P., \& Reddy, K. K. (2015). Effect of social and environmental determinants on overweight and obesity prevalence among adolescent school children. Indian Journal of Endocrinology \& Metabolism, 19(2), 283-287. doi:10.4103/2230-8210.131765

Ary, D. V., Tildesley, E., Hops, H., \& Andrews, J. (1993). The influence of parent, sibling, and peer modeling and attitudes on adolescent use of alcohol. International Journal of the Addictions, 28(9), 853-880.

Aseltine, R. H., Schilling, E. A., James, A., Glanovsky, J. L., \& Jacobs, D. (2009). Age variability in the association between heavy episodic drinking and adolescent suicide attempts: findings from a large-scale, school-based screening program. Journal of the American Academy of Child \& Adolescent Psychiatry, 48(3), 262-270.

Baker, J. L., Olsen, L. W., \& Sørensen, T. I. (2007). Childhood body-mass index and the risk of coronary heart disease in adulthood. New England Journal of Medicine, 357(23), 23292337.

Bandura, A., \& McClelland, D. C. (1977). Social learning theory.

Barnes, G. M., Farrell, M. P., \& Banerjee, S. (1994). Family influences on alcohol abuse and other problem behaviors among black and white adolescents in a general population sample. Journal of Research on Adolescence, 4(2), 183-201.

Basen-Engquist, K., Edmundson, E. W., \& Parcel, G. S. (1996). Structure of health risk behavior among high school students. Journal of Consulting and Clinical Psychology, 64(4), 764775. doi:10.1037/0022-006X.64.4.764

Bhatia, S. K., \& Bhatia, S. C. (2007). Childhood and adolescent depression. American Family Physician, 75(1), 73-80.

Black, A. Y., Fleming, N. A., \& Rome, E. (2012). Pregnancy in adolescents. Adolescent Medicine: state of the art reviews, 23(1), 123-138, xi. 
Blum, R. W., Beuhring, T., Shew, M. L., Bearinger, L. H., Sieving, R. E., \& Resnick, M. D. (2000). The Effects of Race/Ethnicity, Income, and Family Structure on Adolescent Risk Behaviors. American Journal of Public Health, 90(12), 1879-1884.

Blum, R. W., \& Qureshi, F. (2011). Morbidity and mortality among adolescents and young adults in the United States. Baltimore, $M D$.

Boggess, S., Lindberg, L. D., Porter, L. (2000). Changes in risk-taking among high school students, 1991-1997: Evidence from the Yourth Risk Behavior Surveys. In Trends in the Wellbeing of America's Children and Youth 1999. U.S. Department of Health and Human Services, Washington, DC, pp. 475-488.

Booker, C., Gallaher, P., Unger, J., Ritt-Olson, A., \& Johnson, A. (2004). Stressful life events, smoking behavior, and intentions to smoke among a multiethnic sample of sixth graders. Ethnicity and Health, 9(4), 369-397.

Borowsky, I. W., Ireland, M., \& Resnick, M. D. (2001). Adolescent suicide attempts: risks and protectors. Pediatrics, 107(3), 485-493.

Bossarte, R. M., Simon, T. R., \& Swahn, M. H. (2008). Clustering of adolescent dating violence, peer violence, and suicidal behavior. Journal of Interpersonal Violence.

Brener, N. D., \& Collins, J. L. (1998). Co-occurrence of health-risk behaviors among adolescents in the United States. Journal of Adolescent Health, 22(3), 209-213.

Brener, N. D., McManus, T., Galuska, D. A., Lowry, R., \& Wechsler, H. (2002). Reliability and validity of self-reported height and weight among high school students. Journal of Adolescent Health, 32(4), 281-287.

Brook, D. W., Brook, J. S., Zhang, C., Cohen, P., \& Whiteman, M. (2002). Drug use and the risk of major depressive disorder, alcohol dependence, and substance use disorders. Archives of General Psychiatry, 59(11), 1039-1044.

Brown, L. K., Tolou-Shams, M., Lescano, C., Houck, C., Zeidman, J., Pugatch, D., \& Project SHIELD Study Group. (2006). Depressive symptoms as a predictor of sexual risk among African American adolescents and young adults. Journal of Adolescent Health, 39(3), 444-e1.

Carpenter, C. M., Wayne, G. F., Pauly, J. L., Koh, H. K., \& Connolly, G. N. (2005). New cigarette brands with flavors that appeal to youth: tobacco marketing strategies. Health Affairs, 24(6), 1601-1610.

Catalano, R. F., Fagan, A. A., Gavin, L. E., Greenberg, M. T., Irwin, C. E., Ross, D. A., \& Shek, D. T. (2012). Worldwide application of prevention science in adolescent health. The Lancet, 379(9826), 1653-1664.

Centers for Disease Control and Prevention. (2014). Youth Risk Behavior Survey data available at www.cdc.gov/yrbs

Chapman, C., Laird, J., Ifill, N., \& KewalRamani, A. (2011). Trends in High School Dropout and Completion Rates in the United States: 1972-2009. Compendium Report. NCES 2012-006. National Center for Education Statistics.

Chen, X.-K., Wen, S. W., Fleming, N., Demissie, K., Rhoads, G. G., \& Walker, M. (2007). Teenage pregnancy and adverse birth outcomes: a large population based retrospective cohort study. International Journal of Epidemiology, 36(2), 368-373.

Collins, L. M., Schafer, J. L., \& Kam, C.-M. (2001). A comparison of inclusive and restrictive strategies in modern missing data procedures. Psychological Methods, 6(4), 330. 
Connell, C. M., Gilreath, T. D., \& Hansen, N. B. (2009). A Multiprocess Latent Class Analysis of the Co-Occurrence of Substance Use and Sexual Risk Behavior Among Adolescents. Journal of Studies on Alcohol \& Drugs, 70(6), 943-951.

Costa, F. (2005). Problem Behavior Theory - A Brief Overview. Retrieved from http://www.colorado.edu/ibs/jessor/pb_theory.html

Costello, D. M., Swendsen, J., Rose, J. S., \& Dierker, L. C. (2008). Risk and protective factors associated with trajectories of depressed mood from adolescence to early adulthood. Journal of Consulting and Clinical Psychology, 76(2), 173.

Crow, S., Eisenberg, M. E., Story, M., \& Neumark-Sztainer, D. (2006). Psychosocial and behavioral correlates of dieting among overweight and non-overweight adolescents. Journal of Adolescent Health, 38(5), 569-574.

de la Haye, K., D'Amico, E. J., Miles, J. N. V., Ewing, B., \& Tucker, J. S. (2014). Covariance among Multiple Health Risk Behaviors in Adolescents. PLoS ONE, 9(5), 1-6.

DiClemente, R. J., Hansen, W. B., \& Ponton, L. E. (1996). Adolescents at risk. In Handbook of Adolescent Health Risk Behavior (pp. 1-4). Springer US.

Donovan, J. E. (1996). Problem behavior theory and the explanation of adolescent marijuana use. Journal of Drug Issues, 26(2), 379-404.

Donovan, J. E., Jessor, R., \& Costa, F. M. (1999). Adolescent problem drinking: stability of psychosocial and behavioral correlates across a generation. Journal of Studies on Alcohol, 60(3), 352-361.

Dukarm, C. P., Byrd, R. S., Auinger, P., \& Weitzman, M. (1996). Illicit substance use, gender, and the risk of violent behavior among adolescents. Archives of Pediatrics \& Adolescent Medicine, 150(8), 797-801.

Duke, N. N., \& Borowsky, I. W. (2015). Youth Violence Prevention and Safety: Opportunities for Health Care Providers. Pediatric Clinics of North America.

Eaton, D. K., Kann, L., Kinchen, S., Shanklin, S., Ross, J., Hawkins, J., . . Chyen, D. (2008). Youth risk behavior surveillance_-United States, 2007. MMWR Surveillance Summaries, 57(4), 1-131.

Ebbeling, C. B., Pawlak, D. B., \& Ludwig, D. S. (2002). Childhood obesity: public-health crisis, common sense cure. The Lancet, 360(9331), 473-482.

Farhat, T., Iannotti, R. J., \& Simons-Morton, B. G. (2010). Overweight, Obesity, Youth, and Health-Risk Behaviors. American Journal of Preventive Medicine, 38(3), 258-267. doi:10.1016/j.amepre.2009.10.038

Farrell, A. D., Danish, S. J., \& Howard, C. W. (1992). Relationship between drug use and other problem behaviors in urban adolescents. Journal of Consulting and Clinical Psychology, $60(5), 705$.

Fergusson, D. M., \& Lynskey, M. T. (1996). Alcohol misuse and adolescent sexual behaviors and risk taking. Pediatrics, 98(1), 91-96.

Fleming, N. A., Tu, X., \& Black, A. Y. (2012). Improved obstetrical outcomes for adolescents in a community-based outreach program: A matched cohort study. Journal of Obstetrics \& Gynaecology Canada, 34(12), 1134-1140.

Forman-Hoffman, V. (2004). High prevalence of abnormal eating and weight control practices among US high-school students. Eating behaviors, 5(4), 325-336.

Garrison, C. Z., McKeown, R. E., Valois, R. F., \& Vincent, M. L. (1993). Aggression, substance use, and suicidal behaviors in high school students. American Journal of Public Health, 83(2), 179-184. 
Gillmore, M. R., Butler, S. S., Lohr, M. J., \& Gilchrist, L. (1992). Substance use and other factors associated with risky sexual behavior among pregnant adolescents. Family Planning Perspectives, 255-268.

Gillmore, M. R., Hawkins, J. D., Catalano, R. F., Day, L. E., Moore, M., \& Abbott, R. (1991). Structure of problem behaviors in preadolescence. Journal of Consulting and Clinical Psychology, 59(4), 499.

Glanz, K., \& Bishop, D. B. (2010). The role of behavioral science theory in development and implementation of public health interventions. Annual Review of Public Health, 31, 399418.

Glanz, K., Rimer, B. K., \& Viswanath, K. (2008). Health Behavior and Health Education: theory, research, and practice: John Wiley \& Sons.

Goldschmidt, A. B., Jones, M., Manwaring, J. L., Luce, K. H., Osborne, M. I., Cunning, D., . . . Taylor, C. B. (2008). The clinical significance of loss of control over eating in overweight adolescents. International Journal of Eating Disorders, 41(2), 153-158.

Gould, M. S., Greenberg, T., Velting, D. M., \& Shaffer, D. (2003). Youth suicide risk and preventive interventions: a review of the past 10 years. Journal of the American Academy of Child \& Adolescent Psychiatry, 42(4), 386-405.

Green, B. E., \& Ritter, C. (2000). Marijuana use and depression. Journal of Health and Social Behavior, 40-49.

Groves, S. A., Stanley, B. H., \& Sher, L. (2007). Ethnicity and the relationship between adolescent alcohol use and suicidal behavior. International Journal of Adolescent Medicine and Health, 19(1), 19-26.

Guest, J., Bilgin, A., Pearce, R., Baines, S., Zeuschner, C., Rossignol-Grant, L., . . Grant, R. (2010). Evidence for under-nutrition in adolescent females using routine dieting practices. This article was originally published as: Guest, J., Bilgin, A., Pearce, R., Baines, S., Zeuschner, C., Le Rossignol-Grant, C., et al.(2010). Evidence for undernutrition in adolescent females using routine dieting practices. Asia Pacific Journal of Clinical Nutrition, 19 (4), 526-533. Asia Pacific Journal of Clinical Nutrition is published by HEC Press and may be accessed here.

Hackman, C. L., \& Knowlden, A. P. (2014). Theory of reasoned action and theory of planned behavior-based dietary interventions in adolescents and young adults: a systematic review. Adolescent Health, Medicine and Therapeutics, 5, 101.

Hagenaars J.A. \& McCutcheon, A.L. (2009). Applied Latent Class Analysis. Cambridge University Press.

Hale, D. R., Fitzgerald-Yau, N., \& Viner, R. M. (2014). A systematic review of effective interventions for reducing multiple health risk behaviors in adolescence. American Journal of Public Health, 104(5), e19-e41.

Hallfors, D. D., Waller, M. W., Ford, C. A., Halpern, C. T., Brodish, P. H., \& Iritani, B. (2004). Adolescent depression and suicide risk: association with sex and drug behavior. American Journal of Preventive Medicine, 27(3), 224-231.

Hamilton, B. E., Martin, J. A., \& Ventura, S. J. (2010). Births: Preliminary data for 2008. National Vital Statistics Reports, 58(16), 1-18.

Hawkins, J. D., Catalano, R. F., \& Miller, J. Y. (1992). Risk and protective factors for alcohol and other drug problems in adolescence and early adulthood: implications for substance abuse prevention. Psychological Bulletin, 112(1), 64.

Helzer, J. E., \& Pryzbeck, T. R. (1988). The co-occurrence of alcoholism with other psychiatric 
disorders in the general population and its impact on treatment. Journal of Studies on Alcohol, 49(3), 219-224.

Horn, K., Dino, G., Kalsekar, I., \& Mody, R. (2005). The impact of Not on Tobacco on teen smoking cessation end-of-program evaluation results, 1998 to 2003. Journal of Adolescent Research, 20(6), 640-661.

Jelalian, E., Alday, S., Spirito, A., Rasile, D., \& Nobile, C. (2000). Adolescent motor vehicle crashes: the relationship between behavioral factors and self-reported injury. Journal of Adolescent Health, 27(2), 84-93.

Jemmott III, J. B., Heeren, G. A., Ngwane, Z., Hewitt, N., Jemmott, L. S., Shell, R., \& O'leary, A. (2007). Theory of planned behaviour predictors of intention to use condoms among Xhosa adolescents in South Africa. AIDS Care, 19(5), 677-684.

Jessor, R. (1991). Risk behavior in adolescence: a psychosocial framework for understanding and action. Journal of Adolescent Health, 12(8), 597-605.

Jessor, R. (1992). Risk behavior in adolescence: A psychosocial framework for understanding and action. Developmental Review, 12(4), 374-390.

Jessor, R., Chase, J. A., \& Donovan, J. E. (1980). Psychosocial correlates of marijuana use and problem drinking in a national sample of adolescents. American Journal of Public Health, 70(6), 604-613.

Jessor, R., \& Jessor, S. L. (1977). Problem behavior and psychosocial development: A longitudinal study of youth.

Jessor, R., Van Den Bos, J., Vanderryn, J., Costa, F. M., \& Turbin, M. S. (1995). Protective factors in adolescent problem behavior: Moderator effects and developmental change. Developmental Psychology, 31(6), 923.

Jiang, Y., Perry, D. K., \& Hesser, J. E. (2010). Suicide Patterns and Association With Predictors Among Rhode Island Public High School Students: A Latent Class Analysis. American Journal of Public Health, 100(9), 1701-1707. doi:10.2105/AJPH.2009.183483

Johnson, N. B., Hayes, L. D., Brown, K., Hoo, E. C., \& Ethier, K. A. (2014). CDC National Health Report: leading causes of morbidity and mortality and associated behavioral risk and protective factors-United States, 2005-2013.MMWR Surveillance Summaries, 63(Suppl 4), 3-27.

Kaltiala-Heino, R., Rimpelä, M., Marttunen, M., Rimpelä, A., \& Rantanen, P. (1999). Bullying, depression, and suicidal ideation in Finnish adolescents: school survey. British Medical Journal, 319(7206), 348-351.

Kandel, D. B. (1985). On processes of peer influences in adolescent drug use: A developmental perspective. Advances in Alcohol \& Substance Abuse, 4(3-4), 139-162.

Kann, L., Kinchen, S., Shanklin, S. L., Flint, K. H., Hawkins, J., Harris, W. A., . . Zaza, S. (2014). Youth Risk Behavior Surveillance — United States, 2013. MMWR Surveillance Summaries, 63(4), 1-170.

Kessler, R. C., Nelson, C. B., McGonagle, K. A., Edlund, M. J., Frank, R. G., \& Leaf, P. J.

(1996). The epidemiology of co-occurring addictive and mental disorders: implications for prevention and service utilization. American Journal of Orthopsychiatry, 66(1), 17

Khan, M. R., Berger, A. T., Wells, B. E., \& Cleland, C. M. (2012). Longitudinal associations between adolescent alcohol use and adulthood sexual risk behavior and sexually transmitted infection in the United States: Assessment of differences by race. American Journal of Public Health, 102(5), 867-876. 
Kilpatrick, M., Ohannessian, C., \& Bartholomew, J. B. (1999). Adolescent weight management and perceptions: an analysis of the National Longitudinal Study of Adolescent Health. Journal of School Health, 69(4), 148-152.

Kim, C., Lesage, A., Seguin, M., Chawky, N., Vanier, C., Lipp, O., \& Turecki, G. (2003). Patterns of co-morbidity in male suicide completers. Psychological Medicine, 33(07), 1299-1309.

Kuther, T. L., \& Higgins-D'Alessandro, A. (2003). Attitudinal and normative predictors of alcohol use by older adolescents and young adults. Journal of Drug Education, 33(1), 7190.

Lammers, C., Ireland, M., Resnick, M., \& Blum, R. (2000). Influences on adolescents' decision to postpone onset of sexual intercourse: A survival analysis of virginity among youths aged 13 to 18 years. Journal of Adolescent Health, 26(1), 42-48.

Lindberg, L. D., Boggess, S., and Williams, S. (2000). Multiple threats: The co-occurrence of teen health risk-behaviors. In Trends in the Wellbeing of America's Children and Youth 1999. U.S. Department of Health and Human Services, Washington, DC, pp. 489-504.

López-Guimerà, G., Neumark-Sztainer, D., Hannan, P., Fauquet, J., Loth, K., \& SánchezCarracedo, D. (2013). Unhealthy Weight-control Behaviours, Dieting and Weight Status: A Cross-cultural Comparison between North American and Spanish Adolescents. European Eating Disorders Review, 21(4), 276-283.

Lormand, D. K., Markham, C. M., Peskin, M. F., Byrd, T. L., Addy, R. C., Baumler, E., \& Tortolero, S. R. (2013). Dating violence among urban, minority, middle school youth and associated sexual risk behaviors and substance use. Journal of School Health, 83(6), 415421.

Lowry, R., Galuska, D. A., Fulton, J. E., Wechsler, H., \& Kann, L. (2002). Weight management goals and practices among US high school students: associations with physical activity, diet, and smoking. Journal of Adolescent Health, 31(2), 133-144.

Ludwig, D. S. (2007). Childhood obesity - the shape of things to come. New England Journal of Medicine, 357(23), 2325-2327.

Lytle, L., \& Achterberg, C. (1995). Changing the diet of America's children: what works and why? Journal of Nutrition Education, 27(5), 250-260.

Ma, J., Betts, N. M., \& Hampl, J. S. (2000). Clustering of lifestyle behaviors: the relationship between cigarette smoking, alcohol consumption, and dietary intake. American Journal of Health Promotion, 15(2), 107-117.

MacArthur, G. J., Smith, M. C., Melotti, R., Heron, J., Macleod, J., Hickman, M., ... \& Lewis, G. (2012). Patterns of alcohol use and multiple risk behaviour by gender during early and late adolescence: the ALSPAC cohort. Journal of Public Health, 34(suppl 1), i20-i30.

Mahfouz, A. A., El-Said, M. M., Al-Erian, R. A., \& Hamid, A. M. (1995). Teenage pregnancy: are teenagers a high risk group? European Journal of Obstetrics \& Gynecology and Reproductive Biology, 59(1), 17-20.

Marshall, S. J., Biddle, S. J., Gorely, T., Cameron, N., \& Murdey, I. (2004). Relationships between media use, body fatness and physical activity in children and youth: a metaanalysis. International Journal of Obesity, 28(10), 1238-1246.

Martini, S., Wagner, F. A., \& Anthony, J. C. (2002). The association of tobacco smoking and depression in adolescence: evidence from the United States. Substance Use \& Misuse, 37(14), 1853-1867.Oesterle 2004 
McCave, E. L., \& Shiflet, A. (2010). A retrospective study of teen pregnancy in West Virginia from 2003-2008: a descriptive analysis. Contemporary Rural Social Work, 2, 24-31.

McCutcheon, A. L. (1987). Latent class analysis (Sage University Paper Series on Quantitative Applications in the Social Sciences, No. 07-064). Newbury Park, CA: Sage.

Minino, A. M. "Mortality among teenagers aged 12-29 years: United States, 1999-2006 (Data Brief No. 37)." Atlanta, GA: National Center for Health Statistics (2010).

Minnix, J. A., Blalock, J. A., Marani, S., Prokhorov, A. V., \& Cinciripini, P. M. (2011). Selfefficacy mediates the effect of depression on smoking susceptibility in adolescents. Nicotine \& Tobacco Research, 13(8), 699-705.

Mishna, F., Khoury-Kassabri, M., Gadalla, T., \& Daciuk, J. (2012). Risk factors for involvement in cyber bullying: Victims, bullies and bully-victims.Children and Youth Services Review, 34(1), 63-70.

Morrell, Cohen, and McChargue, 201 Morrell, H. E., Cohen, L. M., \& McChargue, D. E. (2010). Depression vulnerability predicts cigarette smoking among college students: Gender and negative reinforcement expectancies as contributing factors. Addictive Behaviors, 35(6), 607-611.

Must, A., Spadano, J., Coakley, E. H., Field, A. E., Colditz, G., \& Dietz, W. H. (1999). The disease burden associated with overweight and obesity. Journal of American Medical Association, 282(16), 1523-1529.

Nansel, T. R., Overpeck, M., Pilla, R. S., Ruan, W. J., Simons-Morton, B., \& Scheidt, P. (2001). Bullying behaviors among US youth: Prevalence and association with psychosocial adjustment. Journal of American Medical Association, 285(16), 2094-2100.

Neumark-Sztainer, D. (2007). Addressing the Spectrum of Adolescent Weight-Related Problems: Engaging Parents and Communities. Prevention Researcher, 14(3), 11-14.

Newcomb, M. E., Birkett, M., Corliss, H. L., \& Mustanski, B. (2014). Sexual orientation, gender, and racial differences in illicit drug use in a sample of US high school students. American Journal of Public Health, 104(2), 304-310.

Nezami, E., Unger, J., Tan, S., Mahaffey, C., Ritt-Olson, A., Sussman, S., ... \& Johnson, C. A. (2005). The influence of depressive symptoms on experimental smoking and intention to smoke in a diverse youth sample. Nicotine \& Tobacco Research, 7(2), 243-248.

Nylund, K. L., Asparouhov, T., \& Muthén, B. O. (2007). Deciding on the number of classes in latent class analysis and growth mixture modeling: A Monte Carlo simulation study. Structural Equation Modeling, 14(4), 535-569.

Ogden, C. L., Carroll, M. D., Kit, B. K., \& Flegal, K. M. (2014). Prevalence of childhood and adult obesity in the United States, 2011-2012. Journal of American Medical Association, 311(8), 806-814.

Osgood, D., \& Wilson, J. (1991). Covariation among health-compromising behaviors in adolescence. Paper presented at the A background paper for the Adolescent Health Project of the US Congress, Office of Technoloogy Assessment. Springfield, VA: National Technical Information Service.

Painter, J. E., Borba, C. P., Hynes, M., Mays, D., \& Glanz, K. (2008). The use of theory in health behavior research from 2000 to 2005: a systematic review. Annals of Behavioral Medicine, 35(3), 358-362.

Park, H. S., Schepp, K. G., Jang, E. H., \& Koo, H. Y. (2006). Predictors of suicidal ideation among high school students by gender in South Korea. Journal of School Health, 76(5), 181-188. 
Park, M., Falconer, C., Viner, R., \& Kinra, S. (2012). The impact of childhood obesity on morbidity and mortality in adulthood: a systematic review. Obesity Reviews, 13(11), 9851000.

Park, S., Blanck H. M., Sherry, B., Brener, N., \& O'Toole, T. (2012). Factors associated with sugar-sweetened beverage intake among United States high school students. Journal of Nutrition, 142(2), 306-312.

Park, M. J., Scott, J. T., Adams, S. H., Brindis, C. D., \& Irwin, C. E. (2014). Adolescent and young adult health in the United States in the past decade: little improvement and young adults remain worse off than adolescents. Journal of Adolescent Health, 55(1), 3-16.

Parks, S. E., Johnson, L. L., McDaniel, D. D., \& Gladden, M. (2014). Surveillance for violent deaths - national violent death reporting system, 16 states, 2010. MMWR Surveillance Summaries, 63(1), 1-33.

Patton, G. C., Carlin, J. B., Coffey, C., Wolfe, R., Hibbert, M., \& Bowes, G. (1998). Depression, anxiety, and smoking initiation: a prospective study over 3 years. American Journal of Public Health, 88(10), 1518-1522.

Pena, J. B., Matthieu, M. M., Zayas, L. H., Masyn, K. E., \& Caine, E. D. (2012). Co-occurring risk behaviors among White, Black, and Hispanic US high school adolescents with suicide attempts requiring medical attention, 1999-2007: implications for future prevention initiatives. Social Psychiatry \& Psychiatric Epidemiology, 47(1), 29-42. doi:10.1007/s00127-010-0322-z

Pesa, J. A., \& Turner, L. W. (2001). Fruit and vegetable intake and weight-control behaviors among US youth. American Journal of Health Behavior, 25(1), 3-9.

Petridou, E., Zavitsanos, X., Dessypris, N., Frangakis, C., Mandyla, M., Doxiadis, S., \& Trichopoulos, D. (1997). Adolescents in high-risk trajectory: clustering of risky behavior and the origins of socioeconomic health differentials. Preventive Medicine, 26(2), 215219.

Pickett, L. L., Ginsburg, H. J., Mendez, R. V., Lim, D. E., Blankenship, K. R., Foster, L. E., . . . Sheffield, S. B. (2012). Ajzen's theory of planned behavior as it relates to eating disorders and body satisfaction. North American Journal of Psychology, 14(2), 339.

Potthoff, S. J., Bearinger, L. H., Skay, C. L., Cassuto, N., Blum, R. W., \& Resnick, M. D. (1998). Dimensions of risk behaviors among American Indian youth. Archives of Pediatrics \& Adolescent Medicine, 152(2), 157-163.

Prochaska, J. J., Hall, S. M., Humfleet, G., Muňoz, R. F., Reus, V., Gorecki, J., \& Hu, D. (2008). Physical activity as a strategy for maintaining tobacco abstinence: a randomized trial. Preventive Medicine, 47(2), 215-220.

Regier, D. A., Farmer, M. E., Rae, D. S., Locke, B. Z., Keith, S. J., Judd, L. L., \& Goodwin, F. K. (1990). Comorbidity of mental disorders with alcohol and other drug abuse: results from the Epidemiologic Catchment Area (ECA) study. Journal of American Medical Association, 264(19), 2511-2518.

Reilly, J., \& Kelly, J. (2011). Long-term impact of overweight and obesity in childhood and adolescence on morbidity and premature mortality in adulthood: systematic review. International Journal of Obesity, 35(7), 891-898.

Reilly, J. J., Methven, E., McDowell, Z. C., Hacking, B., Alexander, D., Stewart, L., \& Kelnar, C. J. (2003). Health consequences of obesity. Archives of Disease in Childhood, 88(9), 748-752. 
Resnick, M. D., Bearman, P. S., Blum, R. W., Bauman, K. E., Harris, K. M., Jones, J., .. . Shew, M. (1997). Protecting adolescents from harm: findings from the National Longitudinal Study on Adolescent Health. Journal of American Medical Association, 278(10), 823832.

Resnicow, K., Ross-Gaddy, D., \& Vaughan, R. D. (1995). Structure of problem and positive behaviors in African American youths. Journal of Consulting and Clinical Psychology, 63(4), 594.

Rogers, M. M., Peoples-Sheps, M. D., \& Suchindran, C. (1996). Impact of a social support program on teenage prenatal care use and pregnancy outcomes. Journal of Adolescent Health, 19(2), 132-140.

Sawyer, S. M., Afifi, R. A., Bearinger, L. H., Blakemore, S.-J., Dick, B., Ezeh, A. C., \& Patton, G. C. (2012). Adolescence: a foundation for future health. The Lancet, 379(9826), 16301640.

Shrier, L. A., Emans, S. J., Woods, E. R., \& DuRant, R. H. (1997). The association of sexual risk behaviors and problem drug behaviors in high school students. Journal of Adolescent Health, 20(5), 377-383.

Silverman, J. G., Raj, A., Mucci, L. A., \& Hathaway, J. E. (2001). Dating violence against adolescent girls and associated substance use, unhealthy weight control, sexual risk behavior, pregnancy, and suicidality. Journal of American Medical Association, 286(5), 572-579.

Singh, A. S., Mulder, C., Twisk, J. W., Van Mechelen, W., \& Chinapaw, M. J. (2008). Tracking of childhood overweight into adulthood: a systematic review of the literature. Obesity Reviews, 9(5), 474-488.

Sleet, D. A., Ballesteros, M. F., \& Borse, N. N. (2010). A Review of Unintentional Injuries in Adolescents*. Annual Review of Public Health, 31, 195-212.

Smith, T. M. E., \& Sallee, D. N. (2008). Adolescents and the co-occurrence of the early onset of sexual intercourse and drug use risk behaviors. American Journal of Health Studies, 23(2).

Spriggs, A. L., Iannotti, R. J., Nansel, T. R., \& Haynie, D. L. (2007). Adolescent bullying involvement and perceived family, peer and school relations: Commonalities and differences across race/ethnicity. Journal of Adolescent Health, 41(3), 283-293.

Steuber, T. L., \& Danner, F. (2006). Adolescent smoking and depression: which comes first?. Addictive behaviors, 31(1), 133-136.

Stokes, M. E., Davis, C. S., \& Koch, G. G. (2012). Categorical data analysis using SAS. SAS institute.

Strachman, A., Impett, E. A., Henson, J. M., \& Pentz, M. A. (2009). Early adolescent alcohol use and sexual experience by emerging adulthood: a 10-year longitudinal investigation. Journal of Adolescent Health, 45(5), 478-482.

Strasburger, V. C. (2009). Children, adolescents and the media: what we know, what we don't know and what we need to find out (quickly!). Archives of Disease in Childhood, 94(9), 655-657.

Swahn, M. H., \& Bossarte, R. M. (2007). Gender, early alcohol use, and suicide ideation and attempts: findings from the 2005 youth risk behavior survey. Journal of Adolescent Health, 41(2), 175-181.

Tapert, S. F., Aarons, G. A., Sedlar, G. R., \& Brown, S. A. (2001). Adolescent substance use and sexual risk-taking behavior. Journal of Adolescent Health, 28(3), 181-189. 
Thompson, M. P., Kingree, J. B., \& Ho, C. h. (2006). Associations between delinquency and suicidal behaviors in a nationally representative sample of adolescents. Suicide and LifeThreatening Behavior, 36(1), 57-64.

Tildesley, E. A., Hops, H., Ary, D., \& Andrews, J. A. (1995). Multitrait-multimethod model of adolescent deviance, drug use, academic, and sexual behaviors. Journal of Psychopathology and Behavioral Assessment, 17(2), 185-215.

Umberson, D. (1992). Gender, marital status and the social control of health behavior. Social Science \& Medicine, 34(8), 907-917.

United States Department of Health and Human Services (2014). The health consequences of smoking--50 years of progress: A Report of the Surgeon General. Atlanta, GA Centers for Disease Control and Prevention. Retrieved from http://www.surgeongeneral.gov/library/reports/50-years-of-progress/exec-summary.pdf

U.S. Department of Health and Human Services and U.S. Department of Agriculture. 2015 2020 Dietary Guidelines for Americans. $8^{\text {th }}$ Edition. December 2015.

US Department of Health and Human Services. Healthy People 2010. $2^{\text {nd }}$ ed. With Understanding and Improving Health and Objectives for Improving Health. 2 vols. Washington, DC: US Government Printing Office; 2000.

US Department of Health and Human Services, Healthy People2020, US Department of Health and Human Services, Hyattsville, Md, USA, 2010.

Viner, R. M., Ozer, E. M., Denny, S., Marmot, M., Resnick, M., Fatusi, A., \& Currie, C. (2012). Adolescence and the social determinants of health. The Lancet, 379(9826), 1641-1652.

Wang, J., Iannotti, R. J., \& Nansel, T. R. (2009). School Bullying Among Adolescents in the United States: Physical, Verbal, Relational, and Cyber. Journal of Adolescent Health, 45(4), 368-375.

Wang, J., Nansel, T. R., \& Iannotti, R. J. (2011). Cyber and traditional bullying: Differential association with depression. Journal of Adolescent Health, 48(4), 415-417.

Wang, Y., \& Zhang, Q. (2006). Are American children and adolescents of low socioeconomic status at increased risk of obesity? Changes in the association between overweight and family income between 1971 and 2002. The American Journal of Clinical Nutrition, 84(4), 707-716.

Wayne, G. F., \& Connolly, G. (2002). How cigarette design can affect youth initiation into smoking: Camel cigarettes 1983-93. Tobacco Control, 11(suppl 1), i32-i39.

Weden, M. M., \& Zabin, L. S. (2005). Gender and ethnic differences in the co-occurrence of adolescent risk behaviors. Ethnicity and Health, 10(3), 213-234.

Wilcox, H. C. (2004). Epidemiological evidence on the link between drug use and suicidal behaviors among adolescents. The Canadian Child and Adolescent Psychiatry Review, 13(2), 27.

Williams, P. G., Holmbeck, G. N., \& Greenley, R. N. (2002). Adolescent health psychology. Journal of Consulting and Clinical Psychology, 70(3), 828.

Wills, T. A., Resko, J. A., Ainette, M. G., \& Mendoza, D. (2004). Smoking onset in adolescence: a person-centered analysis with time-varying predictors. Health Psychology, 23(2), 158.

World Bank, World Development Indicators. Adolescent Fertility Rate (births per 1,000 women ages 15-19) 2014-2015.

Yan, A. F., Chiu, Y. W., Stoesen, C. A., \& Wang, M. Q. (2007). STD-/HIV-related sexual risk behaviors and substance use among US rural adolescents. Journal of the National Medical Association, 99(12), 1386. 
Zuckerman, M. (1984). Sensation seeking: A comparative approach to a human trait. Behavioral and Brain Sciences, 7(03), 413-434.

Zweig, J. M., Lindberg, L. D., \& McGinley, K. A. (2001). Adolescent health risk profiles: The co-occurrence of health risks among females and males. Journal of Youth and Adolescence, 30(6), 707-728.

Zweig, J. M., Phillips, S. D., \& Lindberg, L. D. (2002). Predicting adolescent profiles of risk: looking beyond demographics. Journal of Adolescent Health, 31(4), 343-353.

\section{Chapter 3}

Basen-Engquist, K., Edmundson, E. W., \& Parcel, G. S. (1996). Structure of health risk behavior among high school students. Journal of Consulting and Clinical Psychology, 64(4), 764775.

Bhatia, S. K., \& Bhatia, S. C. (2007). Childhood and adolescent depression. American Family Physician, 75(1), 73-80.

Black, A. Y., Fleming, N. A., \& Rome, E. (2012). Pregnancy in adolescents. Adolescent Medicine: state of the art reviews, 23(1), 123-138, xi.

Blum, R. W., Beuhring, T., Shew, M. L., Bearinger, L. H., Sieving, R. E., \& Resnick, M. D. (2000). The Effects of Race/Ethnicity, Income, and Family Structure on Adolescent Risk Behaviors. American Journal of Public Health, 90(12), 1879-1884.

Blum, R. W., \& Qureshi, F. (2011). Morbidity and mortality among adolescents and young adults in the United States. Baltimore, MD.

Boggess, S., Lindberg, L. D., Porter, L. (2000). Changes in risk-taking among high school students, 1991-1997: Evidence from the Youth Risk Behavior Surveys. In Trends in the Wellbeing of America's Children and Youth 1999. U.S. Department of Health and Human Services, Washington, DC, pp. 475-488.

Brener, N. D., \& Collins, J. L. (1998). Co-occurrence of health-risk behaviors among adolescents in the United States. Journal of Adolescent Health, 22(3), 209-213.

Brener, N. D., McManus, T., Galuska, D. A., Lowry, R., \& Wechsler, H. (2003). Reliability and validity of self-reported height and weight among high school students. Journal of Adolescent Health, 32(4), 281-287.

Catalano, R. F., Fagan, A. A., Gavin, L. E., Greenberg, M. T., Irwin, C. E., Ross, D. A., \& Shek, D. T. (2012). Worldwide application of prevention science in adolescent health. The Lancet, 379(9826), 1653-1664.

Collins, L. M., Schafer, J. L., \& Kam, C.-M. (2001). A comparison of inclusive and restrictive strategies in modern missing data procedures. Psychological methods, 6(4), 330.

Connell, C. M., Gilreath, T. D., \& Hansen, N. B. (2009). A Multiprocess Latent Class Analysis of the Co-Occurrence of Substance Use and Sexual Risk Behavior Among Adolescents. Journal of Studies on Alcohol \& Drugs, 70(6), 943-951.

Farhat, T., Iannotti, R. J., \& Simons-Morton, B. G. (2010). Overweight, Obesity, Youth, and Health-Risk Behaviors. American Journal of Preventive Medicine, 38(3), 258-267.

Farrell, A. D., Danish, S. J., \& Howard, C. W. (1992). Relationship between drug use and other problem behaviors in urban adolescents. Journal of Consulting and Clinical Psychology, 60(5), 705.

Fergusson, D. M., \& Lynskey, M. T. (1996). Alcohol misuse and adolescent sexual behaviors and risk taking. Pediatrics, 98(1), 91-96.

Green, B. E., \& Ritter, C. (2000). Marijuana use and depression. Journal of Health and Social 
Behavior, 40-49.

Hamilton, B. E., Martin, J. A., \& Ventura, S. J. (2010). Births: Preliminary data for 2008. National Vital Statistics, 58(16), 1-18.

Jessor, R. (1991). Risk behavior in adolescence: a psychosocial framework for understanding and action. Journal of Adolescent Health, 12(8), 597-605.

Jessor, R. (1992). Risk behavior in adolescence: A psychosocial framework for understanding and action. Developmental Review, 12(4), 374-390.

Jessor, R., \& Jessor, S. L. (1977). Problem behavior and psychosocial development: A longitudinal study of youth.

Jessor, R., Van Den Bos, J., Vanderryn, J., Costa, F. M., \& Turbin, M. S. (1995). Protective factors in adolescent problem behavior: Moderator effects and developmental change. Developmental Psychology, 31(6), 923.

Kann, L., Kinchen, S., Shanklin, S. L., Flint, K. H., Hawkins, J., Harris, W. A., Zaza, S. (2014). Youth Risk Behavior Surveillance — United States, 2013. MMWR Surveillance Summaries, 63(4), 1-170.

Khan, M. R., Berger, A. T., Wells, B. E., \& Cleland, C. M. (2012). Longitudinal associations between adolescent alcohol use and adulthood sexual risk behavior and sexually transmitted infection in the United States: Assessment of differences by race. American Journal of Public Health, 102(5), 867-876.

Lindberg, L. D., Boggess, S., and Williams, S. (2000). Multiple threats: The co-occurrence of teen health risk-behaviors. In Trends in the Wellbeing of America's Children and Youth 1999. U.S. Department of Health and Human Services, Washington, DC, pp. 489-504.

Ma, J., Betts, N. M., \& Hampl, J. S. (2000). Clustering of lifestyle behaviors: the relationship between cigarette smoking, alcohol consumption, and dietary intake. American Journal of Health Promotion, 15(2), 107-117.

Martini, S., Wagner, F. A., \& Anthony, J. C. (2002). The association of tobacco smoking and depression in adolescence: evidence from the United States. Substance Use \& Misuse, 37(14), 1853-1867.Oesterle 2004

Minino, A. M. "Mortality among teenagers aged 12-29 years: United States, 1999-2006 (Data Brief No. 37)." Atlanta, GA: National Center for Health Statistics (2010).

Nansel, T. R., Overpeck, M., Pilla, R. S., Ruan, W. J., Simons-Morton, B., \& Scheidt, P. (2001). Bullying behaviors among US youth: Prevalence and association with psychosocial adjustment. Journal of American Medical Association, 285(16), 2094-2100.

Nylund, K. L., Asparouhov, T., \& Muthén, B. O. (2007). Deciding on the number of classes in latent class analysis and growth mixture modeling: A Monte Carlo simulation study. Structural Equation Modeling, 14(4), 535-569.

Pena, J. B., Matthieu, M. M., Zayas, L. H., Masyn, K. E., \& Caine, E. D. (2012). Co-occurring risk behaviors among White, Black, and Hispanic US high school adolescents with suicide attempts requiring medical attention, 1999-2007: implications for future prevention initiatives. Social Psychiatry \& Psychiatric Epidemiology, 47(1), 29-42.

Petrosino, A., Turpin-Petrosino, C., \& Buehler, J. (2003). Scared Straight and other juvenile awareness programs for preventing juvenile delinquency: A systematic review of the randomized experimental evidence. The Annals of the American Academy of Political and Social Science, 589(1), 41-62. 
Petrosino, A., Turpin-Petrosino, C., Hollis-Peel, M. E., \& Lavenberg, J. G. (2013). 'Scared Straight' and other juvenile awareness programs for preventing juvenile delinquency. The Cochrane Library.

Resnick, M. D., Bearman, P. S., Blum, R. W., Bauman, K. E., Harris, K. M., Jones, J., Shew, M. (1997). Protecting adolescents from harm: findings from the National Longitudinal Study on Adolescent Health. Journal of American Medical Association, 278(10), 823-832.

Resnicow, K., Ross-Gaddy, D., \& Vaughan, R. D. (1995). Structure of problem and positive behaviors in African American youths. Journal of Consulting and Clinical Psychology, 63(4), 594.

Smith, T. M. E., \& Sallee, D. N. (2008). Adolescents and the co-occurrence of the early onset of sexual intercourse and drug use risk behaviors. American Journal of Health Studies, 23(2).

United States Department of Health and Human Services (2014). The health consequences of smoking--50 years of progress: A report of the Surgeon General. Atlanta, GA Centers for Disease Control and Prevention.

Viner, R. M., Ozer, E. M., Denny, S., Marmot, M., Resnick, M., Fatusi, A., \& Currie, C. (2012). Adolescence and the social determinants of health. The Lancet, 379(9826), 1641-1652.

Weden, M. M., \& Zabin, L. S. (2005). Gender and ethnic differences in the co-occurrence of adolescent risk behaviors. Ethnicity and Health, 10(3), 213-234.

Zweig, J. M., Lindberg, L. D., \& McGinley, K. A. (2001). Adolescent health risk profiles: The co-occurrence of health risks among females and males. Journal of Youth and Adolescence, 30(6), 707-728.

\section{Chapter 4}

Anuradha, R. K., Sathyavathi, R. B., Reddy, T. M., Hemalatha, R., Sudhakar, G., Geetha, P., \& Reddy, K. K. (2015). Effect of social and environmental determinants on overweight and obesity prevalence among adolescent school children. Indian Journal of Endocrinology \& Metabolism, 19(2), 283-287.

Baker, J. L., Olsen, L. W., \& Sørensen, T. I. (2007). Childhood body-mass index and the risk of coronary heart disease in adulthood. New England Journal of Medicine, 357(23), 23292337.

Booker, C., Gallaher, P., Unger, J., Ritt-Olson, A., \& Johnson, A. (2004). Stressful life events, smoking behavior, and intentions to smoke among a multiethnic sample of sixth graders. Ethnicity and Health, 9(4), 369-397.

Brener, N. D., McManus, T., Galuska, D. A., Lowry, R., \& Wechsler, H. (2003). Reliability and validity of self-reported height and weight among high school students. Journal of Adolescent Health, 32(4), 281-287.

Cawley, J, Markowitz S, Tauras J. (2004). Lighting up and slimming down: the effects of body weight and cigarette prices on adolescent smoking initiation. Journal of Health Economics, 23(2):293-311.

Ebbeling, C. B., Pawlak, D. B., \& Ludwig, D. S. (2002). Childhood obesity: public-health crisis, common sense cure. The Lancet, 360(9331), 473-482.

Everett. S., Corman, H., \& Reichman, N.E. (2013). Effects of Overweight on Risky Sexual Behavior of Adolescent Girls. Economic Inquiry, 51(1), 605-619.

Falkner, N. H., Neumark-Sztainer, D., Story, M., Jeffery, R. W., Beuhring, T., \& Resnick, M. D. 
(2001). Social, educational, and psychological correlates of weight status in adolescents. Obesity Research, 9(1), 32-42.

Fallon, E. M., Tanofsky-Kraff, M., Norman, A. C., McDuffie, J. R., Taylor, E. D., Cohen, M. L., ... \& Yanovski, J. A. (2005). Health-related quality of life in overweight and nonoverweight black and white adolescents. Pediatrics, 147(4), 443-450.

Farhat, T., Iannotti, R. J., \& Simons-Morton, B. G. (2010). Overweight, Obesity, Youth, and Health-Risk Behaviors. American Journal of Preventive Medicine, 38(3), 258-267.

Freedman, D. S., Kettel, L., Serdula, M.K., Dietz, W.H., Srinivasan, S.R., Berenson, G.S. (2005). The relation of childhood BMI to adult adiposity: the Bogalusa Heart Study. Pediatrics, 115:22-27.

Freedman, D. S., Khan, L. K., Serdula, M. K., Ogden, C. L., \& Dietz, W. H. (2006). Racial and ethnic differences in secular trends for childhood BMI, weight, and height. Obesity, 14(2), 301-308.

Gordon, L. P., Diaz, A., Soghomonian, C., Nucci-Sack, A. T., Weiss, J. M., Strickler, H. D., \& ... Ochner, C. N. (2016). Increased Body Mass Index Associated with Increased Risky Sexual Behaviors. Journal of Pediatric \& Adolescent Gynecology, 29(1), 42-47.

Graber, J. A., Seeley, J. R., Brooks-Gunn, J., \& Lewinsohn, P. M. (2004). Is pubertal timing associated with psychopathology in young adulthood?. Journal of the American Academy of Child \& Adolescent Psychiatry, 43(6), 718-726.

Griffiths, L.J., Wolke, D., Page, A.S., Horwood, J. P. (2006). Obesity and bullying: different effects for boys and girls. Archives of Disease in Childhood, 91(2):121-5.

Janssen, I., Craig, W.M., Boyce, W.F., Pickett, W. (2004). Associations between overweight and obesity with bullying behaviors in school-aged children. Pediatrics, 113(5):1187-94.

Lanza, S. T., \& Collins, L. M. (2002). Pubertal timing and the onset of substance use in females during early adolescence. Prevention Science, 3(1), 69-82.

Lowry, R., Robin, L., Kann, L., \& Galuska, D. A. (2014). Associations of Body Mass Index with Sexual Risk-Taking and Injection Drug Use among US High School Students. Journal of Obesity, 1-10.

Lowry, R., Galuska, D.A., Fulton, J.E., Wechsler, H., Kann, L. (2002). Weight management goals and practices among U.S. high school students: associations with physical activity, diet, and smoking. J Adolesc Health, 31(2):133- 44.

Must, A., \& Strauss, R. S. (1999). Risks and consequences of childhood and adolescent obesity. International Journal of Obesity \& Related Metabolic Disorders, 23.

Oesterle S, Hill KG, Hawkins JD, et al. (2004). Adolescent heavy episodic drinking trajectories and health in young adulthood. J Stud Alcohol, 65:204 -12.

Park, M., Falconer, C., Viner, R., \& Kinra, S. (2012). The impact of childhood obesity on morbidity and mortality in adulthood: a systematic review. Obesity Reviews, 13(11), 985-1000.

Pasch, K.E., Nelson, M.C., Lytle, L.A., Moe, S.G., Perry, C.L. (2008). Adoption of risk-related factors through early adolescence: associations with weight status and implications for causal mechanisms. Journal of Adolescent Health, 43(4):387-93.

Reilly, J., \& Kelly, J. (2011). Long-term impact of overweight and obesity in childhood and adolescence on morbidity and premature mortality in adulthood: systematic review. International Journal of Obesity, 35(7), 891-898.

Reilly, J. J., Methven, E., McDowell, Z. C., Hacking, B., Alexander, D., Stewart, L., \& Kelnar, 
C. J. (2003). Health consequences of obesity. Archives of Disease in Childhood, 88(9), 748-752.

Singh, A. S., Mulder, C., Twisk, J. W., Van Mechelen, W., \& Chinapaw, M. J. (2008). Tracking of childhood overweight into adulthood: a systematic review of the literature. Obesity Reviews, 9(5), 474-488.

Strauss, R. S., \& Pollack, H. A. (2003). Social marginalization of overweight children. Archives of Pediatrics \& Adolescent Medicine, 157(8), 746-752.

Strauss, R. S. and H. A. Pollack. 2001. "Epidemic Increase in Childhood Overweight, 19861998." Journal of American Medical Association, 286:2845-2848.

Styne, D. M. (2001). Childhood and adolescent obesity: prevalence and significance. Pediatric Clinics of North America, 48(4), 823-854.

US Department of Health and Human Services, Healthy People2020, US Department of Health and Human Services, Hyattsville, Md, USA, 2010, http://www.healthypeople.gov/2020/default.aspx

Wang, Y. (2002). Is obesity associated with early sexual maturation? A comparison of the association in American boys versus girls. Pediatrics, 110(5), 903-910.

Zeller, M. H., Reiter-Purtill, J., \& Ramey, C. (2008). Negative peer perceptions of obese children in the classroom environment. Obesity, 16(4), 755-762.

\section{Chapter 5}

Anthony, D., Baggott, R., Tanner, J., Jones, K., Evans, H., Perkins, G. \& Palmer H. (2012). Health, Lifestyle, belief and knowledge differences between two ethnic groups with specific reference to tobacco, diet and physical activity. Journal of Advanced Nursing, 68(11), 2496-2503.

Brener, N. D., McManus, T., Galuska, D. A., Lowry, R., \& Wechsler, H. (2003). Reliability and validity of self-reported height and weight among high school students. Journal of Adolescent Health, 32(4), 281-287.

Brener, N.D., Kann, L., Shanklin S. et al. (2013). "Methodology of the youth risk behavior surveillance system-2013," Morbidity and Mortality Weekly Report: Recommendations and Reports, vol. 62, no. 12, pp. 1-23, 2013.

Brener, N.D., Collins, J.L., Kann, L., Warren, C.W>, \& William, B.I. (1995). Reliability of the Youth Risk Behavior Survey Questionnaire. American Journal of Epidemiology, 141(6), 575-580.

Dino, G. A., Horn, K. A., Goldcamp, J., Maniar, S. D., Fernandes, A., \& Massey, C. J. (2001). Statewide demonstration of not on tobacco: a gender-sensitive teen smoking cessation program. The Journal of School Nursing,17(2), 90-97.

Dvorak, R. D., Del Gaizo, A. L., Engdahl, R. M., \& Eliason, C. J. (2009). Tobacco use and body mass index Mediated effects through physical inactivity. Journal of Health Psychology, 14(7), 919-923.

Farhat, T., Iannotti, R. J., \& Simons-Morton, B. G. (2010). Overweight, Obesity, Youth, and Health-Risk Behaviors. American Journal of Preventive Medicine, 38(3), 258-267.

Gidwani, P. P., Sobol, A., DeJong, W., Perrin, J. M., \& Gortmaker, S. L. (2002). Television viewing and initiation of smoking among youth. Pediatrics,110(3), 505-508.

Hancox, R.J., Milne, B.J., \& Poulton, R. (2004). Association between child and adolescent television viewing and adult health: a longitudinal birth cohort study. Lancet, 364:257-62. 
Ho “glund D, Samuelson G \& Mark A (1998): Food habits in Swedish adolescents in relation to socioeconomic conditions. European Journal of Clinical Nutrition. 52, 784-789

Horn, K., Dino, G., Branstetter, S. A., Zhang, J., Noerachmanto, N., Jarrett, T., \& Taylor, M. (2011). Effects of physical activity on teen smoking cessation. Pediatrics, 128(4), e801e811.Isralowitz RE \& Trostler N (1996): Substance use: toward an understanding of its relation to nutrition-related attitudes and behavior among Israeli high school youth. Journal of adolescent health, 19, 184-189.

Isralowitz, R. E., \& Trostler, N. (1996). Substance use: toward an understanding of its relation to nutrition-related attitudes and behavior among Israeli high school youth. Journal of Adolescent Health, 19(3), 184-189.

Johnson, J.G., Cohen, P., Smailes, E.M., Kasen, S., Brook, J.S. (2002). Television viewing and aggressive behavior during adolescence and adulthood. Science, 295(5564):2468-2471.

Kann, L., Kinchen, S., Shanklin, S. L., Flint, K. H., Hawkins, J., Harris, W. A., . . Zaza, S. (2014). Youth Risk Behavior Surveillance — United States, 2013. MMWR Surveillance Summaries, 63(4), 1-170.

Katzmarzyk, P. T., Church, T. S., Craig, C. L., \& Bouchard, C. (2009). Sitting time and mortality from all causes, cardiovascular disease, and cancer. Medicine and Science in Sports \& Exercise, 41(5), 998-1005.

Keski-Rahkonen, A., Kaprio, J., Rissanen, A., Virkkunen, M., \& Rose, R. J. (2003). Breakfast skipping and health-compromising behaviors in adolescents and adults. European Journal of Clinical Nutrition, 57(7), 842-853.

Korhonen, T., Kujala, U.M., Rose, R.J., Kaprio, J. (2009). Physical activity in adolescence as a predictor of alcohol and illicit drug use in early adulthood: a longitudinal populationbased twin study. Twin Research and Human Genetics, 12(3):261-8.

Kujala, U.M., Kaprio, J., Rose, R.J. (2007). Physical activity in adolescence and smoking in young adulthood: a prospective twin cohort study. Addiction. 2007;102(7):1151-7.

López-Guimerà, G., Neumark-Sztainer, D., Hannan, P., Fauquet, J., Loth, K., \& SánchezCarracedo, D. (2013). Unhealthy Weight-control Behaviours, Dieting and Weight Status: A Cross-cultural Comparison between North American and Spanish Adolescents. European Eating Disorders Review, 21(4), 276-283.

Lando, H. A., Thai, D. T., Murray, D. M., Robinson, L. A., Jeffery, R. W., Sherwood, N. E., \& Hennrikus, D. J. (1999). Age of initiation, smoking patterns, and risk in a population of working adults. Preventive Medicine, 29(6), 590-598.

Larson NI, Story M, Perry CL, Neumark-Sztainer D, Hannan PJ. (2007). Are diet and physical activity patterns related to cigarette smoking in adolescents? Findings from Project EAT. Preventing Chronic Disease, 4: 1-12.

Lowry, R., Galuska, D.A., Fulton, J.E., Wechsler, H., Kann, L. (2002). Weight management goals and practices among U.S. high school students: associations with physical activity, diet, and smoking. Journal of Adolescent Health, 31(2):133- 44.

Ludwig, D. S. (2007). Childhood obesity - the shape of things to come. New England Journal of Medicine, 357(23), 2325-2327.

Ma, J., Betts, N. M., \& Hampl, J. S. (2000). Clustering of lifestyle behaviors: the relationship between cigarette smoking, alcohol consumption, and dietary intake. American Journal of Health Promotion, 15(2), 107-117.

Moore, M.J., Werch, C.E. (2005). Sport and physical activity participation and substance use among adolescents. Journal of Adolescent Health, 36 (6):486-93. 
Owen, N., Healy, G. N., Matthews, C. E., \& Dunstan, D. W. (2010). Too much sitting: the population health science of sedentary behavior. Exercise and Sport Sciences Reviews, 38(3), 105.

Pate, R, Heath, G.W., Dowda, M., \& Trost, S.G. (1996). Associations between physical activity and other health behaviors in a representative sample of US adolescents. American Journal of Public Health, 86(11), 1577-1581.

Pearson, N., \& Biddle, S. J. (2011). Sedentary behavior and dietary intake in children, adolescents, and adults: a systematic review. American Journal of Preventive Medicine, 41(2), 178-188.

Respress, B. N., Morris, D. L., Gary, F. A., Lewin, L. C., \& Francis, S. A. (2013). Social Determinants of Adolescent Depression: An Examination of Racial Differences. Issues in Mental Health Nursing, 34(7), 539-549.

Rideout, V.J., Foehr, U.G., Roberts, D.F. (2010). Generation M2: media in the lives of 8- to 18year-olds. A Kaiser Family Foundation Study.

Strasburger, V.C., Jordan, A.B., Donnerstein, E. (2010). Health effects of media on children and adolescents. Pediatrics, 125(4):756-767

Sallis, J. F., Prochaska, J. J., \& Taylor, W. C. (2000). A review of correlates of physical activity of children and adolescents. Medicine and Science in Sports \& Exercise, 32(5), 963-975.

Salmon, J., Tremblay, M. S., Marshall, S. J., \& Hume, C. (2011). Health risks, correlates, and interventions to reduce sedentary behavior in young people. American Journal of Preventive Medicine, 41(2), 197-206.

Tremblay, M. S., LeBlanc, A. G., Kho, M. E., Saunders, T. J., Larouche, R., Colley, R. C., ... \& Gorber, S. C. (2011). Systematic review of sedentary behaviour and health indicators in school-aged children and youth. International Journal of Behavioral Nutrition and Physical Activity, 8(1), 1.

Troiano, R. P., Berrigan, D., Dodd, K. W., Masse, L. C., Tilert, T., \& McDowell, M. (2008). Physical activity in the United States measured by accelerometer. Medicine and Science in Sports \& Exercise, 40(1), 181.

Troped, P. J., Wiecha, J. L., Fragala, M. S., Matthews, C. E., Finkelstein, D. M., Kim, J., \& Peterson, K. E. (2007). Reliability and validity of YRBS physical activity items among middle school students. Medicine and Science in Sports \& Exercise, 39(3), 416-425.

U.S. Department of Health and Human Services and U.S. Department of Agriculture. 2015 2020 Dietary Guidelines for Americans. $8^{\text {th }}$ Edition. December 2015.

US Department of Health and Human Services. Healthy People 2010. $2^{\text {nd }}$ ed. With Understanding and Improving Health and Objectives for Improving Health. 2 vols. Washington, DC: US Government Printing Office; 2000.

US Department of Health and Human Services, Healthy People2020, US Department of Health and Human Services, Hyattsville, Md, USA, 2010, http://www.healthypeople.gov/2020/default.aspx

Yusuf, S., Hawken, S., Ounpuu, S., Dans, T., Avezum, A., Lanas, F., McQueen, M., Budaj, A., Pais, P., Varigos, J., Lisheng, L. (2004). Effect of potentially modifiable risk factors associated with myocardial infarction in 52 countries (the INTERHEART study): casecontrol study. Lancet, 364:937-952.

Wilson, D. B., Smith, B. N., Speizer, I. S., Bean, M. K., Mitchell, K. S., Uguy, L. S., \& Fries, E. A. (2005). Differences in food intake and exercise by smoking status in adolescents. Preventive Medicine, 40(6), 872-879. 
Wallace Jr, J. M., Bachman, J. G., O'Malley, P. M., Johnston, L. D., Schulenberg, J. E., \& Cooper, S. M. (2002). Tobacco, alcohol, and illicit drug use: racial and ethnic differences among US high school seniors, 1976-2000. Public Health Reports, 117(Suppl 1), S67.

Winnail, S.D., Valois, R.F., McKeown, R.E., Saunders, R.P., Pate, R.R. (1995). Relationship between physical activity level and cigarette, smokeless tobacco, and marijuana use among public high school adolescents. Journal of School Health, 65(10):438-42.

\section{Chapter 6}

Aseltine, R. H., Schilling, E. A., James, A., Glanovsky, J. L., \& Jacobs, D. (2009). Age variability in the association between heavy episodic drinking and adolescent suicide attempts: findings from a large-scale, school-based screening program. Journal of the American Academy of Child \& Adolescent Psychiatry, 48(3), 262-270.

Brener, N. D., McManus, T., Galuska, D. A., Lowry, R., \& Wechsler, H. (2003). Reliability and validity of self-reported height and weight among high school students. Journal of Adolescent Health, 32(4), 281-287.

Chapman, C., Laird, J., Ifill, N., \& KewalRamani, A. (2011). Trends in High School Dropout and Completion Rates in the United States: 1972-2009. Compendium Report. NCES 2012-006. National Center for Education Statistics.

Connell, C. M., Gilreath, T. D., \& Hansen, N. B. (2009). A Multiprocess Latent Class Analysis of the Co-Occurrence of Substance Use and Sexual Risk Behavior among Adolescents. Journal of Studies on Alcohol \& Drugs, 70(6), 943-951.

Dukarm, C. P., Byrd, R. S., Auinger, P., \& Weitzman, M. (1996). Illicit substance use, gender, and the risk of violent behavior among adolescents. Archives of Pediatrics \& Adolescent Medicine, 150(8), 797-801.

Helzer, J. E., \& Pryzbeck, T. R. (1988). The co-occurrence of alcoholism with other psychiatric disorders in the general population and its impact on treatment. Journal of Studies on Alcohol, 49(3), 219-224.

Ho"glund D, Samuelson G \& Mark A (1998): Food habits in Swedish adolescents in relation to socioeconomic conditions. European Journal of Clinical Nutrition, 52, 784-789.

Isralowitz, R. E., \& Trostler, N. (1996). Substance use: toward an understanding of its relation to nutrition-related attitudes and behavior among Israeli high school youth. Journal of Adolescent Health, 19(3), 184-189.

Katzmarzyk, P. T., Church, T. S., Craig, C. L., \& Bouchard, C. (2009). Sitting time and mortality from all causes, cardiovascular disease, and cancer. Medicine and Science in Sports \& Exercise, 41(5), 998-1005.

Keski-Rahkonen, A., Kaprio, J., Rissanen, A., Virkkunen, M., \& Rose, R. J. (2003). Breakfast skipping and health-compromising behaviors in adolescents and adults. European Journal of Clinical Nutrition, 57(7), 842-853.

Kessler, R. C., Nelson, C. B., McGonagle, K. A., Edlund, M. J., Frank, R. G., \& Leaf, P. J. (1996). The epidemiology of co-occurring addictive and mental disorders: implications for prevention and service utilization. American Journal of Orthopsychiatry, 66(1), 17.

Martini, S., Wagner, F. A., \& Anthony, J. C. (2002). The association of tobacco smoking and depression in adolescence: evidence from the United States. Substance Use \& Misuse, 37(14), 1853-1867.Oesterle 2004

Minnix, J. A., Blalock, J. A., Marani, S., Prokhorov, A. V., \& Cinciripini, P. M. (2011). Self- 
efficacy mediates the effect of depression on smoking susceptibility in adolescents. Nicotine \& Tobacco Research, 13(8), 699-705.

Ogden, C. L., Carroll, M. D., Kit, B. K., \& Flegal, K. M. (2014). Prevalence of childhood and adult obesity in the United States, 2011-2012. Jama, 311(8), 806-814.

Oesterle, S., Hill, K. G., Hawkins, J. D., Guo, J., Catalano, R. F., \& Abbott, R. D. (2004). Adolescent heavy episodic drinking trajectories and health in young adulthood. Journal of Studies on Alcohol, 65(2), 204.

Patton, G. C., Carlin, J. B., Coffey, C., Wolfe, R., Hibbert, M., \& Bowes, G. (1998). Depression, anxiety, and smoking initiation: a prospective study over 3 years. American Journal of Public Health, 88(10), 1518-1522.

Pena, J. B., Matthieu, M. M., Zayas, L. H., Masyn, K. E., \& Caine, E. D. (2012). Co-occurring risk behaviors among White, Black, and Hispanic US high school adolescents with suicide attempts requiring medical attention, 1999-2007: implications for future prevention initiatives. Social Psychiatry \& Psychiatric Epidemiology, 47(1), 29-42.

Regier, D. A., Farmer, M. E., Rae, D. S., Locke, B. Z., Keith, S. J., Judd, L. L., \& Goodwin, F. K. (1990). Comorbidity of mental disorders with alcohol and other drug abuse: results from the Epidemiologic Catchment Area (ECA) study. Journal of American Medical Association, 264(19), 2511-2518.

Rideout, V. J., Foehr, U. G., \& Roberts, D. F. (2010). Generation M [superscript 2]: Media in the Lives of 8-to 18-Year-Olds. Henry J. Kaiser Family Foundation.

Sallis, J. F., Prochaska, J. J., \& Taylor, W. C. (2000). A review of correlates of physical activity of children and adolescents. Medicine and Science in Sports \& Exercise, 32(5), 963-975.

Shrier, L. A., Emans, S. J., Woods, E. R., \& DuRant, R. H. (1997). The association of sexual risk behaviors and problem drug behaviors in high school students. Journal of Adolescent Health, 20(5), 377-383.

Steuber, T. L., \& Danner, F. (2006). Adolescent smoking and depression: which comes first?. Addictive Behaviors, 31(1), 133-136.

Strasburger, V. C. (2009). Children, adolescents and the media: what we know, what we don't know and what we need to find out (quickly!). Archives of Disease in Childhood, 94(9), 655-657.

Wilson, D. B., Smith, B. N., Speizer, I. S., Bean, M. K., Mitchell, K. S., Uguy, L. S., \& Fries, E. A. (2005). Differences in food intake and exercise by smoking status in adolescents. Preventive Medicine, 40(6), 872-879.

Zweig, J. M., Lindberg, L. D., \& McGinley, K. A. (2001). Adolescent health risk profiles: The co-occurrence of health risks among females and males. Journal of Youth and Adolescence, 30(6), 707-728. 


\section{Tables}

\section{Chapter 3}

Table 1. Demographic characteristics of adolescents in the 2013 national YRBSS $(\mathrm{N}=13,583)$.

\begin{tabular}{|c|c|c|}
\hline & Frequency & Weighted $\%$ \\
\hline \multicolumn{3}{|l|}{ Age } \\
\hline 12 years or younger & 26 & 0.2 \\
\hline 13 years old & 18 & 0.1 \\
\hline 14 years old & 1,368 & 10.0 \\
\hline 15 years old & 3,098 & 24.1 \\
\hline 16 years old & 3,203 & 25.3 \\
\hline 17 years old & 3,473 & 24.6 \\
\hline 18 years old & 2,320 & 15.6 \\
\hline \multicolumn{3}{|l|}{ Sex } \\
\hline Female & 6,621 & 50.0 \\
\hline Male & 6,950 & 50.0 \\
\hline \multicolumn{3}{|l|}{ Race/Ethnicity } \\
\hline American Indian/Alaska Native & 121 & 0.7 \\
\hline Asian & 491 & 3.0 \\
\hline Black or African American & 2,993 & 14.3 \\
\hline Native Hawaiian/Other PI & 135 & 0.8 \\
\hline White & 5,449 & 55.6 \\
\hline Hispanic/Latino & 1,734 & 10.4 \\
\hline Multiple - Hispanic & 1,661 & 10.7 \\
\hline Multiple - non-Hispanic & 681 & 4.4 \\
\hline \multicolumn{3}{|l|}{ Grade } \\
\hline $9^{\text {th }}$ & 3,588 & 27.3 \\
\hline $10^{\text {th }}$ & 3,152 & 25.7 \\
\hline $11^{\text {th }}$ & 3,184 & 23.8 \\
\hline $12^{\text {th }}$ & 3,557 & 23.1 \\
\hline
\end{tabular}


Table 2. Behavioral characteristics of adolescents in the 2013 national YRBSS

\begin{tabular}{|c|c|c|c|}
\hline & $\begin{array}{l}\text { Total Sample } \\
(N=13,571)\end{array}$ & $\begin{array}{l}\text { Female } \\
(N=6621)\end{array}$ & $\begin{array}{l}\text { Male } \\
(\mathrm{N}=6950)\end{array}$ \\
\hline Behavioral characteristics & $\mathrm{n}(\%)$ & $\mathrm{n}(\%)$ & $\mathrm{n}(\%)$ \\
\hline \multicolumn{4}{|l|}{ Tobacco Use } \\
\hline $\begin{array}{l}\text { Currently smoked cigarettes ( } 1 \text { or more days in the } \\
\text { past } 30 \text { days) }\end{array}$ & $1886(15.1)$ & $807(14.5)$ & $1079(15.6)$ \\
\hline $\begin{array}{l}\text { Smoked daily at least } 1 \text { cigarette every day/past } 30 \\
\text { days }\end{array}$ & $1039(8.3)$ & $430(8.2)$ & $609(8.4)$ \\
\hline $\begin{array}{l}\text { Currently used tobacco (cigarette use, current } \\
\text { smokeless tobacco use, or current cigar use) }\end{array}$ & $2666(21.1)$ & $997(17.0)$ & $1669(25.2)$ \\
\hline \multicolumn{4}{|l|}{ Marijuana use } \\
\hline $\begin{array}{l}\text { Currently used marijuana ( } 1+\text { days during the last } 30 \\
\text { days) }\end{array}$ & $3364(23.0)$ & $1498(21.6)$ & $1866(24.4)$ \\
\hline \multicolumn{4}{|l|}{ Alcohol use } \\
\hline $\begin{array}{l}\text { Currently drank alcohol (1+ days during the last } 30 \\
\text { days) }\end{array}$ & $4369(32.0)$ & $2180(32.6)$ & $2189(31.3)$ \\
\hline $\begin{array}{l}5+\text { Drinks in a row/one or more times in the past } 30 \\
\text { days }\end{array}$ & $2620(20.1)$ & $1189(19.1)$ & $1425(21.0)$ \\
\hline \multicolumn{4}{|l|}{ Other Drug Use (lifetime use) } \\
\hline cocaine & $746(5.5)$ & $271(4.4)$ & $475(6.5)$ \\
\hline $\begin{array}{l}\text { Sniffed glue, breathed content of aerosol spray cans, } \\
\text { inhaled paints or sprays to get high }\end{array}$ & $1175(8.8)$ & $622(9.8)$ & $553(7.7)$ \\
\hline Ecstasy & $935(6.4)$ & $368(5.5)$ & $567(7.4)$ \\
\hline *Prescription drug without a doctor's prescription & $2347(17.6)$ & $1069(17.1)$ & $1278(18.1)$ \\
\hline \multicolumn{4}{|l|}{ Unintentional injuries } \\
\hline Never or rarely wore bicycle helmet & $7687(57.7)$ & $3256(51.2)$ & $4431(64.1)$ \\
\hline Never or rarely wore seat belt & $1051(7.6)$ & $405(6.1)$ & $646(9.1)$ \\
\hline Rode with a driver who had been drinking alcohol & $3072(21.8)$ & $1538(22.3)$ & $1534(21.4)$ \\
\hline Drove when drinking alcohol & $759(5.9)$ & $253(4.4)$ & $506(7.5)$ \\
\hline Texted while driving a car or other vehicle & $3600(26.8)$ & $1422(23.6)$ & $1800(26.5)$ \\
\hline \multicolumn{4}{|l|}{ Violence } \\
\hline Carried a weapon & $2257(17.5)$ & $479(7.7)$ & $1778(27.2)$ \\
\hline Carried a weapon on school property & $627(5.2)$ & & \\
\hline Were in a physical fight & $3617(24.2)$ & $1385(18.9)$ & $2232(29.5)$ \\
\hline \multicolumn{4}{|l|}{ Bullying } \\
\hline Were bullied on school property & $2505(19.5)$ & $1472(23.6)$ & $1033(15.4)$ \\
\hline Were electronically bullied & $1878(14.7)$ & $1300(20.9)$ & $578(8.5)$ \\
\hline \multicolumn{4}{|l|}{ Sadness/hopelessness } \\
\hline $\begin{array}{l}\text { Felt sad or hopeless (almost every day for } 2 \text { or more } \\
\text { weeks in a row in past year) }\end{array}$ & $4084(29.7)$ & $2592(38.9)$ & $1492(20.6)$ \\
\hline \multicolumn{4}{|l|}{ Suicidal ideation } \\
\hline $\begin{array}{l}\text { Seriously considered attempting suicide during the } \\
\text { past year }\end{array}$ & $2259(17.0)$ & $1469(22.2)$ & $789(11.5)$ \\
\hline Made a plan about how they would attempt suicide & $1874(13.5)$ & $1175(16.8)$ & $699(10.2)$ \\
\hline \multicolumn{4}{|l|}{ Risky sexual behavior } \\
\hline $\begin{array}{l}\text { Had sexual intercourse with } 4+\text { persons (during their } \\
\text { life) }\end{array}$ & 2155 (14.3) & $806(12.8)$ & $1349(15.7)$ \\
\hline
\end{tabular}


Were currently sexually active (sexual intercourse with at least one person during the 3 months before the survey)

Did not use condom

Drank alcohol or used drugs before last sexual intercourse

Weight Status

Were overweight $\left(\geq 85^{\text {th }}\right.$ percentile of BMI)

Were obese $\left(\geq 95^{\text {th }}\right.$ percentile of BMI)

Unhealthy Weight Control

Did not eat for 24+ hours to lose weight or keep from gaining weight

\begin{tabular}{ccc}
$4663(32.4)$ & $2288(34.2)$ & $2375(30.7)$ \\
$1859(12.9)$ & $1061(17.9)$ & $798(10.2)$ \\
$1039(7.2)$ & $427(6.5)$ & $612(7.9)$ \\
$2064(16.6)$ & $1044(16.6)$ & $1020(16.5)$ \\
$1786(13.7)$ & $732(10.8)$ & $1054(16.5)$ \\
$1781(12.9)$ & $1218(18.5)$ & $563(7.3)$ \\
$684(4.9)$ & $431(6.5)$ & $253(3.3)$ \\
$558(4.3)$ & $402(6.4)$ & $156(2.1)$ \\
$686(4.9)$ & $288(3.9)$ & $398(5.9)$ \\
$941(6.5)$ & $401(5.6)$ & $540(7.3)$ \\
$2816(19.0)$ & $1816(25.1)$ & $1000(12.9)$ \\
$2588(19.3)$ & $1091(16.3)$ & $1493(21.7)$ \\
$1942(13.4)$ & $976(13.6)$ & $966(13.2)$ \\
$2171(14.9)$ & $1383(18.9)$ & $788(10.9)$ \\
$4777(31.8)$ & $2320(31.8)$ & $2457(31.9)$ \\
$5665(40.5)$ & $2742(39.8)$ & $2923(41.2)$ \\
\hline
\end{tabular}

Took diet pills, powders, or liquids

Vomited or took laxatives to lose weight or keep

from gaining weight

Poor dietary behavior

Did not eat fruit or drink 100\% fruit juices (during

the past 7 days before the survey)

Did not any vegetables (during the past 7 days before

the survey)

Did not drink milk (during the past 7 days before the survey)

Drank a can, bottle, or glass of soda $2+$ times per day

(during the past 7 days before the survey)

Did not eat breakfast (during the past 7 days before

the survey)

Physical inactivity and Sedentary Behavior

Physically active on 0 days past 7 days

217

Played video or computer games 3 or more hours/day

weighted percentages are reported for representativeness of data to the US high school students

*prescription drugs such as OxyContin, Percocet, Vicodin, Codeine, Adderall, Ritalin, or Xanax

Row percentages reported for female and male students 
Table 3. Fit statistic comparisons of latent classes of health risk behaviors among adolescents $(\mathrm{N}=13853)$

\begin{tabular}{lcccc}
\hline \multicolumn{5}{c}{ Deciding on the number of classes } \\
\hline Fit indices & 2 & 3 & 4 & 5 \\
\hline AIC & 386453.62 & 379290.56 & 372896.88 & 370556.20 \\
ABIC & 386805.05 & 379819.88 & 373604.09 & 371441.29 \\
BIC & 387062.46 & 380207.58 & 374122.09 & 372089.58 \\
TECH 11 LRT & 39695.50 & 7226.53 & 6459.12 & 2416.49 \\
p-value for k-1 & 0.0000 & 0.3283 & 0.4633 & 0.6380 \\
Entropy & 0.903 & 0.868 & 0.866 & 0.878 \\
\hline
\end{tabular}

Akaike Information Criterion, AIC

Bayesian Information Criterion BIC

Sample size Ajusted BIC, ABIC

Table 4. Fit statistic comparisons of latent classes of health risk behaviors among female adolescents $(\mathrm{N}=66210)$

\begin{tabular}{lcccccccc}
\hline \multicolumn{1}{c}{ Fit indices } & \multicolumn{1}{c}{ Deciding on the number of classes } \\
& 2 & 3 & 4 & 5 & \multicolumn{4}{c}{ \# of Classes for boys (n=6950) } \\
\hline AIC & 189109.67 & 185335.46 & 182444.13 & 180944.51 & 191644.53 & 188327.46 & 185415.95 & 184050.10 \\
ABIC & 189402.91 & 185777.13 & 183034.23 & 181683.04 & 191941.70 & 188775.04 & 186013.95 & 184798.53 \\
BIC & 189660.31 & 186164.81 & 183552.20 & 182331.31 & 192199.09 & 189162.73 & 186531.93 & 185446.79 \\
TECH 11 LRT & 18655.38 & 3845.55 & 2965.11 & 1577.244 & 0.000 & 3389.73 & 2985.28 & 1443.86 \\
p-value for k-1 & 0.000 & 0.2976 & 0.5828 & 0.6743 & 0.5962 & 0.6023 & 0.4133 & 0.6842 \\
Entropy & 0.896 & 0.853 & 0.857 & 0.875 & 0.916 & 0.881 & 0.872 & 0.839 \\
\hline
\end{tabular}

Bayesian Information Criterion BIC

Sample size Ajusted BIC, ABIC 
Table 5. Results from latent class analysis of health risk behaviors among adolescents

\begin{tabular}{|c|c|c|c|c|c|}
\hline \multicolumn{6}{|c|}{ Probability of risky behaviors in members of each class } \\
\hline & \multicolumn{5}{|c|}{ All adolescents $(\mathrm{N}=13,583)$} \\
\hline & $\begin{array}{l}\text { Class } 1 \\
\text { Low risk } \\
(52.6 \%)\end{array}$ & $\begin{array}{l}\text { Class } 2 \\
(14.9 \%)\end{array}$ & $\begin{array}{c}\text { Class } 3 \\
\text { Depressed and } \\
\text { Suicidal } \\
(14.4 \%)\end{array}$ & $\begin{array}{c}\text { Class } 4 \\
\text { Tobacco and } \\
\text { Alcohol } \\
\text { Users } \\
(9.4 \%) \\
\end{array}$ & $\begin{array}{c}\text { Class } 5 \\
\text { Polysubstance } \\
\text { Users } \\
(8.5 \%)\end{array}$ \\
\hline Current Cigarette Use & 0.000 & 0.000 & 0.004 & 0.971 & 0.791 \\
\hline Daily Cigarette Smoker & 0.002 & 0.031 & 0.028 & 0.353 & 0.523 \\
\hline 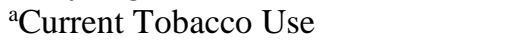 & 0.025 & 0.248 & 0.044 & 0.989 & 0.898 \\
\hline Current Marijuana Use & 0.041 & 0.452 & 0.169 & 0.522 & 0.818 \\
\hline Current Alcohol Use & 0.062 & 0.866 & 0.292 & 0.735 & 0.933 \\
\hline bBinge Drinker & 0.000 & 0.560 & 0.096 & 0.506 & 0.794 \\
\hline \multicolumn{6}{|l|}{$\begin{array}{l}\text { Lifetime Drug Use (past } 12 \\
\text { months) }\end{array}$} \\
\hline Cocaine & 0.001 & 0.051 & 0.023 & 0.046 & 0.453 \\
\hline Glue/Aerosol Spray/Inhalant & 0.018 & 0.075 & 0.148 & 0.089 & 0.443 \\
\hline Ecstasy & 0.003 & 0.078 & 0.023 & 0.070 & 0.491 \\
\hline 'Prescription Drugs & 0.027 & 0.250 & 0.184 & 0.326 & 0.774 \\
\hline \multicolumn{6}{|l|}{ Unintentional Injury } \\
\hline $\begin{array}{l}\text { Never or rarely wore bicycle } \\
\text { helmet }\end{array}$ & 0.833 & 0.935 & 0.902 & 0.936 & 0.939 \\
\hline Never or rarely wore seat belt & 0.039 & 0.092 & 0.055 & 0.135 & 0.244 \\
\hline $\begin{array}{l}\text { Rode with a driver who had } \\
\text { been drinking alcohol }\end{array}$ & 0.086 & 0.391 & 0.209 & 0.323 & 0.608 \\
\hline Drove when drinking alcohol & 0.000 & 0.181 & 0.019 & 0.157 & 0.476 \\
\hline $\begin{array}{l}\text { Texted while driving a car or } \\
\text { other vehicle }\end{array}$ & 0.266 & 0.634 & 0.341 & 0.512 & 0.690 \\
\hline \multicolumn{6}{|l|}{ Violence } \\
\hline Carried a weapon & 0.094 & 0.227 & 0.183 & 0.293 & 0.491 \\
\hline $\begin{array}{l}\text { Carried a weapon on school } \\
\text { property }\end{array}$ & 0.017 & 0.050 & 0.058 & 0.376 & 0.229 \\
\hline Were in a physical fight & 0.113 & 0.344 & 0.291 & 0.087 & 0.671 \\
\hline $\begin{array}{l}\text { dDepressive Symptoms (past } 12 \\
\text { months) }\end{array}$ & 0.120 & 0.208 & 0.812 & 0.308 & 0.636 \\
\hline Suicidal thoughts (past 12 months) & 0.018 & 0.031 & 0.645 & 0.147 & 0.533 \\
\hline $\begin{array}{l}\text { Plan to Attempt Suicide (past } 12 \\
\text { months) }\end{array}$ & 0.015 & 0.015 & 0.506 & 0.105 & 0.469 \\
\hline Bullied & 0.124 & 0.110 & 0.477 & 0.162 & 0.335 \\
\hline Electronically Bullied & 0.061 & 0.126 & 0.364 & 0.129 & 0.352 \\
\hline \multicolumn{6}{|l|}{ Risky Sexual Behavior } \\
\hline $\begin{array}{l}\text { Had sexual intercourse with } 4+ \\
\text { persons (during their life) }\end{array}$ & 0.041 & 0.281 & 0.084 & 0.241 & 0.602 \\
\hline $\begin{array}{l}\text { Were currently sexually active } \\
\text { (sexual intercourse with at least } \\
\text { one person during the } 3 \text { months } \\
\text { before the survey) }\end{array}$ & 0.160 & 0.607 & 0.269 & 0.560 & 0.833 \\
\hline Did not use condom & 0.325 & 0.373 & 0.446 & 0.376 & 0.563 \\
\hline $\begin{array}{l}\text { Drank alcohol or used drugs } \\
\text { before last sexual intercourse }\end{array}$ & 0.004 & 0.252 & 0.069 & 0.196 & 0.561 \\
\hline Unhealthy Weight Control & & & & & \\
\hline $\begin{array}{l}\text { Did not eat for } 24+\text { hours to } \\
\text { lose weight or keep from } \\
\text { gaining weight }\end{array}$ & 0.040 & 0.083 & 0.348 & 0.133 & 0.378 \\
\hline
\end{tabular}


Took diet pills, powders, or liquids

$\begin{array}{lllll}0.013 & 0.045 & 0.102 & 0.046 & 0.202 \\ & & & & 0.195 \\ 0.007 & 0.022 & 0.121 & 0.023 & \\ & & & & \\ 0.052 & 0.024 & 0.070 & 0.046 & 0.057 \\ 0.068 & 0.043 & 0.094 & 0.055 & 0.058 \\ 0.175 & 0.190 & 0.256 & 0.181 & 0.220 \\ 0.110 & 0.095 & 0.205 & 0.154 & 0.236 \\ 0.147 & 0.196 & 0.197 & 0.273 & 0.377 \\ 0.144 & 0.121 & 0.218 & 0.146 & 0.145 \\ 0.316 & 0.313 & 0.349 & 0.320 & 0.367 \\ 0.390 & 0.358 & 0.542 & 0.372 & 0.470\end{array}$

Vomited or took laxatives to

lose weight or keep from

gaining weight

Poor Dietary Behavior

No fruit

No vegetable

0.068

0.043

0.094

0.058

No milk

0.175

0.190

0.220

No breakfast

0.110

0.236

Drank soda 2+ times/day

eNo Physical Activity

Sedentary Behavior

Watched TV 3+ hours/day

Played Video Games 3+ hours/day

0.390

0.358

0.542

0.372

0.470

${ }^{\text {fWeight Status }}$

Overweight

$\begin{array}{ll}0.165 & 0.156\end{array}$

0.179

0.164

0.166

Obese

0.128

0.093

0.165

0.180

0.169

a Percentage of students who smoked cigarettes or cigars or used chewing tobacco, snuff, or dip on one or more of the past 30 days

b Percentage of students who had five or more drinks of alcohol in a row, that is, within a couple of hours, on one or more of the past 30 days

c Percentage of students who have taken a prescription drug (such as OxyContin, Percocet, Vicodin, Codeine, Adderall, Ritalin, or Xanax) without a doctor's prescription one or more times during their life

d Percentage of students who felt sad or hopeless almost every day for two weeks in a row that they stopped doing some usual activities during the past 12 months

e Percentage of students who were physically active for a total of at least 60 minutes per day on 0 days of the past 7 days

f Overweight ( BMI $\geq 85-94$ th) and Obese ( $\geq 95$ th percentile) 
Table 6. Results from latent class analysis of health risk behaviors among boys and girls

\begin{tabular}{|c|c|c|c|c|c|c|c|}
\hline \multicolumn{8}{|c|}{ Probability of risky behaviors in members of each class } \\
\hline & \multicolumn{3}{|c|}{ Boys $(n=6950)$} & \multicolumn{4}{|c|}{ Girls $(n=6621)$} \\
\hline & $\begin{array}{l}\text { Class } 1 \\
\text { Low } \\
\text { Risk } \\
(61.7 \%)\end{array}$ & $\begin{array}{l}\text { Class } 2 \\
\text { Alcohol } \\
\text { Drinker } \\
\quad \mathrm{s} \\
(26.1 \%)\end{array}$ & $\begin{array}{c}\text { Class } 3 \\
\text { Polysubstance } \\
\text { Users } \\
(12.2 \%)\end{array}$ & $\begin{array}{c}\text { Class } 1 \\
\text { Low Risk } \\
(49.9 \%)\end{array}$ & $\begin{array}{l}\text { Class } 2 \\
\text { Alcohol } \\
\text { Drinkers } \\
(19.8 \%)\end{array}$ & $\begin{array}{l}\text { Class } 3 \\
\text { Depressed } \\
\text { and Suicidal } \\
(18.7 \%)\end{array}$ & $\begin{array}{c}\text { Class } 4 \\
\text { Poly Substance } \\
\text { Users }- \text { High } \\
\text { Risk } \\
(11.6 \%)\end{array}$ \\
\hline Current Cigarette Use & 0.000 & 0.291 & 0.762 & 0.000 & 0.295 & 0.015 & 0.804 \\
\hline Daily Cigarette Smoker & 0.001 & 0.100 & 0.505 & 0.002 & 0.125 & 0.026 & 0.499 \\
\hline${ }^{\mathrm{a} C u r r e n t}$ Tobacco Use & 0.037 & 0.554 & 0.889 & 0.003 & 0.394 & 0.033 & 0.855 \\
\hline Current Marijuana Use & 0.055 & 0.455 & 0.781 & 0.030 & 0.459 & 0.151 & 0.744 \\
\hline Current Alcohol Use & 0.057 & 0.775 & 0.923 & 0.083 & 0.811 & 0.308 & 0.885 \\
\hline${ }^{\mathrm{b}}$ Binge Drinker & 0.000 & 0.512 & 0.756 & 0.000 & 0.521 & 0.092 & 0.706 \\
\hline \multicolumn{8}{|l|}{$\begin{array}{l}\text { Lifetime Drug Use (past } 12 \\
\text { months) }\end{array}$} \\
\hline Cocaine & 0.002 & 0.046 & 0.425 & 0.001 & 0.034 & 0.022 & 0.287 \\
\hline Glue/Aerosol Spray/Inhalant & 0.024 & 0.074 & 0.357 & 0.019 & 0.074 & 0.167 & 0.386 \\
\hline Ecstasy & 0.003 & 0.069 & 0.445 & 0.003 & 0.059 & 0.016 & 0.338 \\
\hline${ }^{\mathrm{c}}$ Prescription Drugs & 0.034 & 0.279 & 0.712 & 0.031 & 0.226 & 0.176 & 0.679 \\
\hline \multicolumn{8}{|l|}{ Unintentional Injury } \\
\hline $\begin{array}{l}\text { Never or rarely wore bicycle } \\
\text { helmet }\end{array}$ & 0.849 & 0.929 & 0.963 & 0.821 & 0.947 & 0.897 & 0.908 \\
\hline Never or rarely wore seat belt & 0.047 & 0.104 & 0.283 & 0.032 & 0.082 & 0.046 & 0.176 \\
\hline $\begin{array}{l}\text { Rode with a driver who had } \\
\text { been drinking alcohol }\end{array}$ & 0.090 & 0.338 & 0.554 & 0.095 & 0.367 & 0.213 & 0.550 \\
\hline Drove when drinking alcohol & 0.000 & 0.167 & 0.453 & 0.000 & 0.148 & 0.014 & 0.344 \\
\hline $\begin{array}{l}\text { Texted while driving a car or } \\
\text { other vehicle }\end{array}$ & 0.257 & 0.573 & 0.652 & 0.286 & 0.610 & 0.300 & 0.656 \\
\hline \multicolumn{8}{|l|}{ Violence } \\
\hline Carried a weapon & 0.172 & 0.366 & 0.666 & 0.021 & 0.083 & 0.122 & 0.255 \\
\hline $\begin{array}{l}\text { Carried a weapon on school } \\
\text { property }\end{array}$ & 0.033 & 0.082 & 0.279 & 0.004 & 0.025 & 0.044 & 0.128 \\
\hline Were in a physical fight & 0.175 & 0.389 & 0.751 & 0.071 & 0.257 & 0.240 & 0.539 \\
\hline $\begin{array}{l}{ }^{\mathrm{d}} \text { Depressive Symptoms (past } 12 \\
\text { months) }\end{array}$ & 0.158 & 0.191 & 0.491 & 0.169 & 0.322 & 0.840 & 0.736 \\
\hline Suicidal thoughts (past 12 months) & 0.072 & 0.089 & 0.389 & 0.030 & 0.040 & 0.685 & 0.628 \\
\hline $\begin{array}{l}\text { Plan to Attempt Suicide (past } 12 \\
\text { months) }\end{array}$ & 0.067 & 0.073 & 0.342 & 0.017 & 0.012 & 0.516 & 0.530 \\
\hline Bullied & 0.151 & 0.125 & 0.245 & 0.143 & 0.148 & 0.476 & 0.411 \\
\hline Electronically Bullied & 0.059 & 0.098 & 0.190 & 0.099 & 0.184 & 0.379 & 0.461 \\
\hline \multicolumn{8}{|l|}{ Risky Sexual Behavior } \\
\hline $\begin{array}{l}\text { Had sexual intercourse with } 4+ \\
\text { persons (during their life) }\end{array}$ & 0.054 & 0.248 & 0.559 & 0.182 & 0.616 & 0.263 & 0.790 \\
\hline $\begin{array}{l}\text { Were currently sexually active } \\
\text { (sexual intercourse with at least } \\
\text { one person during the } 3 \text { months } \\
\text { before the survey) }\end{array}$ & 0.145 & 0.533 & 0.780 & 0.029 & 0.232 & 0.062 & 0.535 \\
\hline Did not use condom & 0.301 & 0.287 & 0.466 & 0.360 & 0.449 & 0.457 & 0.613 \\
\hline $\begin{array}{l}\text { Drank alcohol or used drugs } \\
\text { before last sexual intercourse }\end{array}$ & 0.000 & 0.227 & 0.551 & 0.008 & 0.197 & 0.064 & 0.444 \\
\hline
\end{tabular}


Did not eat for 24+ hours to lose weight or keep from gaining weight

Took diet pills, powders, or liquids

Vomited or took laxatives to lose weight or keep from gaining weight

Poor Dietary Behavior

No fruit

$\begin{array}{lllllll}0.047 & 0.052 & 0.255 & 0.055 & 0.156 & 0.416 & 0.447 \\ 0.016 & 0.031 & 0.131 & 0.016 & 0.067 & 0.108 & 0.219 \\ & & & & & \\ 0.010 & 0.006 & 0.111 & 0.10 & 0.041 & 0.144 & 0.227 \\ & & & & & & \\ 0.069 & 0.033 & 0.082 & 0.039 & 0.026 & 0.053 & 0.047 \\ 0.087 & 0.037 & 0.101 & 0.050 & 0.055 & 0.092 & 0.296 \\ 0.136 & 0.111 & 0.159 & 0.230 & 0.282 & 0.264 & 0.210 \\ 0.122 & 0.111 & 0.254 & 0.102 & 0.110 & 0.219 & 0.314 \\ 0.171 & 0.245 & 0.422 & 0.126 & 0.179 & 0.167 & 0.177 \\ 0.122 & 0.072 & 0.143 & 0.177 & 0.205 & 0.224 & \end{array}$

No vegetable

No milk

No breakfast

$0.315 \quad 0.314$

0.424

0.324

0.313

0.332

0.316

Played Video Games 3+ hours/day

$0.447 \quad 0.355$

0.451

0.350

0.377

0.524

0.484

${ }^{f}$ Weight Status

Overweight

$\begin{array}{lll}0.165 & 0.164 & 0.153 \\ 0.128 & 0.131 & 0.246\end{array}$

0.158
0.097

0.166

0.190

0.167

Obese

0.128

0.246

0.087

0.137

0.147

a Percentage of students who smoked cigarettes or cigars or used chewing tobacco, snuff, or dip on one or more of the past 30 days

b Percentage of students who had five or more drinks of alcohol in a row, that is, within a couple of hours, on one or more of the past 30 days

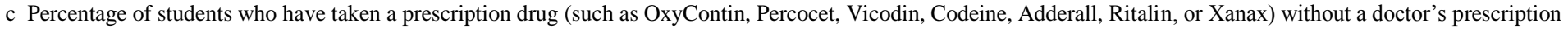
one or more times during their life

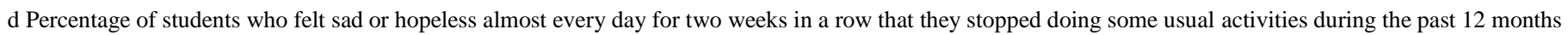
e Percentage of students who were physically active for a total of at least 60 minutes per day on 0 days of the past 7 days

f Overweight ( $\mathrm{BMI} \geq 85-94$ th) and Obese ( $\geq 95$ th percentile) 
Table 7. Results from latent class analysis of health risk behaviors among adolescents (3 Class Model)

\begin{tabular}{|c|c|c|c|}
\hline \multicolumn{4}{|c|}{ Probability of risky behaviors in members of each class } \\
\hline & \multicolumn{3}{|c|}{ All adolescents $(\mathrm{N}=13583)$} \\
\hline & $\begin{array}{l}\text { Class } 1 \\
(16.3 \%)\end{array}$ & $\begin{array}{l}\text { Class } 2 \\
(57.8 \%)\end{array}$ & $\begin{array}{l}\text { Class } 3 \\
(25.8 \%)\end{array}$ \\
\hline Current Cigarette Use & 0.015 & 0.014 & 0.589 \\
\hline Daily Cigarette Smoker & 0.022 & 0.005 & 0.319 \\
\hline${ }^{\mathrm{a} C u r r e n t ~ T o b a c c o ~ U s e ~}$ & 0.048 & 0.051 & 0.753 \\
\hline Current Marijuana Use & 0.153 & 0.072 & 0.657 \\
\hline Current Alcohol Use & 0.284 & 0.140 & 0.889 \\
\hline${ }^{\mathrm{b} B}$ Binge Drinker & 0.094 & 0.041 & 0.689 \\
\hline \multicolumn{4}{|l|}{$\begin{array}{l}\text { Lifetime Drug Use (past } 12 \\
\text { months) }\end{array}$} \\
\hline Cocaine & 0.018 & 0.002 & 0.197 \\
\hline Glue/Aerosol Spray/Inhalant & 0.137 & 0.021 & 0.215 \\
\hline Ecstasy & 0.017 & 0.005 & 0.233 \\
\hline${ }^{\mathrm{c}}$ Prescription Drugs & 0.165 & 0.037 & 0.502 \\
\hline \multicolumn{4}{|l|}{ Unintentional Injury } \\
\hline $\begin{array}{l}\text { Never or rarely wore bicycle } \\
\text { helmet }\end{array}$ & 0.898 & 0.843 & 0.944 \\
\hline Never or rarely wore seat belt & 0.052 & 0.042 & 0.168 \\
\hline $\begin{array}{l}\text { Rode with a driver who had } \\
\text { been drinking alcohol }\end{array}$ & 0.203 & 0.108 & \\
\hline Drove when drinking alcohol & 0.012 & 0.006 & 0.477 \\
\hline $\begin{array}{l}\text { Texted while driving a car or } \\
\text { other vehicle }\end{array}$ & 0.329 & 0.310 & 0.639 \\
\hline \multicolumn{4}{|l|}{ Violence } \\
\hline Carried a weapon & 0.168 & 0.105 & 0.357 \\
\hline $\begin{array}{l}\text { Carried a weapon on school } \\
\text { property }\end{array}$ & 0.051 & 0.020 & 0.128 \\
\hline Were in a physical fight & 0.280 & 0.128 & 0.496 \\
\hline $\begin{array}{l}{ }^{\mathrm{d} D e p r e s s i v e ~ S y m p t o m s ~(p a s t ~} 12 \\
\text { months) }\end{array}$ & 0.791 & 0.111 & 0.410 \\
\hline Suicidal thoughts (past 12 months) & 0.593 & 0.011 & 0.259 \\
\hline $\begin{array}{l}\text { Plan to Attempt Suicide (past } 12 \\
\text { months) }\end{array}$ & 0.458 & 0.010 & 0.214 \\
\hline Bullied & 0.462 & 0.113 & 0.215 \\
\hline Electronically Bullied & 0.352 & 0.060 & 0.216 \\
\hline \multicolumn{4}{|l|}{ Risky Sexual Behavior } \\
\hline $\begin{array}{l}\text { Had sexual intercourse with } 4+ \\
\text { persons (during their life) }\end{array}$ & 0.069 & 0.058 & 0.411 \\
\hline $\begin{array}{l}\text { Were currently sexually active } \\
\text { (sexual intercourse with at least } \\
\text { one person during the } 3 \text { months } \\
\text { before the survey) }\end{array}$ & 0.257 & 0.199 & 0.711 \\
\hline Did not use condom & 0.554 & 0.316 & 0.459 \\
\hline $\begin{array}{l}\text { Drank alcohol or used drugs } \\
\text { before last sexual intercourse }\end{array}$ & 0.051 & 0.030 & 0.387 \\
\hline Unhealthy Weight Control & & & \\
\hline $\begin{array}{l}\text { Did not eat for } 24+\text { hours to } \\
\text { lose weight or keep from } \\
\text { gaining weight }\end{array}$ & 0.330 & 0.039 & 0.210 \\
\hline
\end{tabular}


Took diet pills, powders, or liquids

$\begin{array}{lll}0.094 & 0.015 & 0.104\end{array}$

Vomited or took laxatives to

lose weight or keep from

$\begin{array}{lll}0.110 & 0.006 & 0.087\end{array}$

gaining weight

Poor Dietary Behavior

No fruit

No vegetable

$\begin{array}{lll}0.067 & 0.047 & 0.048\end{array}$

No milk

$\begin{array}{lll}0.088 & 0.065 & 0.055\end{array}$

No breakfast

0.250

$0.174 \quad 0.201$

Drank soda $2+$ times/day

0.197

$0.107 \quad 0.167$

No Physical Activity

0.192

0.149

0.295

Sedentary Behavior

Watched TV 3+ hours/day

0.216

0.138

0.142

Played Video Games 3+ hours/day

$\begin{array}{lll}0.353 & 0.316 & 0.329\end{array}$

$\begin{array}{lll}0.541 & 0.383 & 0.399\end{array}$

${ }^{\text {fWeight Status }}$

\begin{tabular}{|c|c|}
\hline Overweight & 0.176 \\
\hline
\end{tabular}

Obese $\quad 0.164 \quad 0.125 \quad 0.145$

a Percentage of students who smoked cigarettes or cigars or used chewing tobacco, snuff, or dip on one or

more of the past 30 days

b Percentage of students who had five or more drinks of alcohol in a row, that is, within a couple of hours,

on one or more of the past 30 days

c Percentage of students who have taken a prescription drug (such as OxyContin, Percocet, Vicodin,

Codeine, Adderall, Ritalin, or Xanax) without a doctor's prescription one or more times during their life

d Percentage of students who felt sad or hopeless almost every day for two weeks in a row that they stopped

doing some usual activities during the past 12 months

e Percentage of students who were physically active for a total of at least 60 minutes per day on 0 days of

the past 7 days

f Overweight ( $\mathrm{BMI} \geq 85-94$ th) and Obese $(\geq 95$ th percentile) 


\section{Chapter 4}

Table 1. Demographic characteristics of adolescents in the 2013 national YRBSS $(\mathrm{N}=13,583)$.

\begin{tabular}{|c|c|c|}
\hline & Frequency & Weighted $\%$ \\
\hline \multicolumn{3}{|l|}{ Age } \\
\hline 12 years or younger & 26 & 0.2 \\
\hline 13 years old & 18 & 0.1 \\
\hline 14 years old & 1,368 & 10.0 \\
\hline 15 years old & 3,098 & 24.1 \\
\hline 16 years old & 3,203 & 25.3 \\
\hline 17 years old & 3,473 & 24.6 \\
\hline 18 years old & 2,320 & 15.6 \\
\hline \multicolumn{3}{|l|}{ Sex } \\
\hline Female & 6,621 & 50.0 \\
\hline Male & 6,950 & 50.0 \\
\hline \multicolumn{3}{|l|}{ Race/Ethnicity } \\
\hline American Indian/Alaska Native & 121 & 0.7 \\
\hline Asian & 491 & 3.0 \\
\hline Black or African American & 2,993 & 14.3 \\
\hline Native Hawaiian/Other PI & 135 & 0.8 \\
\hline White & 5,449 & 55.6 \\
\hline Hispanic/Latino & 1,734 & 10.4 \\
\hline Multiple - Hispanic & 1,661 & 10.7 \\
\hline Multiple - non-Hispanic & 681 & 4.4 \\
\hline \multicolumn{3}{|l|}{ Grade } \\
\hline $9^{\text {th }}$ & 3,588 & 27.3 \\
\hline $10^{\text {th }}$ & 3,152 & 25.7 \\
\hline $11^{\text {th }}$ & 3,184 & 23.8 \\
\hline $12^{\text {th }}$ & 3,557 & 23.1 \\
\hline
\end{tabular}


Table 2. Behavioral characteristics of adolescents in the 2013 national YRBSS

\begin{tabular}{|c|c|c|c|}
\hline & $\begin{array}{l}\text { Total Sample } \\
(\mathrm{n}=13,571)\end{array}$ & $\begin{array}{l}\text { Female } \\
(\mathrm{n}=6621)\end{array}$ & $\begin{array}{l}\text { Male } \\
(\mathrm{N}=6950)\end{array}$ \\
\hline Behavioral characteristics & $\mathrm{n}(\%)$ & $\mathrm{n}(\%)$ & $\mathrm{n}(\%)$ \\
\hline \multicolumn{4}{|l|}{ Tobacco Use } \\
\hline $\begin{array}{l}\text { Currently smoked cigarettes ( } 1 \text { or more days in the } \\
\text { past } 30 \text { days) }\end{array}$ & $1886(15.1)$ & $807(14.5)$ & $1079(15.6)$ \\
\hline $\begin{array}{l}\text { Smoked daily at least } 1 \text { cigarette every day/past } 30 \\
\text { days }\end{array}$ & $1039(8.3)$ & $430(8.2)$ & $609(8.4)$ \\
\hline $\begin{array}{l}\text { Currently used tobacco (cigarette use, current } \\
\text { smokeless tobacco use, or current cigar use) }\end{array}$ & $2666(21.1)$ & $997(17.0)$ & $1669(25.2)$ \\
\hline \multicolumn{4}{|l|}{ Marijuana use } \\
\hline $\begin{array}{l}\text { Currently used marijuana ( } 1+\text { days during the last } 30 \\
\text { days) }\end{array}$ & $3364(23.0)$ & $1498(21.6)$ & $1866(24.4)$ \\
\hline \multicolumn{4}{|l|}{ Alcohol use } \\
\hline $\begin{array}{l}\text { Currently drank alcohol (1+ days during the last } 30 \\
\text { days) }\end{array}$ & $4369(32.0)$ & $2180(32.6)$ & $2189(31.3)$ \\
\hline $\begin{array}{l}5+\text { Drinks in a row/one or more times in the past } 30 \\
\text { days }\end{array}$ & $2620(20.1)$ & $1189(19.1)$ & $1425(21.0)$ \\
\hline \multicolumn{4}{|l|}{ Other Drug Use (lifetime use) } \\
\hline cocaine & $746(5.5)$ & $271(4.4)$ & $475(6.5)$ \\
\hline $\begin{array}{l}\text { Sniffed glue, breathed content of aerosol spray cans, } \\
\text { inhaled paints or sprays to get high }\end{array}$ & $1175(8.8)$ & $622(9.8)$ & $553(7.7)$ \\
\hline Ecstasy & $935(6.4)$ & $368(5.5)$ & $567(7.4)$ \\
\hline *Prescription drug without a doctor's prescription & $2347(17.6)$ & $1069(17.1)$ & $1278(18.1)$ \\
\hline \multicolumn{4}{|l|}{ Unintentional injuries } \\
\hline Never or rarely wore bicycle helmet & $7687(57.7)$ & $3256(51.2)$ & $4431(64.1)$ \\
\hline Never or rarely wore seat belt & $1051(7.6)$ & $405(6.1)$ & $646(9.1)$ \\
\hline Rode with a driver who had been drinking alcohol & $3072(21.8)$ & $1538(22.3)$ & $1534(21.4)$ \\
\hline Drove when drinking alcohol & $759(5.9)$ & $253(4.4)$ & $506(7.5)$ \\
\hline Texted while driving a car or other vehicle & $3600(26.8)$ & $1422(23.6)$ & $1800(26.5)$ \\
\hline \multicolumn{4}{|l|}{ Violence } \\
\hline Carried a weapon & $2257(17.5)$ & $479(7.7)$ & $1778(27.2)$ \\
\hline Carried a weapon on school property & $627(5.2)$ & & \\
\hline Were in a physical fight & $3617(24.2)$ & 1385 (18.9) & $2232(29.5)$ \\
\hline \multicolumn{4}{|l|}{ Bullying } \\
\hline Were bullied on school property & $2505(19.5)$ & $1472(23.6)$ & $1033(15.4)$ \\
\hline Were electronically bullied & $1878(14.7)$ & $1300(20.9)$ & $578(8.5)$ \\
\hline \multicolumn{4}{|l|}{ Sadness/hopelessness } \\
\hline $\begin{array}{l}\text { Felt sad or hopeless (almost every day for } 2 \text { or more } \\
\text { weeks in a row in past year) }\end{array}$ & $4084(29.7)$ & $2592(38.9)$ & $1492(20.6)$ \\
\hline \multicolumn{4}{|l|}{ Suicidal ideation } \\
\hline $\begin{array}{l}\text { Seriously considered attempting suicide during the } \\
\text { past year }\end{array}$ & $2259(17.0)$ & $1469(22.2)$ & 789 (11.5) \\
\hline Made a plan about how they would attempt suicide & $1874(13.5)$ & $1175(16.8)$ & $699(10.2)$ \\
\hline \multicolumn{4}{|l|}{ Risky sexual behavior } \\
\hline $\begin{array}{l}\text { Had sexual intercourse with } 4+\text { persons (during their } \\
\text { life) }\end{array}$ & $2155(14.3)$ & $806(12.8)$ & $1349(15.7)$ \\
\hline
\end{tabular}


Were currently sexually active (sexual intercourse with at least one person during the 3 months before the survey)

Did not use condom

Drank alcohol or used drugs before last sexual intercourse

Weight Status

Were overweight $\left(\geq 85^{\text {th }}\right.$ percentile of BMI)

Were obese $\left(\geq 95^{\text {th }}\right.$ percentile of BMI)

Unhealthy Weight Control

Did not eat for 24+ hours to lose weight or keep from gaining weight

\begin{tabular}{|c|c|c|c|}
\hline nths before & $4663(32.4)$ & $2288(34.2)$ & $2375(30.7)$ \\
\hline & 1859 (12.9) & 1061 (17.9) & $798(10.2)$ \\
\hline & $1039(7.2)$ & $427(6.5)$ & $612(7.9)$ \\
\hline II) & 2064 (16.6) & $1044(16.6)$ & $1020(16.5)$ \\
\hline & $1786(13.7)$ & $732(10.8)$ & $1054(16.5)$ \\
\hline or keep from & 1781 (12.9) & $1218(18.5)$ & $563(7.3)$ \\
\hline & $684(4.9)$ & $431(6.5)$ & $253(3.3)$ \\
\hline or keep & $558(4.3)$ & $402(6.4)$ & $156(2.1)$ \\
\hline ces (during & $686(4.9)$ & 288 (3.9) & $398(5.9)$ \\
\hline 7 days before & $941(6.5)$ & $401(5.6)$ & $540(7.3)$ \\
\hline ys before the & $2816(19.0)$ & $1816(25.1)$ & $1000(12.9)$ \\
\hline times per day & 2588 (19.3) & $1091(16.3)$ & $1493(21.7)$ \\
\hline ays before & $1942(13.4)$ & 976 (13.6) & $966(13.2)$ \\
\hline & $2171(14.9)$ & $1383(18.9)$ & 788 (10.9) \\
\hline & 4777 (31.8) & $2320(31.8)$ & 2457 (31.9) \\
\hline ore hours/day & $5665(40.5)$ & $2742(39.8)$ & $2923(41.2)$ \\
\hline
\end{tabular}

Took diet pills, powders, or liquids

Vomited or took laxatives to lose weight or keep

from gaining weight

Poor dietary behavior

Did not eat fruit or drink 100\% fruit juices (during

the past 7 days before the survey)

Did not any vegetables (during the past 7 days before

the survey)

Did not drink milk (during the past 7 days before the survey)

Drank a can, bottle, or glass of soda $2+$ times per day

(during the past 7 days before the survey)

Did not eat breakfast (during the past 7 days before

the survey)

Physical inactivity and Sedentary Behavior

Physically active on 0 days past 7 days

Watched TV 3 or more hours/day
Played video or computer games 3 or more hours/day

weighted percentages are reported for representativeness of data to the US high school students

*prescription drugs such as OxyContin, Percocet, Vicodin, Codeine, Adderall, Ritalin, or Xanax

Row percentages reported for female and male students 
Table 3. OR and AOR for substance use among Overweight ( BMI $\left.\geq 85-94^{\text {th }}\right)$ and Obese $\left(\geq 95^{\text {th }}\right.$ percentile) High School Adolescents (2013 YRBSS)

\begin{tabular}{|c|c|c|c|c|c|c|c|c|}
\hline \multirow[b]{2}{*}{ Variables } & \multicolumn{2}{|c|}{ Cigarette Use } & \multicolumn{2}{|c|}{ Marijuana Use } & \multicolumn{2}{|c|}{ Alcohol Use } & \multicolumn{2}{|c|}{ Drug Use } \\
\hline & $\begin{array}{c}\text { OR } \\
(95 \% \mathrm{CI})\end{array}$ & $\begin{array}{c}\text { AOR } \\
(95 \% \mathrm{CI})\end{array}$ & $\begin{array}{c}\text { OR } \\
(95 \% \mathrm{CI})\end{array}$ & $\begin{array}{c}\text { AOR } \\
(95 \% \mathrm{CI})\end{array}$ & $\begin{array}{c}\text { OR } \\
(95 \% \mathrm{CI})\end{array}$ & $\begin{array}{c}\text { AOR } \\
(95 \% \mathrm{CI})\end{array}$ & $\begin{array}{c}\text { OR } \\
(95 \% \mathrm{CI})\end{array}$ & $\begin{array}{c}\text { AOR } \\
(95 \% \mathrm{CI})\end{array}$ \\
\hline \multicolumn{9}{|l|}{ Overweight } \\
\hline Yes & $\begin{array}{c}0.99 \\
(0.83-1.17)\end{array}$ & $\begin{array}{c}0.96 \\
(0.81-1.13)\end{array}$ & $\begin{array}{c}0.95 \\
(0.82-1.11)\end{array}$ & $\begin{array}{c}0.94 \\
(0.80-1.10)\end{array}$ & $\begin{array}{c}0.92 \\
(0.82-1.04)\end{array}$ & $\begin{array}{c}0.93 \\
(0.83-1.05)\end{array}$ & $\begin{array}{c}0.91 \\
(0.82-1.04)\end{array}$ & $\begin{array}{c}0.92 \\
(0.80-1.05)\end{array}$ \\
\hline No & $\begin{array}{c}1.00 \\
\text { (Referent) }\end{array}$ & $\begin{array}{c}1.00 \\
\text { (Referent) }\end{array}$ & $\begin{array}{c}1.00 \\
\text { (Referent) }\end{array}$ & $\begin{array}{c}1.00 \\
\text { (Referent) }\end{array}$ & $\begin{array}{c}1.00 \\
\text { (Referent) }\end{array}$ & $\begin{array}{c}1.00 \\
\text { (Referent) }\end{array}$ & $\begin{array}{c}1.00 \\
\text { (Referent) }\end{array}$ & $\begin{array}{c}1.00 \\
\text { (Referent) }\end{array}$ \\
\hline \multicolumn{9}{|l|}{ Obese } \\
\hline Yes & $\begin{array}{c}1.41 \\
(1.13-1.76)\end{array}$ & $\begin{array}{c}1.44 \\
(1.15-1.79)\end{array}$ & $\begin{array}{c}0.98 \\
(0.83-1.16)\end{array}$ & $\begin{array}{c}0.96 \\
(0.81-1.14)\end{array}$ & $\begin{array}{c}0.96 \\
(0.84-1.10)\end{array}$ & $\begin{array}{c}0.95 \\
(0.84-1.07)\end{array}$ & $\begin{array}{c}0.96 \\
(0.84-1.10)\end{array}$ & $\begin{array}{c}0.98 \\
(0.83-1.16)\end{array}$ \\
\hline No & $\begin{array}{c}1.00 \\
\text { (Referent) }\end{array}$ & $\begin{array}{c}1.00 \\
\text { (Referent) }\end{array}$ & $\begin{array}{c}1.00 \\
\text { (Referent) }\end{array}$ & $\begin{array}{c}1.00 \\
\text { (Referent) }\end{array}$ & $\begin{array}{c}1.00 \\
\text { (Referent) }\end{array}$ & $\begin{array}{c}1.00 \\
\text { (Referent) }\end{array}$ & $\begin{array}{c}1.00 \\
\text { (Referent) }\end{array}$ & $\begin{array}{c}1.00 \\
\text { (Referent) }\end{array}$ \\
\hline
\end{tabular}

Table 4. OR and AOR for Unintentional Injury, Violence, and Bullying among Overweight $\left(\mathrm{BMI} \geq 85-94^{\text {th }}\right.$ percentile) and Obese $\left(\geq 95^{\text {th }}\right.$ percentile) High School Adolescents (2013 YRBSS)

\begin{tabular}{|c|c|c|c|c|c|c|c|c|}
\hline \multirow[b]{2}{*}{ Variables } & \multicolumn{2}{|c|}{ Unintentional Injury } & \multicolumn{2}{|c|}{ Violence } & \multicolumn{2}{|c|}{ Bullied } & \multicolumn{2}{|c|}{ Electronically Bullied } \\
\hline & $\begin{array}{c}\text { OR } \\
(95 \% \mathrm{CI})\end{array}$ & $\begin{array}{c}\text { AOR } \\
(95 \% \mathrm{CI})\end{array}$ & $\begin{array}{c}\text { OR } \\
(95 \% \mathrm{CI})\end{array}$ & $\begin{array}{c}\text { AOR } \\
(95 \% \mathrm{CI})\end{array}$ & $\begin{array}{c}\text { OR } \\
(95 \% \mathrm{CI})\end{array}$ & $\begin{array}{c}\text { AOR } \\
(95 \% \mathrm{CI})\end{array}$ & $\begin{array}{c}\text { OR } \\
(95 \% \mathrm{CI})\end{array}$ & $\begin{array}{c}\text { AOR } \\
(95 \% \mathrm{CI})\end{array}$ \\
\hline \multicolumn{9}{|l|}{ Overweight } \\
\hline Yes & $\begin{array}{c}0.89 \\
(0.74-1.07)\end{array}$ & $\begin{array}{c}0.90 \\
(0.74-1.09)\end{array}$ & $\begin{array}{c}1.85 \\
(0.96-1.23)\end{array}$ & $\begin{array}{c}1.08 \\
(0.96-1.22)\end{array}$ & $\begin{array}{c}0.89 \\
(0.83-1.18)\end{array}$ & $\begin{array}{c}0.98 \\
(0.81-1.19)\end{array}$ & $\begin{array}{c}0.89 \\
(0.73-1.09)\end{array}$ & $\begin{array}{c}0.92 \\
(0.75-1.14)\end{array}$ \\
\hline No & $\begin{array}{c}1.00 \\
\text { (Referent) }\end{array}$ & $\begin{array}{c}1.00 \\
\text { (Referent) }\end{array}$ & $\begin{array}{c}1.00 \\
\text { (Referent) }\end{array}$ & $\begin{array}{c}1.00 \\
\text { (Referent) }\end{array}$ & $\begin{array}{c}1.00 \\
\text { (Referent) }\end{array}$ & $\begin{array}{c}1.00 \\
\text { (Referent) }\end{array}$ & $\begin{array}{c}1.00 \\
\text { (Referent) }\end{array}$ & $\begin{array}{c}1.00 \\
\text { (Referent) }\end{array}$ \\
\hline \multicolumn{9}{|l|}{ Obese } \\
\hline Yes & $\begin{array}{c}0.89 \\
(0.77-1.02)\end{array}$ & $\begin{array}{c}0.83 \\
(0.73-0.95)\end{array}$ & $\begin{array}{c}1.54 \\
(1.36-1.74)\end{array}$ & $\begin{array}{c}1.39 \\
(1.24-1.57)\end{array}$ & $\begin{array}{c}1.31 \\
(1.15-1.49)\end{array}$ & $\begin{array}{c}1.47 \\
(1.28-1.68)\end{array}$ & $\begin{array}{c}1.00 \\
(0.84-1.19)\end{array}$ & $\begin{array}{c}1.20 \\
(1.003-1.447)\end{array}$ \\
\hline No & $\begin{array}{c}1.00 \\
\text { (Referent) }\end{array}$ & $\begin{array}{c}1.00 \\
\text { (Referent) }\end{array}$ & $\begin{array}{c}1.00 \\
\text { (Referent) }\end{array}$ & $\begin{array}{c}1.00 \\
\text { (Referent) }\end{array}$ & $\begin{array}{c}1.00 \\
\text { (Referent) }\end{array}$ & $\begin{array}{c}1.00 \\
\text { (Referent) }\end{array}$ & $\begin{array}{c}1.00 \\
\text { (Referent) }\end{array}$ & $\begin{array}{c}1.00 \\
\text { (Referent) }\end{array}$ \\
\hline
\end{tabular}


Table 5. OR and AOR for Indicators of Risky Sexual Behavior among Overweight (BMI $\geq 85-94^{\text {th }}$ percentile) and Obese ( $\geq 95^{\text {th }}$ percentile) High School Adolescents (2013 YRBSS)

\begin{tabular}{|c|c|c|c|c|c|c|c|c|}
\hline \multirow[b]{2}{*}{ Variables } & \multicolumn{2}{|c|}{ Depression } & \multicolumn{2}{|c|}{ Suicidality } & \multicolumn{2}{|c|}{ Suicide Plan } & \multicolumn{2}{|c|}{ Risky Sexual Behavior } \\
\hline & $\begin{array}{c}\text { OR } \\
(95 \% \mathrm{CI})\end{array}$ & $\begin{array}{c}\text { AOR } \\
(95 \% \mathrm{CI})\end{array}$ & $\begin{array}{c}\text { OR } \\
(95 \% \mathrm{CI})\end{array}$ & $\begin{array}{c}\text { AOR } \\
(95 \% \mathrm{CI})\end{array}$ & $\begin{array}{c}\text { OR } \\
(95 \% \mathrm{CI})\end{array}$ & $\begin{array}{c}\text { AOR } \\
(95 \% \mathrm{CI})\end{array}$ & $\begin{array}{c}\text { OR } \\
(95 \% \mathrm{CI})\end{array}$ & $\begin{array}{c}\text { AOR } \\
(95 \% \mathrm{CI})\end{array}$ \\
\hline \multicolumn{9}{|l|}{ Overweight } \\
\hline Yes & $\begin{array}{c}1.12 \\
(0.94-1.33)\end{array}$ & $\begin{array}{c}1.12 \\
(0.94-1.34)\end{array}$ & $\begin{array}{c}1.02 \\
(0.83-1.25)\end{array}$ & $\begin{array}{c}1.01 \\
(0.82-1.26)\end{array}$ & $\begin{array}{c}1.02 \\
(0.85-1.23)\end{array}$ & $\begin{array}{c}1.03 \\
(0.85-1.24)\end{array}$ & $\begin{array}{c}0.96 \\
(0.86-1.08)\end{array}$ & $\begin{array}{c}0.98 \\
(0.80-1.02)\end{array}$ \\
\hline No & $\begin{array}{c}1.00 \\
\text { (Referent) }\end{array}$ & $\begin{array}{c}1.00 \\
\text { (Referent) }\end{array}$ & $\begin{array}{c}1.00 \\
\text { (Referent) }\end{array}$ & $\begin{array}{c}1.00 \\
\text { (Referent) }\end{array}$ & $\begin{array}{c}1.00 \\
\text { (Referent) }\end{array}$ & $\begin{array}{c}1.00 \\
\text { (Referent) }\end{array}$ & $\begin{array}{c}1.00 \\
\text { (Referent) }\end{array}$ & $\begin{array}{c}1.00 \\
\text { (Referent) }\end{array}$ \\
\hline \multicolumn{9}{|l|}{ Obese } \\
\hline Yes & $\begin{array}{c}1.07 \\
(0.94-1.21)\end{array}$ & $\begin{array}{c}1.19 \\
(1.04-1.37)\end{array}$ & $\begin{array}{c}1.34 \\
(1.17-1.54)\end{array}$ & $\begin{array}{c}1.51 \\
(1.31-1.76)\end{array}$ & $\begin{array}{c}1.46 \\
(1.24-1.73)\end{array}$ & $\begin{array}{c}1.61 \\
(1.35-1.92)\end{array}$ & $\begin{array}{c}0.91 \\
(0.80-1.02)\end{array}$ & $\begin{array}{c}0.86 \\
(0.75-0.98)\end{array}$ \\
\hline No & $\begin{array}{c}1.00 \\
\text { (Referent) }\end{array}$ & $\begin{array}{c}1.00 \\
\text { (Referent) }\end{array}$ & $\begin{array}{c}1.00 \\
\text { (Referent) }\end{array}$ & $\begin{array}{c}1.00 \\
\text { (Referent) }\end{array}$ & $\begin{array}{c}1.00 \\
\text { (Referent) }\end{array}$ & $\begin{array}{c}1.00 \\
\text { (Referent) }\end{array}$ & $\begin{array}{c}1.00 \\
\text { (Referent) }\end{array}$ & $\begin{array}{c}1.00 \\
\text { (Referent) }\end{array}$ \\
\hline
\end{tabular}

Table 6. OR and AOR for Unhealthy Weight Control, Poor Dietary Habits, and Sedentary Behavior among Overweight (BMI $\geq 85-94^{\text {th }}$ percentile) and Obese $\left(\geq 95^{\text {th }}\right.$ percentile) High School Adolescents (2013 YRBSS)

\begin{tabular}{ccccccc}
\hline \multirow{2}{*}{ Variables } & \multicolumn{2}{c}{ Unhealthy Weight Control } & \multicolumn{2}{c}{ Poor Dietary Habits } & \multicolumn{2}{c}{ Sedentary Behavior } \\
\cline { 2 - 6 } & OR & AOR & OR & AOR & OR & AOR \\
& $(95 \% \mathrm{CI})$ & $(95 \% \mathrm{CI})$ & $(95 \% \mathrm{CI})$ & $(95 \% \mathrm{CI})$ & $(95 \% \mathrm{CI})$ & $(95 \% \mathrm{CI})$ \\
\hline Overweight & & & & & & \\
Yes & 2.99 & 3.26 & 1.04 & 1.02 & 1.03 & 1.00 \\
& $(2.53-3.55)$ & $(2.77-3.84)$ & $(0.94-1.15)$ & $(0.92-1.13)$ & $(0.92-1.16)$ & $(0.89-1.12)$ \\
No & 1.00 & 1.00 & 1.00 & 1.00 & 1.00 & 1.00 \\
Obese & (Referent) & (Referent) & (Referent) & (Referent) & (Referent) & (Referent) \\
Yes & & & & & \\
& & & & 1.37 & 1.72 & 1.74 \\
No & $(4.53-6.60)$ & $(6.33-9.04)$ & $(1.18-1.54)$ & $(1.20-1.55)$ & $(1.20-1.55)$ & $(1.52-1.99)$ \\
& 1.00 & 1.00 & 1.00 & 1.00 & 1.00 & 1.00 \\
& (Referent) & (Referent) & (Referent) & (Referent) & (Referent) & (Referent) \\
\hline
\end{tabular}




\section{Chapter 5}

Table 1. Characteristics of high school adolescents in the U.S. $(\mathrm{N}=13,583)$

\begin{tabular}{|c|c|c|c|}
\hline & Frequency & Weighted \% & SE \\
\hline \multicolumn{4}{|l|}{ Age (years) } \\
\hline$\leq 14$ & 1412 & 10.4 & 0.53 \\
\hline 15 & 3098 & 24.1 & 0.61 \\
\hline 16 & 3203 & 25.3 & 0.63 \\
\hline 17 & 3473 & 24.6 & 0.54 \\
\hline$>=18$ & 2320 & 15.6 & 0.72 \\
\hline \multicolumn{4}{|l|}{ Grade } \\
\hline $9^{\text {th }}$ & 3588 & 27.3 & 0.60 \\
\hline $10^{\text {th }}$ & 3152 & 25.7 & 0.52 \\
\hline $11^{\text {th }}$ & 3184 & 23.9 & 0.48 \\
\hline $12^{\text {th }}$ & 3557 & 23.1 & 0.62 \\
\hline \multicolumn{4}{|l|}{ Race } \\
\hline White & 5449 & 55.6 & 3.60 \\
\hline Black & 2993 & 14.3 & 2.05 \\
\hline Hispanic & 1734 & 10.4 & 1.55 \\
\hline Hispanic multiple & 1661 & 10.7 & 0.89 \\
\hline Other* & 1428 & 8.9 & 0.92 \\
\hline \multicolumn{4}{|l|}{ Weight Status } \\
\hline Normal weight & 9537 & 69.7 & 0.88 \\
\hline Overweight & 2064 & 16.6 & 0.57 \\
\hline Obese & 1786 & 13.7 & 0.56 \\
\hline \multicolumn{4}{|l|}{ Depression } \\
\hline Yes & 4086 & 29.9 & 0.81 \\
\hline No & 9409 & 70.1 & \\
\hline \multicolumn{4}{|l|}{ Poor Dietary Habits } \\
\hline${ }^{\text {a} N o ~ v e g e t a b l e s ~}$ & 942 & 6.6 & 0.36 \\
\hline${ }^{b}$ No fruit & 687 & 5.0 & 0.31 \\
\hline${ }^{\mathrm{c} N o}$ milk & 2818 & 19.4 & 0.73 \\
\hline${ }^{\mathrm{d}}$ No breakfast & 1945 & 13.7 & 0.73 \\
\hline $\begin{array}{l}\text { eSoda intake } 2+\text { times per } \\
\text { day }\end{array}$ & 2588 & 19.3 & 1.49 \\
\hline \multicolumn{4}{|l|}{ Physical Inactivity } \\
\hline${ }^{\mathrm{f}}$ No physical activity & 2172 & 15.2 & 0.67 \\
\hline \multicolumn{4}{|l|}{ Sedentary Behavior } \\
\hline${ }^{9} 3+$ hours of $\mathrm{TV}$ & 4782 & 32.5 & 1.08 \\
\hline h $3+$ hours of video games & 5669 & 41.3 & 1.04 \\
\hline \multicolumn{4}{|l|}{$\dagger$ Tobacco Use } \\
\hline Yes & 2668 & 22.4 & 1.24 \\
\hline No & 10156 & 73.3 & 1.43 \\
\hline \multicolumn{4}{|c|}{$\begin{array}{l}\text { Weighted percentages were obtained to control for complex sample design, therefore division of individual cell sizes by the total sample will not } \\
\text { reflect weighted percentages. }\end{array}$} \\
\hline \multicolumn{4}{|c|}{ *Other includes Asian, American Indian/Alaska Native, Native Hawaiian/other PI, and multiple non-Hispanic } \\
\hline \multicolumn{4}{|c|}{ a Percentage of students who ate vegetables 0 times per day during the past seven days } \\
\hline b Percentage of students who ate fruits or drat & juices 0 times per & seven days & \\
\hline
\end{tabular}


c Percentage of students who drank 0 glasses per day of milk during the past seven days

d Percentage of students who ate breakfast on none of the past seven days

e Percentage of students who drank a can, bottle, or glass of soda or pop two or more times per day during the past seven days

f Percentage of students who were physically active for a total of at least 60 minutes per day on 0 of the past seven days

g Percentage of students who watched three or more hours per day of TV on an average school day

h Percentage of students who played video or computer games or used a computer for something that was not school work three or more hours per

day on an average school day

$\nmid$ Percentage of students who smoked cigarettes or cigars or used chewing tobacco, snuff, or dip on one or more of the past 30 days

Table 2. Crude odds ratio (OR) and adjusted odds ratio of tobacco use by poor dietary habits, physical inactivity, and sedentary behavior, and sociodemographic and health factors among female high school adolescents in the U.S.

(multiple logistic regression analysis).

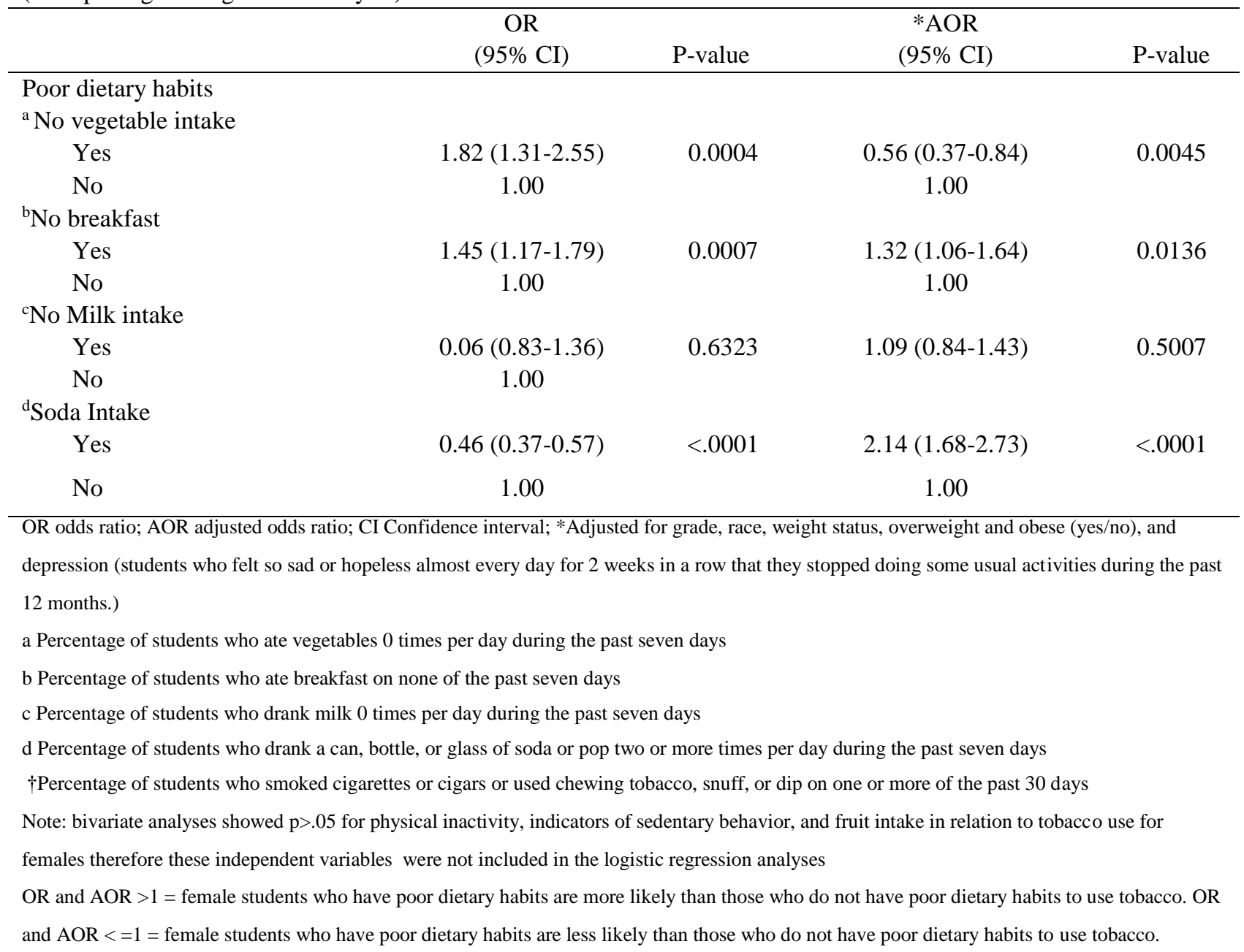


Table 3. Crude odds ratio (OR) and adjusted odds ratio of tobacco use by poor dietary habits, physical inactivity and sedentary behavior, and sociodemographic and health factors among male high school adolescents in the U.S. (multiple logistic regression analysis).

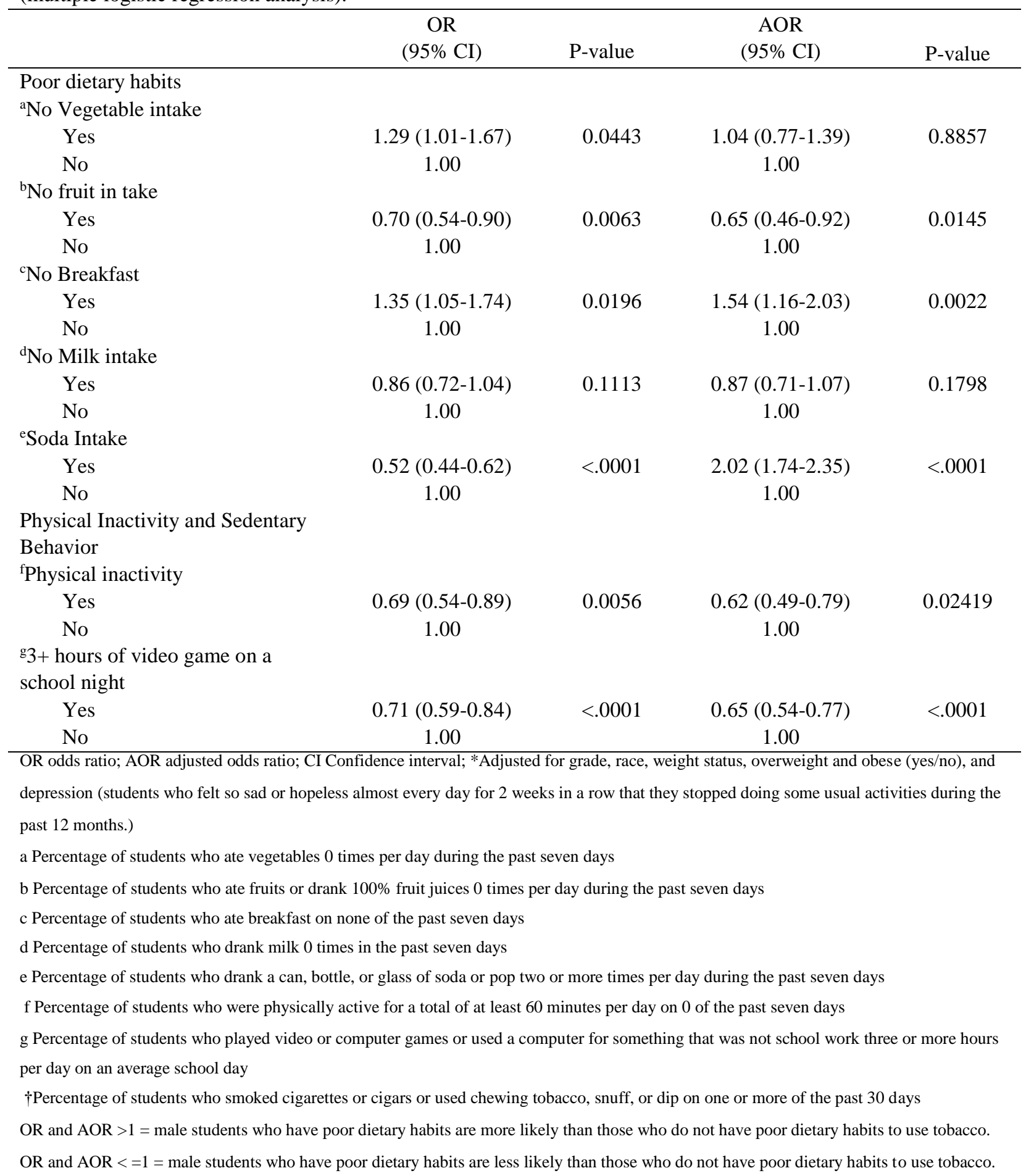

